TRANSACTIONS OF THE

AMERICAN MATHEMATICAL SOCIETY

Volume 355, Number 5, Pages 2011-2046

S 0002-9947(03)03208-2

Article electronically published on January 10, 2003

\title{
FUNCTORIAL HODGE IDENTITIES AND QUANTIZATION
}

\author{
M. J. SLUPINSKI
}

\begin{abstract}
By a uniform abstract procedure, we obtain integrated forms of the classical Hodge identities for Riemannian, Kähler and hyper-Kähler manifolds, as well as of the analogous identities for metrics of arbitrary signature. These identities depend only on the type of geometry and, for each of the three types of geometry, define a multiplicative functor from the corresponding category of real, graded, flat vector bundles to the category of infinite-dimensional $\mathbf{Z}_{2}$-projective representations of an algebraic structure. We define new multiplicative numerical invariants of closed Kähler and hyper-Kähler manifolds which are invariant under deformations of the metric.
\end{abstract}

\section{INTRODUCTION}

Let $M$ be an oriented manifold with non-degenerate metric of arbitrary signature. The fundamental operators acting in $\Gamma_{M}(\Lambda)$, the space of real differential forms over $M$, are the degree $\partial$, the Hodge star operator $*$, the exterior derivative $d$, its formal adjoint $d^{*}$ and the Hodge d'Alembertian $\square=d d^{*}+d^{*} d$. These operators satisfy curious algebraic relations, first proved for positive-definite metrics by Hodge $[\mathrm{H}$, Kodaira $[\mathrm{K}$ and Bidal-de Rham $\mathrm{BdR}$ in the early nineteen-forties. Hodge went on to prove similar relations for a larger family of operators defined on complex projective manifolds and from them he deduced many of his now classical results for these manifolds $[\mathrm{H}]$. A few years later, these relations were given their modern form and shown to be valid on arbitrary Kähler manifolds by Weil [W1] and, much more recently, a set of similar relations has been found between certain operators defined on hyper-Kähler manifolds ([FKS], [V], Wi]).

In this article we give a uniform, abstract derivation of new sets of relations for these three types of geometry, and show that the relations we obtain are functorial. The new relations imply the known relations but are more general in two ways: they describe Lie group rather than Lie algebra actions on differential operators and are valid in arbitrary signature. They are functorial in the sense that for each type of geometry, they define a multiplicative functor from the category of real, flat, graded vector bundles to the category of infinite-dimensional $\mathbf{Z}_{\mathbf{2}}$-projective representations of an algebraic structure. The point is that the algebraic structure depends only on the type of geometry and not on the manifold, metric or flat vector bundle.

Each of the three algebraic structures which emerges in this context is a pair $(G, \mathfrak{s})$, where $\mathfrak{s}$ is a real Lie superalgebra and $G$ is a group of automorphisms of $\mathfrak{s}$. Mathematically, this kind of structure is closely related to the notions of HarishChandra pair and Lie supergroup defined by Bernstein in [B]. In the language of

Received by the editors April 17, 2002 and, in revised form, July 2, 2002.

2000 Mathematics Subject Classification. Primary 22E99, 53C50, 53C55, 53C99. 
physics, representing such a pair can perhaps be thought of in terms of canonical quantization. In any case, a detailed understanding of the representation theory of the pairs $(G, \mathfrak{s})$ should have many applications in the theory of manifolds with the corresponding type of geometry as well as providing a unified framework in which to understand known results. As a step in this direction, we give a proof of the Hodge index theorem for closed Kähler manifolds which does not use the Hodge-Riemann bilinear relations, and we define families of new multiplicative numerical invariants for closed Kähler and hyper-Kähler manifolds.

We now give a more detailed account of the contents of this article. Let $T$ be an $n$-dimensional real vector space. By a $\mathbf{K}$-metric $g_{\mathbf{K}}$ on $T$ we will mean, respectively, a metric, a metric with compatible complex structure or a metric with compatible quaternionic structure on $T$, depending on whether $\mathbf{K}=\mathbf{R}, \mathbf{C}$ or $\mathbf{H}$. The group of automorphisms $O\left(T, g_{\mathbf{K}}\right)$ of a $\mathbf{K}$-metric $g_{\mathbf{K}}$ is embedded in $O\left(T \oplus T^{*}\right)$ (duality defines a canonical metric on $T \oplus T^{*}$ ) by the map $g \mapsto g \oplus g^{t^{-1}}$ and $O\left(T, g_{\mathbf{K}}\right)^{\prime}$, its commutant in $O\left(T \oplus T^{*}\right)$, acts naturally by composition on $I\left(T, g_{\mathbf{K}}\right)=H_{o m}\left(T, g_{\mathbf{K}}\right)\left(T^{*}, T \oplus T^{*}\right)$, the space of $O\left(T, g_{\mathbf{K}}\right)$-equivariant maps from $T^{*}$ to $T \oplus T^{*}$. Our first main theorem is that $O\left(T, g_{\mathbf{K}}\right)^{\prime}$ and $I\left(T, g_{\mathbf{K}}\right)$ are independent of $\left(T, g_{\mathbf{K}}\right)$. More precisely (cf. Section 3$)$ :

Theorem. $I\left(T, g_{\mathbf{K}}\right)$ is naturally a $\mathbf{K}$-bimodule, has a natural metric $\lambda_{I\left(T, g_{\mathbf{K}}\right)}$ and there is a canonical isometry of $\mathbf{K}$-bimodules $i_{T}: \mathbf{K}^{1,1} \rightarrow I\left(T, g_{\mathbf{K}}\right)$ inducing an

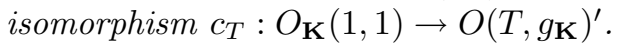

Here, $\mathbf{K}^{1,1}$ denotes $\mathbf{K}^{2}$ with the metric $\lambda\left((x, y),\left(x^{\prime}, y^{\prime}\right)\right)=\operatorname{Re}\left(\frac{1}{2}\left(\bar{x} y^{\prime}+\bar{y} x^{\prime}\right)\right)$ and $O_{\mathbf{K}}(1,1)$ is the group of right $\mathbf{K}$-linear isometries of $\lambda$. Next, we define $\operatorname{Gr}\left(T, g_{\mathbf{K}}\right)$ to be the inverse image of $O\left(T, g_{\mathbf{K}}\right)^{\prime}$ under the double covering map $\pi: \operatorname{Pin}\left(T \oplus T^{*}\right) \rightarrow O\left(T \oplus T^{*}\right)$. The map $c_{T}^{-1} \circ \pi: G r\left(T, g_{\mathbf{K}}\right) \rightarrow O_{\mathbf{K}}(1,1)$ is a double covering map and, together with the $\mathbf{Z}_{2}$-grading induced by inclusion in $C\left(T \oplus T^{*}\right)$, forms a graded double cover (GDC) of $O_{\mathbf{K}}(1,1)$ (cf. [S]). This GDC is not independent of $\left(T, g_{\mathbf{K}}\right)$. However, the derivative of $c_{T}^{-1} \circ \pi$ is an isomorphism of Lie algebras, so that $\operatorname{gr}\left(T, g_{\mathbf{K}}\right)$ is always canonically isomorphic to $o_{\mathbf{K}}(1,1)$. For example, if $\mathbf{K}=\mathbf{C}, o_{\mathbf{K}}(1,1)=u(1,1) \cong \mathbf{R} \oplus \operatorname{sl}(2, \mathbf{R})$ and if $\mathbf{K}=\mathbf{H}$, $o_{\mathbf{K}}(1,1)=s p(1,1) \cong s o(4,1)$. These isomorphisms, as we show, are ultimately the source of the $\operatorname{sl}(2, \mathbf{R})$ occurring in classical Hodge-Lefschetz theory (cf. W2]) and the $s o(5, \mathbf{C})$ introduced by Verbitsky in its analogue for hyper-Kähler manifolds (cf. $[\mathrm{V}])$. In the physics literature, these $\operatorname{sl}(2, \mathbf{R})$ and $s o(5, \mathbf{C})$ symmetries follow via "dimensional reduction" from the symmetries of certain supersymmetric sigma models (cf. [FKS], [Wi]).

In order to express the multiplicative properties of our constructions we introduce a product $\star$ in the category of GDCs of $O_{\mathbf{K}}(1,1)$ and a tensor product in the category of graded representations of GDCs of $O_{\mathbf{K}}(1,1)$ (cf. Section 2). The exterior algebra $\Lambda\left(T^{*}\right)$ is a graded representation of $\operatorname{Gr}\left(T, g_{\mathbf{K}}\right)$ and in these categories one has isomorphisms (cf. Section 4):

Theorem. $\operatorname{Gr}\left(T \oplus T^{\prime}, g_{\mathbf{K}} \oplus g_{\mathbf{K}}^{\prime}\right) \cong G r\left(T, g_{\mathbf{K}}\right) \star G r\left(T^{\prime}, g_{\mathbf{K}}^{\prime}\right)$ and $\Lambda\left(\left(T \oplus T^{\prime}\right)^{*}\right) \cong$ $\Lambda\left(T^{*}\right) \otimes \Lambda\left(T^{*}\right)$.

This is the analogue at the level of GDCs of well-known multiplicative properties of Clifford algebras.

The above theorems are infinitesimal in nature but one can globalize, or "quantize", them abstractly as follows. Let $M$ be an oriented manifold with $\mathbf{K}$-metric 
$g_{\mathbf{K}}$ and suppose that all structures involved in $g_{\mathbf{K}}$ are parallel for the Levi-Civita connection of the underlying metric. By holonomy arguments (cf. Section 5), the bundles $I\left(T, g_{\mathbf{K}}\right), O\left(T, g_{\mathbf{K}}\right)^{\prime}$ and $G r\left(T, g_{\mathbf{K}}\right)$ are trivialized by their global parallel sections $I\left(M, g_{\mathbf{K}}\right), O\left(M, g_{\mathbf{K}}\right)^{\prime}$ and $\operatorname{Gr}\left(M, g_{\mathbf{K}}\right)$ respectively. The "quantization" map $Q: \Gamma_{M}\left(\operatorname{Hom}\left(T^{*}, T \oplus T^{*}\right)\right) \rightarrow \operatorname{Diff}^{1}(\Lambda)$ is defined as the map that associates to $\sigma \in \Gamma_{M}\left(\operatorname{Hom}\left(T^{*}, T \oplus T^{*}\right)\right)$, the first-order differential operator whose symbol is $\sigma$ (see Section 5 for the details). Restricting to the subspace $I\left(M, g_{\mathbf{K}}\right)$ of parallel sections, one obtains a map $Q: I\left(M, g_{\mathbf{K}}\right) \rightarrow \operatorname{Diff}^{1}(\Lambda)$, which then extends to an algebra homomorphism $Q: T\left(I\left(M, g_{\mathbf{K}}\right)\right) \rightarrow \operatorname{Diff}(\Lambda)$ from the tensor algebra on $I\left(M, g_{\mathbf{K}}\right)$ to the algebra of differential operators in $\Lambda$. If $\lambda_{I\left(M, g_{\mathbf{K}}\right)}^{-1} \in T^{2}\left(I\left(M, g_{\mathbf{K}}\right)\right)$ denotes the dual of the metric $\lambda_{I\left(M, g_{\mathrm{K}}\right)}$, the main theorem of this paper can be stated as follows (cf. Section 5):

Theorem. Let $M$ be an oriented manifold with $\mathbf{K}$-metric $g_{\mathbf{K}}$ such that all structures involved in $g_{\mathbf{K}}$ are parallel for the Levi-Civita connection. Let $\rho: G r\left(M, g_{\mathbf{K}}\right) \rightarrow$ $G L\left(\Gamma_{M}(\Lambda)\right)$ be the action of $\operatorname{Gr}\left(M, g_{\mathbf{K}}\right)$ in the space of real differential forms over $M$.

(i) For $\tilde{g} \in G r\left(M, g_{\mathbf{K}}\right)$ and $g=\pi(\tilde{g}) \in O\left(M, g_{\mathbf{K}}\right)^{\prime}$,

$$
Q(g \circ s)=(-1)^{\operatorname{deg}(\tilde{g})} \rho(\tilde{g}) \circ Q(s) \circ \rho\left(\tilde{g}^{-1}\right) \forall s \in I\left(M, g_{\mathbf{K}}\right) ;
$$

(ii) $Q(x) Q(y)+Q(y) Q(x)=\frac{1}{\operatorname{dim}_{\mathbf{R}} \mathbf{K}} \lambda_{I\left(M, g_{\mathbf{K}}\right)}(x, y) Q\left(\lambda_{I\left(M, g_{\mathbf{K}}\right)}^{-1}\right) \forall x, y \in I\left(M, g_{\mathbf{K}}\right)$.

This theorem implies all classical Hodge identities for Riemannian $(\mathbf{K}=\mathbf{R})$, Kähler $(\mathbf{K}=\mathbf{C})$ and hyper-Kähler $(\mathbf{K}=\mathbf{H})$ manifolds as well as furnishing analogues in arbitrary signature (see Sections 6, 7 and 8). Moreover, this integrated form of the classical identities can be interpreted as defining a multiplicative functor from the category of manifolds with the type of geometric stucture corresponding to $\mathbf{K}$ to the category of $\mathbf{Z}_{2}$-projective representations of an algebraic structure $\left(O_{\mathbf{K}}(1,1), \mathfrak{g}_{\mathbf{K}}\right)$ depending only on $\mathbf{K}$ (cf. 5.9 and 5.11). This functor extends to the category of flat, real graded vector bundles.

Projective representations are not very convenient for practical purposes. However, if $\mathbf{K}=\mathbf{C}$, we give explicit formulae deprojectivising the $\mathbf{Z}_{2}$-projective representation of $\left(O_{\mathbf{C}}(1,1), \mathfrak{g}_{\mathbf{C}}\right)$ in $\Gamma_{M}(\Lambda)$ to a (not always faithful) linear representation of $\left(U(1) \times S L(2, \mathbf{R}), \mathfrak{g}_{\mathbf{C}}\right)$ in $\Gamma_{M}(\Lambda)$. This means, for instance, that each $x \in U(1) \times S L(2, \mathbf{R})$ defines a multiplicative numerical invariant $\left(M, g_{\mathbf{C}}\right) \mapsto$ $\operatorname{Tr}\left(\left.x\right|_{H^{*}(M)}\right)$ of compact Kähler manifolds. From this point of view, the Hodge index formula is a consequence of the fact that conjugate group elements have equal traces (cf. 8.3).

\section{Clifford algebras and Pin Groups}

In this section we first recall the basic properties of Clifford algebras and Pin groups. This is all standard material, and proofs of the various results can be found, for example, in [ABS] or [Sch]. We then associate to every real vector space a Clifford algebra, and prove the basic properties of this association which we will need in the rest of the paper.

1.1 Definition. Let $X$ be a real, finite-dimensional vector space and $B$ a nondegenerate symmetric bilinear form on $X$. The Clifford algebra $C(X, B)$ is the quotient algebra of the tensor algebra $T(X)=\bigoplus X^{\otimes k}$ by the two-sided ideal $\mathfrak{I}$ generated by elements of the form $x \otimes y+y \otimes x-2 B(x, y) 1$, where $x, y \in X$. 
The natural $\mathbf{Z}_{2}$-grading of $T(X)$ factors to a $\mathbf{Z}_{2}$-grading $C(X, B)=C_{+}(X, B) \oplus$ $C_{-}(X, B)$.

One shows that the composition of natural maps $X \rightarrow T(X) \rightarrow C(X, B)$ is an injection; thus we can consider $X$ as embedded in the Clifford algebra, which we will do from now on. If $\left\{e_{1}, e_{2}, \cdots, e_{n}\right\}$ is an orthonormal basis of $X$, the $2^{n}$ elements $\left\{1, e_{i_{1}}, e_{i_{2}}, \cdots, e_{i_{k}}\right\}_{1 \leq i_{1}<\cdots<i_{k} \leq n}$ form a basis of $C(X, B)$ and the rules of calculation are $e_{i} e_{j}+e_{j} e_{i}=2 B\left(e_{i}, e_{j}\right)$.

The Clifford algebra has the following universal property: if $A$ is a real, associative algebra with identity, then any linear map $f: X \rightarrow A$ such that $f(x)^{2}=$ $B(x, x) 1 \forall x \in X$ extends to a unique algebra homomorphism $\tilde{f}: C(X, B) \rightarrow A$. Using the following property and a simple induction argument, one can identify Clifford algebras in terms of matrix algebras:

1.2 Proposition (cf. $\mathrm{ABS})$. If $\left(X_{1}, B_{1}\right)$ and $\left(X_{2}, B_{2}\right)$ are non-degenerate metric spaces, there is a natural isomorphism of $\mathbf{Z}_{2}$-graded algebras

$$
C\left(X_{1}, B_{1}\right) \hat{\otimes} C\left(X_{2}, B_{2}\right) \cong C\left(X_{1} \oplus X_{2}, B_{1} \oplus B_{2}\right) .
$$

The Clifford algebra $C(X, B)$ comes with natural automorphisms and antiautomorphisms with which one can construct a non-trivial double cover of $O(X, B)$.

1.3 Definition. (i) The automorphism of $T(X)$ given by $x_{1} \otimes x_{2} \otimes \cdots \otimes x_{k}$ $\mapsto(-1)^{k} x_{1} \otimes x_{2} \otimes \cdots \otimes x_{k}$ preserves the ideal $\mathfrak{I}$ and so induces an automorphism of $C(X, B)$, which will be denoted $c \mapsto c^{g}$. Then $C(X, B)=C_{+}(X, B) \oplus C_{-}(X, B)=$ $\left\{c \in C(X, B): c=c^{g}\right\} \oplus\left\{c \in C(X, B): c=-c^{g}\right\}$ defines the $\mathbf{Z}_{2}$-grading of the Clifford algebra.

(ii) The antiautomorphism of $T(X)$ given by $x_{1} \otimes x_{2} \otimes \cdots \otimes x_{k} \mapsto x_{k} \otimes \cdots \otimes x_{2} \otimes x_{1}$ preserves the ideal $\mathfrak{I}$ and so induces an antiautomorphism of $C(X, B)$, which will be denoted $c \mapsto c^{T}$.

1.4 Definition/Proposition (cf. $[\mathrm{ABS}]$ ). Let $(X, B)$ be a real, finite-dimensional, non-degenerate metric space and let $C(X, B)$ be its Clifford algebra. The group $\operatorname{Pin}(X, B)$ is defined by

$$
\operatorname{Pin}(X, B)=\left\{c \in C(X, B): c \text { is invertible, } c c^{T}= \pm 1 \quad \text { and } \quad c^{g} X c^{-1}=X\right\},
$$

and $\pi: \operatorname{Pin}(X, B) \rightarrow G L(X)$ is defined by $\pi(c)(x)=c^{g} x c^{-1}$. Then:

(i) $\pi(c)$ is an isometry of $(X, B)$.

(ii) The group Pin $(X, B)$ is a disjoint union

$$
\operatorname{Pin}(X, B)=\operatorname{Pin}_{+}(X, B) \cup \operatorname{Pin}_{-}(X, B)
$$

where

$$
\operatorname{Pin}_{ \pm}(X, B)=\operatorname{Pin}(X, B) \cap C_{ \pm}(X, B) .
$$

We have $\operatorname{det}(\pi(c))= \pm 1$ iff $c \in \operatorname{Pin}_{ \pm}(X, B)$.

(iii) If $x \in X$ satisfies $B(x, x)= \pm 1$, then $x \in$ Pin $_{-}(X, B)$ and $\pi(x) \in O(X, B)$ is the reflection in the hyperplane orthogonal to $x$.

(iv) The map $\pi: \operatorname{Pin}(X, B) \rightarrow O(X, B)$ is a non-trivial $2: 1$ covering map of $O(X, B)$ and $\pi^{-1}(1)=\{ \pm 1\}$.

(v) Every element of $\operatorname{Pin}_{+}(X, B)$ (resp. Pin $\left.(X, B)\right)$ is a product of an even (resp. odd) number of $x$ satisfying (iii). 
1.4.1 Remark. If $g \in O(X, B)$, the action of $g$ on $X$ extends to an automorphism $\alpha_{g}$ of $C(X, B)$ by the universal property of $C(X, B)$. If $\hat{g} \in \operatorname{Pin}(X, B)$ is such that $\pi(\hat{g})=g$, then $\alpha_{g}(c)=(\operatorname{det} g)^{c} \hat{g} c \hat{g}^{-1}$ for all $c \in C(X, B)$.

A finite-dimensional, real vector space $X$ has no preferred non-degenerate symmetric bilinear form. However, duality defines the natural quadratic form $x \oplus \alpha \mapsto$ $\alpha(x)$ on $X \oplus X^{*}$ and hence the natural non-degenerate symmetric bilinear form on $X \oplus X^{*}$ given by

$$
B_{X \oplus X^{*}}(x \oplus \alpha, y \oplus \beta)=\frac{1}{2}(\alpha(y)+\beta(x)) \quad \forall x, y \in X, \forall \alpha, \beta \in X^{*} .
$$

1.5 Definition. Let $X$ be a finite-dimensional real vector space. We denote $C(X \oplus$ $\left.X^{*}, B_{X \oplus X^{*}}\right)$ by $C(X)$.

1.6 Proposition. If $X$ and $X^{\prime}$ are finite-dimensional real vector spaces, there is a natural isomorphism of $\mathbf{Z}_{2}$-graded algebras $F: C(X) \hat{\otimes} C\left(X^{\prime}\right) \rightarrow C\left(X \oplus X^{\prime}\right)$. If $\left\{x_{1}, \cdots, x_{2 n}\right\}$ and $\left\{x_{1}^{\prime}, \cdots, x_{2 m}^{\prime}\right\}$ are bases of $X \oplus X^{*}$ and $X^{\prime} \oplus X^{\prime *}$ (considered as subsets of $\left.X \oplus X^{\prime} \oplus\left(X \oplus X^{\prime}\right)^{*}\right)$ respectively, $F$ is given by concatenation:

$$
F\left(x_{i_{1}} x_{i_{2}} \cdots x_{i_{r}} \otimes x_{j_{1}}^{\prime} x_{j_{2}}^{\prime} \cdots x_{j_{s}}^{\prime}\right)=x_{i_{1}} x_{i_{2}} \cdots x_{i_{r}} x_{j_{1}}^{\prime} x_{j_{2}}^{\prime} \cdots x_{j_{s}}^{\prime} .
$$

Proof. By Proposition 1.2 there is a natural isomorphism of $\mathbf{Z}_{2}$-graded algebras

$$
C(X) \hat{\otimes} C\left(X^{\prime}\right) \cong C\left(\left(X \oplus X^{*}\right) \oplus\left(X^{\prime} \oplus X^{\prime *}\right), B_{X \oplus X^{*}} \oplus B_{X^{\prime} \oplus X^{\prime *}}\right) .
$$

The "permutation" isomorphism,

$$
\left(X \oplus X^{*}\right) \oplus\left(X^{\prime} \oplus X^{\prime *}\right) \cong\left(X \oplus X^{\prime}\right) \oplus\left(X \oplus X^{\prime}\right)^{*},
$$

is clearly an isometry with respect to $B_{X \oplus X^{*}} \oplus B_{X^{\prime} \oplus X^{\prime *}}$ and $B_{\left(X \oplus X^{\prime}\right) \oplus\left(X \oplus X^{\prime}\right)^{*}}$, and hence extends to an isomorphism of the corresponding Clifford algebras. Composing this isomorphism with $(1 \mathrm{~A})$ proves the result.

As for any graded algebra, one has the notion of a graded representation of $C(X)$ :

1.7 Definition. A graded representation (or module) of $C(X)$ in a graded real vector space $\left(E, \varepsilon_{E}\right)$ is a graded algebra homomorphism $\rho: C(X) \rightarrow \operatorname{End}(E)$. (The algebra $\operatorname{End}(E)$ is graded by $f \mapsto \varepsilon_{E} \circ f \circ \varepsilon_{E}$.) By the universal property of Clifford algebras, graded representations in $\left(E, \varepsilon_{E}\right)$ are in bijection with linear maps $f: X \oplus X^{*} \rightarrow \operatorname{End}(E)$ satisfying

$$
\forall x \in X \oplus X^{*}, \quad f(x)^{2}=B(x, x) I d_{E} \quad \text { and } \quad \varepsilon_{E} \circ \rho(x) \circ \varepsilon_{E}=-\rho(x) .
$$

1.7.1 Example. The basic example of a graded $C(X)$ module is the exterior algebra $\Lambda\left(X^{*}\right)$ graded by the degree $\varepsilon_{\Lambda\left(X^{*}\right)}(\omega)=(-1)^{k} \omega$ if $\omega \in \Lambda^{k}\left(X^{*}\right)$. Indeed, if we define $\rho_{\Lambda\left(X^{*}\right)}: X \oplus X^{*} \rightarrow \operatorname{End}\left(\Lambda\left(X^{*}\right)\right)$ by

$$
\begin{aligned}
& \rho_{\Lambda\left(X^{*}\right)}(x)(\omega)=i_{x}(\omega) \quad \text { if } \quad x \in X, \\
& \rho_{\Lambda\left(X^{*}\right)}(\alpha)(\omega)=\alpha \wedge \omega \quad \text { if } \quad \alpha \in X^{*},
\end{aligned}
$$

one has $\rho_{\Lambda\left(X^{*}\right)}(x \oplus \alpha)^{2}=B_{X \oplus X^{*}}(x \oplus \alpha, x \oplus \alpha) I d$, and by the universal property of Clifford algebras, $\rho_{\Lambda\left(X^{*}\right)}$ extends to a homomorphism $\rho_{\Lambda\left(X^{*}\right)}: C(X) \rightarrow$ $\operatorname{End}\left(\Lambda\left(X^{*}\right)\right)$, which is in fact an isomorphism. More generally, if $\left(E, \varepsilon_{E}\right)$ is a graded vector space, then $\left(\Lambda\left(X^{*}\right) \otimes E, \varepsilon_{\Lambda\left(X^{*}\right)} \otimes \varepsilon_{E}\right)$ is a graded $C(X)$ module for the action $c .(\omega \otimes f)=\rho_{\Lambda\left(X^{*}\right)}(c)(\omega) \otimes f$ and every finite-dimensional real graded $C(X)$ module is isomorphic to a graded $C(X)$ module of this form ( $\overline{\mathrm{ABS}}])$. 
Let $\left(E, \varepsilon_{E}, \rho\right)$ and $\left(E^{\prime}, \varepsilon_{E^{\prime}}, \rho^{\prime}\right)$ be respectively graded $C(X)$ and $C\left(X^{\prime}\right)$ modules. The map $\rho \otimes \rho^{\prime}: C(X) \hat{\otimes} C\left(X^{\prime}\right) \rightarrow \operatorname{End}(E) \hat{\otimes} \operatorname{End}\left(E^{\prime}\right)$ given by $\rho \otimes \rho^{\prime}\left(c \otimes c^{\prime}\right)=$ $\rho(c) \otimes \rho^{\prime}\left(c^{\prime}\right)$ is a graded algebra homomorphism. However, one can identify the graded algebras $C(X) \hat{\otimes} C\left(X^{\prime}\right)$ and $C\left(X \oplus X^{\prime}\right)$ by 1.5, and also the graded algebras $\operatorname{End}(E) \hat{\otimes} \operatorname{End}\left(E^{\prime}\right)$ and $\operatorname{End}\left(E \otimes E^{\prime}\right)$ by the map $f \otimes g \mapsto f \hat{\otimes} g$ where

$$
f \hat{\otimes} g\left(e \otimes e^{\prime}\right)=(-1)^{\text {degree }(g) \text { degree }(e)} f(e) \otimes g\left(e^{\prime}\right)
$$

(for homogeneous $f \in \operatorname{End}(E), g \in \operatorname{End}\left(E^{\prime}\right), e \in E$ and $\left.e^{\prime} \in E^{\prime}\right)$. Hence we obtain a graded algebra homomorphism from $C\left(X \oplus X^{\prime}\right)$ to $\operatorname{End}\left(E \otimes E^{\prime}\right)$, i.e., a graded representation of $C\left(X \oplus X^{\prime}\right)$ in $\left(E \otimes E^{\prime}, \varepsilon_{E} \otimes \varepsilon_{E^{\prime}}\right)$.

1.8 Proposition/Definition. Let $(E, \varepsilon, \rho)$ and $\left(E^{\prime}, \varepsilon^{\prime}, \rho^{\prime}\right)$ be respectively graded $C(X)$ and $C\left(X^{\prime}\right)$ modules. There is a unique graded algebra homomorphism $\rho \hat{\otimes} \rho^{\prime}$ : $C\left(X \oplus X^{\prime}\right) \rightarrow \operatorname{End}\left(E \otimes E^{\prime}\right)$ such that the following diagram commutes:

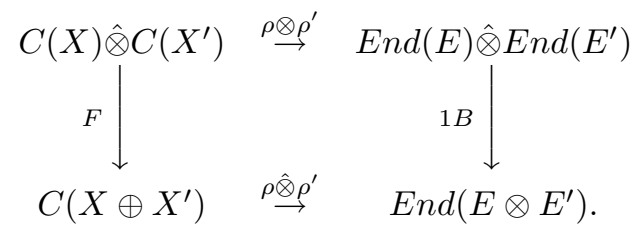

For $x \oplus x^{\prime} \oplus \alpha \oplus \alpha^{\prime} \in\left(X \oplus X^{\prime}\right) \oplus\left(X \oplus X^{\prime}\right)^{*}$ one has:

(1C) $\rho \hat{\otimes} \rho^{\prime}\left(x \oplus x^{\prime} \oplus \alpha \oplus \alpha^{\prime}\right)\left(e \otimes e^{\prime}\right)=\rho(x \oplus \alpha)(e) \otimes e^{\prime}+(-1)^{e} e \otimes \rho^{\prime}\left(x^{\prime} \oplus \alpha^{\prime}\right)\left(e^{\prime}\right)$.

The tensor product $(E, \varepsilon, \rho) \otimes\left(E^{\prime}, \varepsilon^{\prime}, \rho^{\prime}\right)$ is by definition the graded $C\left(X \oplus X^{\prime}\right)$ module $\left(E \otimes E^{\prime}, \varepsilon \otimes \varepsilon^{\prime}, \rho \hat{\otimes} \rho^{\prime}\right)$.

1.8.1 Example. If $X$ and $X^{\prime}$ are finite-dimensional real vector spaces, concatenation defines a canonical isomorphism of graded vector spaces

$$
\Lambda\left(X^{*}\right) \otimes \Lambda\left(X^{\prime *}\right) \cong \Lambda\left(X^{*} \oplus X^{* *}\right)
$$

which, one can check, is also an isomorphism of graded $C\left(X \oplus X^{\prime}\right)$ modules.

To complete this section we define the symbol of a graded representation of $C(X)$, which will be important later on.

1.9 Definition. Let $(E, \varepsilon, \rho)$ be a graded representation of $C(X)$. The symbol $\operatorname{map} \sigma^{E}: \operatorname{Hom}\left(X^{*}, X \oplus X^{*}\right) \rightarrow \operatorname{Hom}\left(X^{*}, \operatorname{End}(E)\right)$ is defined by

$$
\sigma^{E}(f)_{\alpha}=\rho\left(f_{\alpha}\right) \quad \forall \alpha \in X^{*}, \quad \forall f \in \operatorname{Hom}\left(X^{*}, X \oplus X^{*}\right) .
$$

(Here $X \oplus X^{*}$ is regarded as a subset of $C(X)$ and we have written $f_{a}$ for $f(a)$ whenever $f \in \operatorname{Hom}(A, B)$ and $a \in A$.) Note that the definition of the covering map $\pi: \operatorname{Pin}\left(X \oplus X^{*}\right) \rightarrow O\left(X \oplus X^{*}\right)$ (cf. 1.4) implies that, for all $(g, \hat{g}) \in O\left(X \oplus X^{*}\right) \times$ $\operatorname{Pin}\left(X \oplus X^{*}\right)$ such that $\pi(\hat{g})=g$,

$$
\sigma^{E}(g \circ f)_{\alpha}=(-1)^{g} \rho(\hat{g}) \circ \sigma^{E}(f)_{\alpha} \circ \rho\left(\hat{g}^{-1}\right) .
$$

The symbol map is multiplicative for the tensor product defined in 1.8 above.

1.10 Proposition. For $\alpha \in X^{*}, \alpha^{\prime} \in X^{\prime *}, e \in E, e^{\prime} \in E^{\prime}, s \in H o m\left(X^{*}, X \oplus X^{*}\right)$ and $s^{\prime} \in \operatorname{Hom}\left(X^{\prime *}, X^{\prime} \oplus X^{\prime *}\right)$,

$$
\sigma^{E \otimes E^{\prime}}\left(s \oplus s^{\prime}\right)_{\alpha \oplus \alpha^{\prime}}\left(e \otimes e^{\prime}\right)=\sigma^{E}(s)_{\alpha}(e) \otimes e^{\prime}+(-1)^{e} e \otimes \sigma^{E^{\prime}}\left(s^{\prime}\right)_{\alpha^{\prime}}\left(e^{\prime}\right) .
$$

Proof. This is a direct consequence of equations (1C) and (1D). 


\section{Graded Representations of GRAdED DOUble COVERS AND $\mathbf{Z}_{2}$-PROJECTIVE REPRESENTATIONS}

In this section we introduce two (equivalent) categories with multiplication which will enable us to express concisely the main results of this paper.

2.1 Definition. (a) A graded double cover (GDC) of a group $G$ is a pair $(\hat{G}, \chi)$, where $\pi: \hat{G} \rightarrow G$ is a double covering group of $G$ and $\chi: G \rightarrow \mathbf{Z}_{2}$ (the grading) is a group homomorphism. Two GDCs $\left(\hat{G}_{1}, \chi_{1}\right)$ and $\left(\hat{G}_{2}, \chi_{2}\right)$ are equivalent if and only if $\chi_{1}=\chi_{2}$ and $\hat{G}_{1}$ and $\hat{G}_{2}$ are equivalent as double covers. We denote by -1 the non-trivial element of $\pi^{-1}(1)$, which is necessarily in the centre of $\hat{G}$.

(b) Let $(\hat{G}, \chi)$ be a GDC of the group $G$ and let $(V, \varepsilon)$ be a graded vector space. A graded representation of $(\hat{G}, \chi)$ in $(V, \varepsilon)$ is a group homomorphism $\rho: \hat{G} \rightarrow G L(V)$ such that

$$
\begin{aligned}
& \text { (i) } \quad \rho(-1)=-I d_{V} ; \\
& \text { (ii) } \quad \varepsilon \circ \rho(g) \circ \varepsilon^{-1}=\chi(\pi(g)) \rho(g) \quad \forall g \in \hat{G} .
\end{aligned}
$$

(c) The category $\operatorname{Rep}(G r(G))$ of graded representations of GDCs of a group $G$ is defined by

- the objects are pairs $(\tilde{G}, \tilde{V})$ where $\tilde{G}=(\hat{G}, \chi)$ is a GDC of $G$ and $\tilde{V}=(V, \varepsilon, \rho)$ is a graded representation of $\tilde{G}$;

- a morphism in $\operatorname{Mor}\left((\tilde{G}, \tilde{V}),\left(\tilde{G}^{\prime}, \tilde{V}^{\prime}\right)\right)$ is a pair of maps $\psi \in \operatorname{Hom}\left(\hat{G}, \hat{G}^{\prime}\right)$ and $f \in \operatorname{Hom}\left(V, V^{\prime}\right)$ such that

$$
\begin{aligned}
& \text { (i) } \quad f \circ \varepsilon=\varepsilon^{\prime} \circ f \\
& \text { (ii) } \quad \psi \text { is an isomorphism of GDCs; } \\
& \text { (iii) } \quad f \circ \rho(g)=\rho^{\prime}(\psi(g)) \circ f \text { for all } g \in \hat{G} \text {. }
\end{aligned}
$$

2.1.1 Example. The basic non-trivial example of a GDC is $(\operatorname{Pin}(X, B)$, det) (cf. 1.4), which is a GDC of the orthogonal group $O(X, B)$. If $\operatorname{dim} X$ is even, then the complex spin representation with either of its natural gradings is a graded representation of $(\operatorname{Pin}(X, B)$, det).

2.2 Definition (cf. [] $)$. Let $\left(\hat{G}_{1}, \chi_{1}\right)$ and $\left(\hat{G}_{2}, \chi_{2}\right)$ be two GDCs of $G$ and let $\hat{G}_{1} \hat{\times}_{\mathbf{Z}_{2}} \hat{G}_{2}$ be the group whose underlying set is $\hat{G}_{1} \times \hat{G}_{2} /\{(1,1),(-1,-1)\}$ and whose multiplication is

$$
\left[g_{1}, g_{2}\right]\left[h_{1}, h_{2}\right]=\left[g_{1} h_{1},(-1)^{\partial\left(\chi_{1}\left(h_{1}\right)\right) \partial\left(\chi_{2}\left(g_{2}\right)\right)} g_{2} h_{2}\right] .
$$

The product $\left(\hat{G}_{1}, \chi_{1}\right) \star\left(\hat{G}_{2}, \chi_{2}\right)$ is the GDC of $G$ defined by

$$
\left(\hat{G}_{1}, \chi_{1}\right) \star\left(\hat{G}_{2}, \chi_{2}\right)=\left(\left\{\left[g_{1}, g_{2}\right] \in \hat{G}_{1} \hat{\times}_{\mathbf{z}_{2}} \hat{G}_{2}: \pi_{1}\left(g_{1}\right)=\pi_{2}\left(g_{2}\right)\right\}, \chi_{1} \chi_{2}\right) .
$$

In this definition, $\partial: \mathbf{Z}_{2} \rightarrow\{0,1\}$ is the map $\partial(1)=0$ and $\partial(-1)=1$. We will often write $(-1)^{g_{1} g_{2}}$ for $(-1)^{\partial\left(\chi_{1}\left(g_{1}\right)\right) \partial\left(\chi_{2}\left(g_{2}\right)\right)}$ when it is clear which characters $\chi_{1}, \chi_{2}: G \rightarrow \mathbf{Z}_{2}$ are involved. For brevity, we will denote the underlying group of the graded double cover $\left(\hat{G}_{1}, \chi_{1}\right) \star\left(\hat{G}_{2}, \chi_{2}\right)$ also by $\hat{G}_{1} \star \hat{G}_{2}$ even though the definition of the product in this group depends on the choice of characters $\chi_{1}$ and $\chi_{2}$.

The product $\star$ is an analogue for GDCs of the graded tensor product for graded algebras. 
2.3 Proposition. Let $(\hat{G}, \chi),\left(\hat{G}^{\prime}, \chi^{\prime}\right)$ be GDCs of $G$. Let $A, A^{\prime}$ be $\mathbf{Z}_{2}$-graded algebras and let $\rho: \hat{G} \rightarrow A$ and $\rho^{\prime}: \hat{G}^{\prime} \rightarrow A^{\prime}$ be homomorphisms that preserve degree such that $\rho(-1)=-1$ and $\rho^{\prime}(-1)=-1$.

Then $\rho \otimes \rho^{\prime}: \hat{G} \hat{\times} \mathbf{z}_{2} \hat{G}^{\prime} \rightarrow A \hat{\otimes} A^{\prime}$ given by $\rho \otimes \rho^{\prime}\left(\left[g, g^{\prime}\right]\right)=\rho(g) \otimes \rho^{\prime}\left(g^{\prime}\right)$ is a welldefined homomorphism that preserves degree and satisfies $\rho \otimes \rho^{\prime}([-1,1])=-1$.

Proof. Abstract nonsense.

If $(V, \varepsilon, \rho)$ and $\left(V^{\prime}, \varepsilon^{\prime}, \rho^{\prime}\right)$ are graded representations of $(\hat{G}, \chi)$ and $\left(\hat{G}^{\prime}, \chi^{\prime}\right)$ respectively, the maps $\rho$ and $\rho^{\prime}$ satisfy the hypotheses of Proposition 2.3. Hence $\rho \otimes \rho^{\prime}: \hat{G} \hat{\times}_{\mathbf{z}_{2}} \hat{G}^{\prime} \rightarrow \operatorname{End}(V) \hat{\otimes} \operatorname{End}\left(V^{\prime}\right)$ is a homomorphism which, when composed with the graded algebra isomorphism $\operatorname{End}(V) \hat{\otimes} \operatorname{End}\left(V^{\prime}\right) \cong \operatorname{End}\left(V \otimes V^{\prime}\right)$ (cf. equation (1B)), gives a homomorphism $\rho \hat{\otimes} \rho^{\prime}: \hat{G} \hat{\times}_{\mathbf{z}_{2}} \hat{G}^{\prime} \rightarrow \operatorname{End}\left(V \otimes V^{\prime}\right)$ preserving gradings and satisfying $\rho \hat{\otimes} \rho^{\prime}(-1)=-1$.

2.4 Definition. Let $\tilde{G}=(\hat{G}, \chi)$ and $\tilde{G}^{\prime}=\left(\hat{G}^{\prime}, \chi^{\prime}\right)$ be GDC's of $G$ and let $(V, \varepsilon, \rho)$ and $\left(V^{\prime}, \varepsilon^{\prime}, \rho^{\prime}\right)$ be graded representations of $\tilde{G}$ and $\tilde{G}^{\prime}$ respectively. The tensor product $(\tilde{G},(V, \varepsilon, \rho)) \otimes\left(\tilde{G}^{\prime},\left(V^{\prime}, \varepsilon^{\prime}, \rho^{\prime}\right)\right)$ is defined by

$$
(\tilde{G},(V, \varepsilon, \rho)) \otimes\left(\tilde{G}^{\prime},\left(V^{\prime}, \varepsilon^{\prime}, \rho^{\prime}\right)\right)=\left(\tilde{G} \star \tilde{G}^{\prime},\left(V \otimes V^{\prime}, \varepsilon \otimes \varepsilon^{\prime}, \rho \star \rho^{\prime}\right)\right),
$$

where $\rho \star \rho^{\prime}=\left.\left(\rho \hat{\otimes} \rho^{\prime}\right)\right|_{\hat{G} \star \hat{G}^{\prime}}$. Explicitly,

$$
\rho \star \rho^{\prime}\left(\left[g, g^{\prime}\right]\right)\left(v \otimes v^{\prime}\right)=(-1)^{\partial\left(\chi^{\prime}\left(g^{\prime}\right)\right) \partial(v)} \rho(g)(v) \otimes \rho^{\prime}\left(g^{\prime}\right)\left(v^{\prime}\right)
$$

for $g \in \hat{G}$ and $g^{\prime} \in \hat{G}^{\prime}$ satisfying $\pi(g)=\pi^{\prime}\left(g^{\prime}\right)$.

$2.1 \mathbf{Z}_{2}$-projective representations. Let $(V, \varepsilon)$ be a graded vector space. We set

$$
\begin{aligned}
G L_{ \pm}(V) & =\{g \in G L(V): g \circ \varepsilon= \pm \varepsilon \circ g\}, \\
G L(V, \varepsilon) & =G L_{+}(V) \cup G L_{-}(V), \\
P G L(V, \varepsilon) & =G L_{+}(V) \cup G L_{-}(V) /\left\{ \pm I d_{V}\right\}
\end{aligned}
$$

and we denote $\pi_{V}: G L(V, \varepsilon) \rightarrow P G L(V, \varepsilon)$ the projection.

2.5 Definition. A $\mathbf{Z}_{2}$-projective representation of a group $G$ in $(V, \varepsilon)$ is a group homomorphism $r: G \rightarrow P G L(V, \varepsilon)$.

Given a graded representation $((\hat{G}, \chi),(V, \varepsilon, \rho))$ of the GDC $(\hat{G}, \chi)$ of $G$, one can associate to it the $\mathbf{Z}_{2}$-projective representation $[\rho]: G \rightarrow P G L(V, \varepsilon)$ given by

$$
[\rho](g)=\pi_{V}(\rho(\hat{g})) \quad \text { where } g \in G \text { and } \hat{g} \in \hat{G} \text { lifts } g .
$$

Conversely, given a $\mathbf{Z}_{2}$-projective representation $G$ in $(V, \varepsilon)$, one can associate to it the graded representation $\left(\left(r^{*} G L(V, \varepsilon), \varepsilon \circ r\right),(V, \varepsilon, \hat{r})\right)$ of the GDC $\left(r^{*} G L(V, \varepsilon)\right.$, $\varepsilon \circ r)$ of $G$.

With the appropriate definition of morphisms of $\mathbf{Z}_{2}$-projective representations, these maps define an equivalence of categories. This equivalence is an equivalence of categories with multiplication if the product of two $\mathbf{Z}_{2}$-projective representations $(V, \varepsilon, r)$ and $\left(V^{\prime}, \varepsilon^{\prime}, r^{\prime}\right)$ is defined as the vector space $V \otimes V^{\prime}$ with the tensor product grading and the action $r \hat{\otimes} r^{\prime}: G \rightarrow P G L\left(V \otimes V^{\prime}, \varepsilon \otimes \varepsilon^{\prime}\right)$ given by

$$
r \hat{\otimes} r^{\prime}(g)=\left[\widehat{r(g)} \hat{\otimes} \widehat{r^{\prime}(g)}\right],
$$

where $\widehat{r(g)} \in G L(V, \varepsilon)$ (resp. $\left.\widehat{r^{\prime}(g)} \in G L\left(V^{\prime}, \varepsilon^{\prime}\right)\right)$ is any element projecting to $r(g)$ (resp. $\left.r^{\prime}(g)\right)$. 


\section{K-metrics on $T$ And Commuting subgroups of $O\left(T \oplus T^{*}\right)$}

In this section we associate to a real, Hermitian or "quaternion Hermitian" metric space another metric space of the same type, and show that for any two metric spaces of the same type, the associated spaces are canonically isometric. In order to be able to treat the three cases in a uniform way, we introduce the following terminology:

3.1 Definition. Let $T$ be a real vector space and let $\mathbf{K}$ be one of the real algebras $\mathbf{R}, \mathbf{C}$ or $\mathbf{H}$. A (left) $\mathbf{K}$-metric on $T$ is a non-degenerate symmetric bilinear form $g$ on $T$ together with a (left) action $\rho: \mathbf{K} \rightarrow \operatorname{End}(T)$ of $\mathbf{K}$ which is compatible with $g$ in the sense that

$$
g\left(\rho(k) t, t^{\prime}\right)=g\left(t, \rho(\bar{k}) t^{\prime}\right) \quad \forall k \in \mathbf{K}, \forall t, t^{\prime} \in T .
$$

We use the symbols $g_{\mathbf{K}}$ to denote a $\mathbf{K}$-metric (i.e., the couple $\left.(g, \rho)\right)$ and $O\left(T, g_{\mathbf{K}}\right)$ to denote the group of automorphisms of $g_{\mathbf{K}}$ (i.e., the group of isometries of $g$ that commute with $\rho) . O\left(T, g_{\mathbf{K}}\right)$ is an orthogonal, unitary or Sp group depending on whether $\mathbf{K}=\mathbf{R}, \mathbf{C}$ or $\mathbf{H}$ respectively.

3.1.1 Remark. The real dual $T^{*}$ of a $\mathbf{K}$-metric space has a natural $\mathbf{K}$-metric: the bilinear form is $g^{-1}$, the dual metric, and the $\mathbf{K}$-action $\rho^{*}: \mathbf{K} \rightarrow \operatorname{End}\left(T^{*}\right)$ is given by $\rho^{*}(k)(\alpha)(t)=\alpha(\rho(\bar{k})(t))$. One checks that the map $\tau_{g}: T \rightarrow T^{*}$ given by $\tau_{g}(t)\left(t^{\prime}\right)=g\left(t, t^{\prime}\right)$ is an isomorphism of the $\mathbf{K}$-metric spaces $(T, g, \rho)$ and $\left(T^{*}, g^{-1}, \rho^{*}\right)$.

As pointed out in Section 1, $T \oplus T^{*}$ has a natural symmetric non-degenerate bilinear form given by

$$
B\left(t \oplus \alpha, t^{\prime} \oplus \alpha^{\prime}\right)=\frac{1}{2}\left(\alpha\left(t^{\prime}\right)+\alpha^{\prime}(t)\right)
$$

where $\alpha, \alpha^{\prime} \in T^{*}$ and $t, t^{\prime} \in T$. The associated quadratic form is "duality" in the sense that $B(t \oplus \alpha, t \oplus \alpha)=\alpha(t)$. The subspaces $T$ and $T^{*}$ are maximal isotropic and $B$ is of signature $(\operatorname{dim} T, \operatorname{dim} T)$. Denoting by $O\left(T \oplus T^{*}\right)$ the group of isometries of $B$, there is a natural embedding of $G L(T)$ in $O\left(T \oplus T^{*}\right)$ given in block form by

$$
g \mapsto\left(\begin{array}{cc}
g & 0 \\
0 & g^{t^{-1}}
\end{array}\right),
$$

where $g \in G L(T)$ and $g^{t} \in G L\left(T^{*}\right)$ is its transpose.

Now suppose that $T$ has a $\mathbf{K}$-metric $g_{\mathbf{K}}$. The group $O\left(T, g_{\mathbf{K}}\right)$ is a subgroup of $G L(T)$ and hence is naturally embedded in $O\left(T \oplus T^{*}\right)$ as above. The next theorem shows that $O\left(T, g_{\mathbf{K}}\right)^{\prime}$, the commutant of $O\left(T, g_{\mathbf{K}}\right)$ in $O\left(T \oplus T^{*}\right)$, is canonically isomorphic to a fixed matrix group that does not depend on $T$ or $g_{\mathbf{K}}$.

3.2 Definition. Let $O_{\mathbf{K}}(1,1)$ be the group of right $\mathbf{K}$-linear isometries of $\mathbf{K}^{2}$ equipped with the metric $\lambda\left(\left(\begin{array}{l}x \\ y\end{array}\right),\left(\begin{array}{l}x^{\prime} \\ y^{\prime}\end{array}\right)\right)=\operatorname{Re}\left(\frac{1}{2}\left(\bar{x} y^{\prime}+\bar{y} x^{\prime}\right)\right)$. As a group of matrices acting by left multiplication,

$$
O_{\mathbf{K}}(1,1)=\left\{\left(\begin{array}{ll}
a & b \\
c & d
\end{array}\right) \in M_{2}(\mathbf{K}):\left(\begin{array}{cc}
\bar{c} & \bar{a} \\
\bar{d} & \bar{b}
\end{array}\right)\left(\begin{array}{ll}
a & b \\
c & d
\end{array}\right)=\left(\begin{array}{ll}
0 & 1 \\
1 & 0
\end{array}\right)\right\} .
$$

3.3 Theorem. The map $c_{T}: O_{\mathbf{K}}(1,1) \rightarrow O\left(T, g_{\mathbf{K}}\right)^{\prime}$ given by

$$
c_{T}\left(\left(\begin{array}{ll}
a & b \\
c & d
\end{array}\right)\right)=\left(\begin{array}{cc}
\rho(a) & \rho(b) \circ \tau_{g}^{-1} \\
\rho^{*}(c) \circ \tau_{g} & \rho^{*}(d)
\end{array}\right)
$$


is a group isomorphism onto $O\left(T, g_{\mathbf{K}}\right)^{\prime}$. Its restriction to $O_{\mathbf{R}}(1,1)$ is an isomorphism onto $O(T, g)^{\prime}$.

Proof. Writing $x \in O\left(T, g_{\mathbf{K}}\right)^{\prime}$ in block form, $x=\left(\begin{array}{ll}A & B \\ C & D\end{array}\right)$, the fact that $x$ commutes with $O\left(T, g_{\mathbf{K}}\right)$ is equivalent to the fact that $A \in \operatorname{Hom}(T, T), B \in$ $\operatorname{Hom}\left(T^{*}, T\right), C \in \operatorname{Hom}\left(T, T^{*}\right)$ and $D \in \operatorname{Hom}\left(T^{*}, T^{*}\right)$ are $O\left(T, g_{\mathbf{K}}\right)$-equivariant maps. Since the commutant of $O\left(T, g_{\mathbf{K}}\right)$ in $\operatorname{Hom}(T, T)\left(\operatorname{resp} . \operatorname{Hom}\left(T, T^{*}\right)\right)$ is $\rho(\mathbf{K})\left(\right.$ resp. $\left.\rho^{*}(\mathbf{K}) \circ \tau_{g}\right)$, we have

$$
x=\left(\begin{array}{cc}
\rho(a) & \rho(b) \circ \tau_{g}^{-1} \\
\rho^{*}(c) \circ \tau_{g} & \rho^{*}(d)
\end{array}\right),
$$

for some $a, b, c$ and $d$ in $\mathbf{K}$. It is easily seen that $x \in O\left(T \oplus T^{*}\right)$ iff

$$
\left(\begin{array}{cc}
\rho(c)^{*} & \rho(a)^{*} \circ \tau_{g}^{-1} \\
\rho^{*}(d)^{*} \circ \tau_{g} & \rho^{*}(b)^{*}
\end{array}\right)\left(\begin{array}{cc}
\rho(a) & \rho(b) \circ \tau_{g}^{-1} \\
\rho^{*}(c) \circ \tau_{g} & \rho^{*}(d)
\end{array}\right)=\left(\begin{array}{cc}
0 & \tau_{g}^{-1} \\
\tau_{g} & 0
\end{array}\right) .
$$

Using $\rho(a)^{*}=\rho(\bar{a})$ (cf. (3A)), etc. and multiplying out, this reduces to the $2 \times 2$ matrix equation

$$
\left(\begin{array}{ll}
\bar{c} & \bar{a} \\
\bar{d} & \bar{b}
\end{array}\right)\left(\begin{array}{ll}
a & b \\
c & d
\end{array}\right)=\left(\begin{array}{ll}
0 & 1 \\
1 & 0
\end{array}\right) .
$$

This is precisely the condition for $\left(\begin{array}{ll}a & b \\ c & d\end{array}\right)$ to be in $O_{\mathbf{K}}(1,1)$.

There is a natural $\mathbf{K}$-metric space of which $O\left(T, g_{\mathbf{K}}\right)^{\prime}$ is the group of automorphisms. Consider the vector space $I\left(T, g_{\mathbf{K}}\right)=\operatorname{Hom}_{O\left(T, g_{\mathbf{K}}\right)}\left(T^{*}, T \oplus T^{*}\right)$ of $O\left(T, g_{\mathbf{K}}\right)$ equivariant linear maps from $T^{*}$ to $T \oplus T^{*}$. This has the following properties:

(a) The left actions of $\mathbf{K}$ on $T \oplus T^{*}$ and $T^{*}$ commute with $O\left(T, g_{\mathbf{K}}\right)$ and hence induce respectively left and right actions of $\mathbf{K}$ on $I\left(T, g_{\mathbf{K}}\right)$ which commute (if $\mathbf{K}=\mathbf{R}$ or $\mathbf{C}$ they are the same); explicitly, if we represent $f \in \operatorname{Hom}\left(T^{*}, T \oplus T^{*}\right)$ in block form $f=\left(\begin{array}{l}f_{1} \\ f_{2}\end{array}\right)$ with $f_{1} \in \operatorname{Hom}\left(T^{*}, T\right)$ and $f_{2} \in \operatorname{Hom}\left(T^{*}, T^{*}\right)$, then this bimodule structure is

$$
\left(k, f, k^{\prime}\right) \mapsto\left(\begin{array}{c}
\rho(k) \circ f_{1} \circ \rho^{*}\left(k^{\prime}\right) \\
\rho^{*}(k) \circ f_{2} \circ \rho^{*}\left(k^{\prime}\right)
\end{array}\right) .
$$

(b) The group $O\left(T, g_{\mathbf{K}}\right)^{\prime}$ acts naturally by composition on $I\left(T, g_{\mathbf{K}}\right)$ and commutes with the above right action of $\mathbf{K}$ on $I\left(T, g_{\mathbf{K}}\right)$.

(c) For $f, h \in I\left(T, g_{\mathbf{K}}\right)$, the function

$$
\alpha \mapsto B(f(\alpha), h(\alpha))
$$

defines an $O\left(T, g_{\mathbf{K}}\right)$-invariant quadratic form on $T^{*}$ and hence, since $O\left(T, g_{\mathbf{K}}\right)$ acts irreducibly on $T^{*}$, there is a real number $\lambda_{I\left(T, g_{\mathrm{K}}\right)}(f, h)$ such that for all $\alpha \in T^{*}$,

$$
B(f(\alpha), h(\alpha))=\lambda_{I\left(T, g_{\mathbf{K}}\right)}(f, h) g^{-1}(\alpha, \alpha),
$$

where $g^{-1}$ denotes the metric on $T^{*}$ dual to $g$. This defines a symmetric bilinear form $\lambda_{I\left(T, g_{\mathbf{K}}\right)}$ on $I\left(T, g_{\mathbf{K}}\right)$ which is clearly invariant under the action of $O\left(T, g_{\mathbf{K}}\right)^{\prime}$ and compatible with the natural left and right $\mathbf{K}$ actions on $I\left(T, g_{\mathbf{K}}\right)$. In particular, $I\left(T, g_{\mathbf{K}}\right)$ is a right $\mathbf{K}$-metric space. 
3.4 Theorem. (i) There is a unique $O\left(T, g_{\mathbf{K}}\right)^{\prime}$-invariant symmetric bilinear form $\lambda_{I\left(T, g_{\mathbf{K}}\right)}$ on $I\left(T, g_{\mathbf{K}}\right)$ satisfying

$$
B(f(\alpha), h(\alpha))=\lambda_{I\left(T, g_{\mathbf{K}}\right)}(f, h) g^{-1}(\alpha, \alpha) \quad \forall f, h \in I\left(T, g_{\mathbf{K}}\right), \forall \alpha \in T^{*} .
$$

(ii) The left $\mathbf{K}$-linear map $i_{T}: \mathbf{K}^{2} \rightarrow I\left(T, g_{\mathbf{K}}\right)$ given by

$$
i_{T}\left(\left(\begin{array}{l}
1 \\
0
\end{array}\right)\right)=\tau_{g}^{-1} \text { and } i_{T}\left(\left(\begin{array}{l}
0 \\
1
\end{array}\right)\right)=I d_{T^{*}}
$$

is right $\mathbf{K}$-linear and an isometry with respect to the metrics $\lambda$ and $\lambda_{I\left(T, g_{\mathbf{K}}\right)}$. Its restriction to $\mathbf{R}^{2}$ is a real linear isomorphism onto $I(T, g)$.

(iii) The natural action of $O\left(T, g_{\mathbf{K}}\right)^{\prime}$ on $I\left(T, g_{\mathbf{K}}\right)$ defines an isomorphism of $O\left(T, g_{\mathbf{K}}\right)^{\prime}$ with the group of right $\mathbf{K}$-linear isometries of $\lambda_{I\left(T, g_{\mathbf{K}}\right)}$ and is compatible with $c_{T}$ and $i_{T}$ :

$$
i_{T}(g(x))=c_{T}(g)\left(i_{T}(x)\right) \text { if } g \in O_{\mathbf{K}}(1,1) \text { and } x \in \mathbf{K}^{2} .
$$

Proof. Part (i) follows from the discussion above. To prove part (ii) note first that $I\left(T, g_{\mathbf{K}}\right)=H_{O} m_{O\left(T, g_{\mathbf{K}}\right)}\left(T^{*}, T \oplus T^{*}\right) \cong \operatorname{Hom}_{O\left(T, g_{\mathbf{K}}\right)}\left(T^{*}, T\right) \oplus \operatorname{Hom}_{O\left(T, g_{\mathbf{K}}\right)}\left(T^{*}, T^{*}\right) \cong$ $\tau_{g}^{-1} \rho(\mathbf{K}) \oplus \rho^{*}(\mathbf{K})$ and hence the real dimension of $I\left(T, g_{\mathbf{K}}\right)$ is $2 \operatorname{dim}_{\mathbf{R}} \mathbf{K}$. Thus $i_{T}$ is an isomorphism of left $\mathbf{K}$-modules.

To prove that $i_{T}$ is also an isomorphism of right $\mathbf{K}$-modules, one has to show that

$$
i_{T}\left(\left(\begin{array}{l}
k_{1} \\
k_{2}
\end{array}\right) k\right)=i_{T}\left(\left(\begin{array}{l}
k_{1} \\
k_{2}
\end{array}\right)\right) \rho^{*}(k) \quad \forall k_{1}, k_{2}, k \in \mathbf{K} .
$$

In block form, the L.H.S. is $\left(\begin{array}{c}\rho\left(k_{1} k\right) \circ \tau_{g}^{-1} \\ \rho^{*}\left(k_{2} k\right)\end{array}\right)$ and the R.H.S. is $\left(\begin{array}{c}\rho\left(k_{1}\right) \circ \tau_{g}^{-1} \circ \rho^{*}(k) \\ \rho^{*}\left(k_{2}\right) \circ \rho^{*}(k)\end{array}\right)$ (cf. 3B). These two quantities are equal since $\tau_{g}^{-1}: T^{*} \rightarrow T$ is an isomorphism of $\mathbf{K}$-modules. Part (iii) follows by direct calculation with the explicit formulae for $c_{T}$ and $i_{T}$.

3.5 Proposition. If $\tilde{d}: O\left(T \oplus T^{*}\right) \times O\left(T^{\prime} \oplus T^{\prime *}\right) \rightarrow O\left(\left(T \oplus T^{\prime}\right) \oplus\left(T \oplus T^{\prime}\right)^{*}\right)$ and $\tilde{d}_{1}: \operatorname{Hom}\left(T^{*}, T \oplus T^{*}\right) \times \operatorname{Hom}\left(T^{\prime *}, T^{\prime} \oplus T^{\prime *}\right) \rightarrow \operatorname{Hom}\left(\left(T \oplus T^{\prime}\right)^{*},\left(T \oplus T^{\prime}\right) \oplus\left(T \oplus T^{\prime}\right)^{*}\right)$ are the natural embeddings, then for $g \in O_{\mathbf{K}}(1,1)$ and $x \in \mathbf{K}^{2}$,

$$
\tilde{d}\left(c_{T}(g), c_{T^{\prime}}(g)\right)=c_{T \oplus T^{\prime}}(g) \quad \text { and } \quad \tilde{d}_{1}\left(i_{T}(x), i_{T^{\prime}}(x)\right)=i_{T \oplus T^{\prime}}(x) .
$$

Proof. Straightforward.

\section{Multiplicativity OF GRADED DOUble COVERS}

In this section we associate to a $\mathbf{K}$-metric space $\left(T, g_{\mathbf{K}}\right)$ a GDC of the matrix group $O_{\mathbf{K}}(1,1)$, and show that this association is multiplicative in an appropriate sense.

Recall that the group $\operatorname{Pin}\left(T \oplus T^{*}, B\right)$, is a non-trivial double cover of $O\left(T \oplus T^{*}\right)$. The pair $\left(\operatorname{Pin}\left(T \oplus T^{*}, B\right)\right.$, det $)$, where det $: O\left(T \oplus T^{*}\right) \rightarrow \mathbf{Z}_{2}$ is the determinant homomorphism, is a graded double cover (GDC) of $O\left(T \oplus T^{*}\right)$ (cf. Definition 2.1), which we denote by ${ }^{\varepsilon} \operatorname{Pin}\left(T \oplus T^{*}, B\right)$.

4.1 Definition. Let $\left(T, g_{\mathbf{K}}\right)$ be a finite-dimensional $\mathbf{K}$-metric space. Define $\operatorname{Gr}\left(T, g_{\mathbf{K}}\right)$ to be the GDC of $O\left(T, g_{\mathbf{K}}\right)^{\prime}$ given by

$$
\operatorname{Gr}\left(T, g_{\mathbf{K}}\right)=\left(\pi^{-1}\left(O\left(T, g_{\mathbf{K}}\right)^{\prime}\right),\left.\operatorname{det}\right|_{O\left(T, g_{\mathbf{K}}\right)^{\prime}}\right) .
$$


This can also be considered as a GDC of the matrix group $O_{\mathbf{K}}(1,1)$ with the projection $c_{T}^{-1} \circ \pi: \pi^{-1}\left(O\left(T, g_{\mathbf{K}}\right)^{\prime}\right) \rightarrow O_{\mathbf{K}}(1,1)$ (cf. 3.3).

4.1.1 Remark. If $\mathbf{K}=\mathbf{C}$ or $\mathbf{K}=\mathbf{H}$, the group $O\left(T, g_{\mathbf{K}}\right)^{\prime}$, respectively isomorphic to $U(1,1)$ or $S p(1,1)$, is a connected subgroup of $O\left(T \oplus T^{*}\right)$ and hence $\left.\operatorname{det}\right|_{O\left(T, g_{\mathbf{K}}\right)^{\prime}}=1$ is the trivial grading.

4.1.2 Remark. A priori, the groups $\pi^{-1}\left(O\left(T, g_{\mathbf{K}}\right)^{\prime}\right)$ and $\pi^{-1}\left(O\left(T, g_{\mathbf{K}}\right)\right)$ commute only up to sign. If $\mathbf{K}=\mathbf{R}$, then they do not commute but $\pi^{-1}\left(O\left(T, g_{\mathbf{K}}\right)^{\prime}\right)$ and $\pi^{-1}\left(S O\left(T, g_{\mathbf{K}}\right)\right)$ commute (cf. 3.4(ii) in $\underline{\text { S }}$ ). If $\mathbf{K}=\mathbf{C}$ or $\mathbf{K}=\mathbf{H}$, then $O\left(T, g_{\mathbf{K}}\right)^{\prime}$ is connected and hence, since any point is joined by a continuous path to either 1 or -1 , then $\pi^{-1}\left(O\left(T, g_{\mathbf{K}}\right)^{\prime}\right)$ and $\pi^{-1}\left(O\left(T, g_{\mathbf{K}}\right)\right)$ commute. Hence, in all cases,

$$
\operatorname{Gr}\left(T, g_{\mathbf{K}}\right) \subset C(T)^{S O\left(T, g_{\mathbf{K}}\right)},
$$

where $C(T)^{S O\left(T, g_{\mathbf{K}}\right)}$ denotes the fixed point set of $S O\left(T, g_{\mathbf{K}}\right)$ acting on $C(T)$ by $\alpha$ (cf. 1.4.1).

4.1.3 Remark. By Theorem 3.3, the group $O\left(T, g_{\mathbf{K}}\right)^{\prime}$ is independent of the $\mathbf{K}$-metric space $\left(T, g_{\mathbf{K}}\right)$ but, as we shall see later, the same is not true for $\operatorname{Gr}\left(T, g_{\mathbf{K}}\right)$ unless $\mathbf{K}=\mathbf{H}$. However, the map $\pi_{*}{ }^{-1} \circ c_{T *}: o_{\mathbf{K}}(1,1) \rightarrow \operatorname{gr}\left(T, g_{\mathbf{K}}\right)$ is an isomorphism of Lie algebras. Hence the Lie algebra of $\operatorname{Gr}\left(T, g_{\mathbf{K}}\right)$ is canonically isomorphic to $\mathbf{R}$ if $\mathbf{K}=\mathbf{R}$, to $u(1,1) \cong \mathbf{R} \oplus \operatorname{sl}(2, \mathbf{R})$ if $\mathbf{K}=\mathbf{C}$ and to $s p(1,1)$ if $\mathbf{K}=\mathbf{H}$.

The main result of this section will be that $\operatorname{Gr}\left(T, g_{\mathbf{K}}\right)$ as a $\operatorname{GDC}$ of $O_{\mathbf{K}}(1,1)$ is multiplicative with respect to direct sums of $\mathbf{K}$-metric spaces. To see this, let $\left(T, g_{\mathbf{K}}\right)$ and $\left(T^{\prime}, g_{\mathbf{K}}^{\prime}\right)$ be $\mathbf{K}$-metric spaces. By definition, the inclusions $j_{T}: \operatorname{Pin}(T \oplus$ $\left.T^{*}, B\right) \hookrightarrow C(T)$ and $j_{T^{\prime}}: \operatorname{Pin}\left(T^{\prime} \oplus T^{* *}, B^{\prime}\right) \hookrightarrow C\left(T^{\prime}\right)$ are compatible with gradings and satisfy $j_{T}(-1)=-1$ and $j_{T^{\prime}}(-1)=-1$. Hence by Proposition 2.3 we can define an injective graded homomorphism

$$
j_{T} \otimes j_{T^{\prime}}: \quad{ }^{\varepsilon} \operatorname{Pin}\left(T \oplus T^{*}, B\right) \hat{\times} \mathbf{z}_{2}{ }^{\varepsilon} \operatorname{Pin}\left(T^{\prime} \oplus T^{\prime *}, B^{\prime}\right) \hookrightarrow C(T) \hat{\otimes} C\left(T^{\prime}\right) .
$$

Composing with the isomorphism of Proposition 1.6 we get an injective graded homomorphism:

$$
F \circ j_{T} \otimes j_{T^{\prime}}: \quad{ }^{\varepsilon} \operatorname{Pin}\left(T \oplus T^{*}, B\right) \hat{\times} \mathbf{z}_{2}{ }^{\varepsilon} \operatorname{Pin}\left(T^{\prime} \oplus T^{\prime *}, B^{\prime}\right) \hookrightarrow C\left(T \oplus T^{\prime}\right) .
$$

Recall that, by definition, the underlying group of $\operatorname{Gr}\left(T, g_{\mathbf{K}}\right) \star G r\left(T^{\prime}, g_{\mathbf{K}}^{\prime}\right)$ is a graded subgroup of $\operatorname{Pin}\left(T \oplus T^{*}, B\right) \times \mathbf{z}_{2} \operatorname{Pin}\left(T^{\prime} \oplus T^{* *}, B^{\prime}\right)$ and the underlying group of $G r\left(T \oplus T^{\prime}, g_{\mathbf{K}} \oplus g_{\mathbf{K}}^{\prime}\right)$ is a graded subgroup of $C\left(T \oplus T^{\prime}\right)$.

4.2 Theorem. There is a unique isomorphism $f: G r\left(T, g_{\mathbf{K}}\right) \star G r\left(T^{\prime}, g_{\mathbf{K}}^{\prime}\right) \rightarrow$ $G r\left(T \oplus T^{\prime}, g_{\mathbf{K}} \oplus g_{\mathbf{K}}^{\prime}\right)$ of $G D C$ s of $O_{\mathbf{K}}(1,1)$ such that the following diagram commutes:

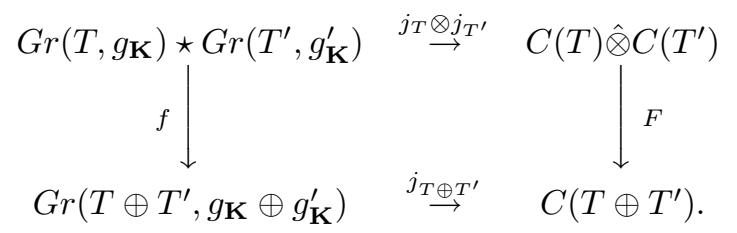

Proof. This result is very closely related to the results of Section 2 in [S]. In order to be able to apply them, we first compose $j_{T} \otimes j_{T^{\prime}}: \operatorname{Pin}\left(T \oplus T^{*}, B\right) \hat{\times} \mathbf{z}_{2} \operatorname{Pin}\left(T^{\prime} \oplus\right.$ 
$\left.T^{*}, B^{\prime}\right) \hookrightarrow C(T) \hat{\otimes} C\left(T^{\prime}\right)$ with the isomorphism $C(T) \hat{\otimes} C\left(T^{\prime}\right) \cong C\left(\left(T \oplus T^{*}\right) \oplus\left(T^{\prime} \oplus\right.\right.$ $\left.\left.T^{\prime *}\right), B \oplus B^{\prime}\right)$ given by equation (1A) to obtain an injective homomorphism:

$$
\begin{aligned}
D & =(1 A) \circ j_{T} \otimes j_{T^{\prime}}: \operatorname{Pin}\left(T \oplus T^{*}, B\right) \hat{\times} \mathbf{z}_{2} \operatorname{Pin}\left(T^{\prime} \oplus T^{\prime *}, B^{\prime}\right) \\
& \hookrightarrow C\left(\left(T \oplus T^{*}\right) \oplus\left(T^{\prime} \oplus T^{\prime *}\right), B \oplus B^{\prime}\right) .
\end{aligned}
$$

By Lemma 2.5 in $\left[\underline{S}\right.$, the image of $D$ is contained in $\operatorname{Pin}\left(\left(T \oplus T^{*}\right) \oplus\left(T^{\prime} \oplus T^{\prime *}\right), B \oplus B^{\prime}\right)$ and there is a commutative diagram of graded group homomorphisms:

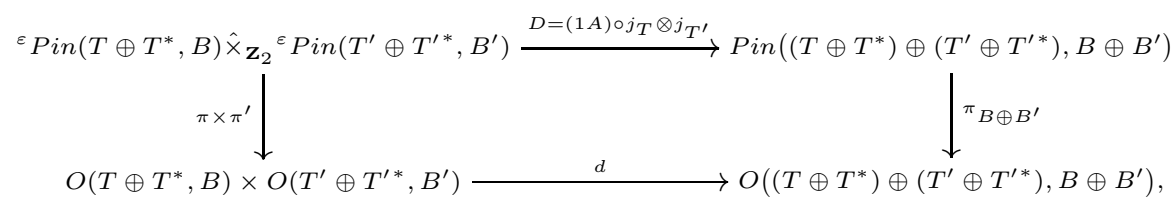

where $d: O\left(T \oplus T^{*}, B\right) \times O\left(T^{\prime} \oplus T^{\prime *}, B^{\prime}\right) \rightarrow O\left(\left(T \oplus T^{*}\right) \oplus\left(T^{\prime} \oplus T^{\prime *}\right), B \oplus B^{\prime}\right)$ is the diagonal injection $d(a, b)=\left(\begin{array}{ll}a & 0 \\ 0 & b\end{array}\right) \in O\left(\left(T \oplus T^{*}\right) \oplus\left(T^{\prime} \oplus T^{\prime *}\right), B \oplus B^{\prime}\right)$. Using the "permutation" isometry

$$
\left.\left(\left(T \oplus T^{*}\right) \oplus\left(T^{\prime} \oplus T^{*}\right), B \oplus B^{\prime}\right) \cong\left(\left(T \oplus T^{\prime}\right) \oplus\left(T \oplus T^{\prime}\right)^{*}\right), B_{T \oplus T^{\prime}}\right),
$$

one can identify the GDC ${ }^{\varepsilon} \operatorname{Pin}\left(\left(T \oplus T^{*}\right) \oplus\left(T^{\prime} \oplus T^{*}\right), B \oplus B^{\prime}\right)$ of $O\left(\left(T \oplus T^{*}\right) \oplus\right.$ $\left.\left(T^{\prime} \oplus T^{*}\right), B \oplus B^{\prime}\right)$ with the GDC $\left.{ }^{\varepsilon} \operatorname{Pin}\left(\left(T \oplus T^{\prime}\right) \oplus\left(T \oplus T^{\prime}\right)^{*}\right), B_{T \oplus T^{\prime}}\right)$ of $O((T \oplus$ $\left.\left.\left.T^{\prime}\right) \oplus\left(T \oplus T^{\prime}\right)^{*}\right), B_{T \oplus T^{\prime}}\right)$ and this gives the commutative diagram

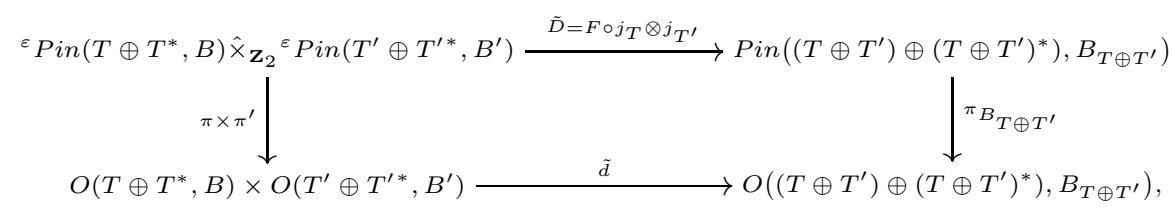

where $\tilde{d}$ is the diagonal embedding $d$ composed with the permutation isometry. Now let $c_{T}: O_{\mathbf{K}}(1,1) \rightarrow O\left(T, g_{\mathbf{K}}\right)^{\prime}$ denote the canonical identification of Theorem 3.3. By Proposition 3.5,

$$
\tilde{d}\left(c_{T}(x), c_{T^{\prime}}(x)\right)=c_{T \oplus T^{\prime}}(x) \quad \forall x \in O_{\mathbf{K}}(1,1) .
$$

The underlying group of the GDC $\operatorname{Gr}\left(T, g_{\mathbf{K}}\right) \star G r\left(T^{\prime}, g_{\mathbf{K}}^{\prime}\right)$ is by definition

$$
\left\{\left[g, g^{\prime}\right] \in{ }^{\varepsilon} \operatorname{Pin}\left(T \oplus T^{*}, B\right) \hat{\mathbf{x}}_{\mathbf{z}_{2}}{ }^{\varepsilon} \operatorname{Pin}\left(T^{\prime} \oplus T^{\prime *}, B^{\prime}\right): c_{T}^{-1} \circ \pi(g)=c_{T^{\prime}}^{-1} \circ \pi^{\prime}\left(g^{\prime}\right)\right\}
$$

with double covering projection $c_{T}^{-1} \circ \pi=c_{T^{\prime}}^{-1} \circ \pi^{\prime}$. On the other hand, the underlying group of the GDC $G r\left(T \oplus T^{\prime}, g_{\mathbf{K}} \oplus g_{\mathbf{K}}^{\prime}\right)$ is by definition $\pi_{B_{T \oplus T^{\prime}}}^{-1}\left(O\left(T \oplus T^{\prime}, g_{\mathbf{K}} \oplus g_{\mathbf{K}}^{\prime}\right)^{\prime}\right)$ with double covering projection $c_{T \oplus T^{\prime}}^{-1} \circ \pi_{B_{T \oplus T^{\prime}}}$. We now show that the restriction of $\tilde{D}$ to the underlying group of $\operatorname{Gr}\left(T, g_{\mathbf{K}}\right) \star G r\left(T^{\prime}, g_{\mathbf{K}}^{\prime}\right)$ is a group isomorphism onto the underlying group of $\operatorname{Gr}\left(T \oplus T^{\prime}, g_{\mathbf{K}} \oplus g_{\mathbf{K}}^{\prime}\right)$ which commutes with covering projections, and therefore, since $\tilde{D}$ preserves gradings, defines an isomorphism of GDCs of $O_{\mathbf{K}}(1,1)$. 
If $\left[g, g^{\prime}\right] \in G r\left(T, g_{\mathbf{K}}\right) \star G r\left(T^{\prime}, g_{\mathbf{K}}^{\prime}\right)$, we have

$$
\begin{aligned}
& \pi_{B_{T \oplus T^{\prime}} \circ \tilde{D}\left(\left[g, g^{\prime}\right]\right)=} \tilde{d} \circ\left(\pi \times \pi^{\prime}\right)\left(\left[g, g^{\prime}\right]\right) \\
&=\tilde{d}\left(\pi(g), \pi^{\prime}\left(g^{\prime}\right)\right) \\
&=\tilde{d}\left(c_{T}(x), c_{T^{\prime}}(x)\right) \\
&\left(\text { where } \quad x=c_{T}^{-1}(\pi(g))=c_{T^{\prime}}^{-1}\left(\pi^{\prime}\left(g^{\prime}\right)\right) \in O_{\mathbf{K}}(1,1)\right) \\
&= c_{T \oplus T^{\prime}}(x) \quad(\text { by }(4 A)) .
\end{aligned}
$$

Thus $\pi_{B_{T \oplus T^{\prime}}} \circ \tilde{D}\left(\left[g, g^{\prime}\right]\right) \in O\left(T \oplus T^{\prime}, g_{\mathbf{K}} \oplus g_{\mathbf{K}}^{\prime}\right)^{\prime}$ and $\tilde{D}$ maps $\operatorname{Gr}\left(T, g_{\mathbf{K}}\right) \star G r\left(T^{\prime}, g_{\mathbf{K}}^{\prime}\right)$ into $\operatorname{Gr}\left(T \oplus T^{\prime}, g_{\mathbf{K}} \oplus g_{\mathbf{K}}^{\prime}\right)$. Furthermore, $c_{T \oplus T^{\prime}}^{-1} \circ \pi_{B_{T \oplus T^{\prime}}} \circ \tilde{D}=c_{T}^{-1} \circ \pi$ and hence $\tilde{D}: \operatorname{Gr}\left(T, g_{\mathbf{K}}\right) \star G r\left(T^{\prime}, g_{\mathbf{K}}^{\prime}\right) \rightarrow G r\left(T \oplus T^{\prime}, g_{\mathbf{K}} \oplus g_{\mathbf{K}}^{\prime}\right)$ commutes with the covering projections.

If $\left(T, g_{\mathbf{K}}\right)$ is a $\mathbf{K}$-metric space, $\operatorname{Gr}\left(T, g_{\mathbf{K}}\right)$ is a graded subgroup of $C(T)$, and hence a graded $C(T)$ module restricts to a graded representation of $G r\left(T, g_{\mathbf{K}}\right)$ in the sense of Definition 2.1(c). The tensor product of restrictions is isomorphic to the restriction of the tensor product:

4.5 Corollary. Let $(E, \varepsilon, \rho)$ and $\left(E^{\prime}, \varepsilon^{\prime}, \rho^{\prime}\right)$ be graded representations of $C(T)$ and $C\left(T^{\prime}\right)$ respectively. Let $\left(E \otimes E^{\prime}, \varepsilon \otimes \varepsilon^{\prime}, \rho \hat{\otimes} \rho^{\prime}\right)$ be the tensor product $C\left(T \oplus T^{\prime}\right)$ representation. The pair $\left(f, I d_{E \otimes E^{\prime}}\right)$ is an isomorphism

$$
\begin{aligned}
& \left(G r\left(T, g_{\mathbf{K}}\right),\left(E, \varepsilon,\left.\rho\right|_{G r\left(T, g_{\mathbf{K}}\right)}\right)\right) \otimes\left(G r\left(T^{\prime}, g_{\mathbf{K}}^{\prime}\right),\left(E^{\prime}, \varepsilon^{\prime},\left.\rho^{\prime}\right|_{G r\left(T^{\prime}, g_{\mathbf{K}}^{\prime}\right)}\right)\right) \\
& \quad \cong\left(G r\left(T \oplus T^{\prime}, g_{\mathbf{K}} \oplus g_{\mathbf{K}}^{\prime}\right),\left(E \otimes E^{\prime}, \varepsilon \otimes \varepsilon^{\prime},\left.\rho \hat{\otimes} \rho^{\prime}\right|_{G r\left(T \oplus T^{\prime}, g_{\mathbf{K}} \oplus g_{\mathbf{K}}^{\prime}\right)}\right)\right)
\end{aligned}
$$

of graded representations of $G D C s$ of $O_{\mathbf{K}}(1,1)$.

Proof. This follows directly from Definitions 1.8(b), 2.4 and the commutative diagram

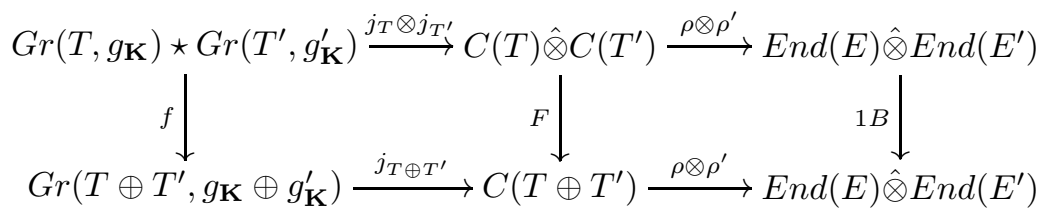

(cf. 4.3 and 1.8(a)).

\section{Manifolds with K-Metrics}

In this section we globalize the main results of Sections 3 and 4 , introduce the quantization map and derive a universal, multiplicative form of Hodge identities.

Let $M$ be a connected $n$-manifold with tangent bundle $T \rightarrow M$ and cotangent bundle $T^{*} \rightarrow M$. Let $g_{\mathbf{K}}=(g, \rho)$ be a $\mathbf{K}$-metric, i.e., $g$ is a non-degenerate metric (of arbitrary signature $(r, s)), \rho: \mathbf{K} \rightarrow \Gamma(\operatorname{End}(T))$ is a real algebra homomorphism and $g$ and $\rho$ are pointwise compatible in the sense of Definition 3.1.

At each point $m \in M$ we can form the group $O\left(T_{m} \oplus T_{m}^{*}\right)$, its subgroups $O\left(T_{m}, g_{\mathbf{K}_{m}}\right)$ and $O\left(T_{m}, g_{\mathbf{K}_{m}}\right)^{\prime}$, and the algebra $C\left(T_{m}\right)$ and its subgroups $\operatorname{Pin}\left(T_{m} \oplus\right.$ $T_{m}^{*}$ ) and $\operatorname{Gr}\left(T_{m}, g_{\mathbf{K}_{m}}\right)$. As $m$ varies in $M$, these spaces fit together to form fibre bundles over $M$, which we denote in the obvious way $O\left(T \oplus T^{*}\right) \rightarrow M$, etc. The bundles $O\left(T \oplus T^{*}\right)$ and $C(T)$ are bundles associated to the principal $G L(n)$ bundle of linear frames in $T$, whereas the bundles $O\left(T, g_{\mathbf{K}}\right) \subseteq O\left(T \oplus T^{*}\right)$, 
$O\left(T, g_{\mathbf{K}}\right)^{\prime} \subseteq O\left(T \oplus T^{*}\right)$ and $G r\left(T, g_{\mathbf{K}}\right) \subseteq C(T)$, whose definition depends on the metric, are associated to $O_{\mathbf{K}}(M)$, the principal bundle of $\mathbf{K}$-orthonormal frames over $M$.

Now suppose that $\nabla$ is a linear connection in $T$ which preserves the $\mathbf{K}$-metric $g_{\mathbf{K}}$, i.e., $\nabla$ preserves $g$ and $\rho$. We will also denote by $\nabla$ the canonically associated linear connections in $T \oplus T^{*}, \operatorname{End}\left(T \oplus T^{*}\right)$ and $C(T)$. Since $\nabla$ preserves $g_{\mathbf{K}}$, it is clear that parallel transport in $E n d\left(T \oplus T^{*}\right)$ preserves the sub-bundles $O\left(T, g_{\mathbf{K}}\right)$ and $O\left(T, g_{\mathbf{K}}\right)^{\prime}$, and that parallel transport in $C(T)$ preserves the sub-bundle $\operatorname{Gr}\left(T, g_{\mathbf{K}}\right)$.

5.1 Proposition. Let $O\left(M, g_{\mathbf{K}}\right)^{\prime}, I\left(M, g_{\mathbf{K}}\right)$ and $G r\left(M, g_{\mathbf{K}}\right)$ denote the parallel sections of $O\left(T, g_{\mathbf{K}}\right)^{\prime}, H_{\mathrm{Hom}}\left(T, g_{\mathbf{K}}\right)\left(T^{*}, T \oplus T^{*}\right)$ and $G r\left(T, g_{\mathbf{K}}\right)$ respectively.

(i) The map $c_{M}: O_{\mathbf{K}}(1,1) \rightarrow O\left(M, g_{\mathbf{K}}\right)^{\prime}$ defined by

$$
c_{M}\left(\left(\begin{array}{ll}
a & b \\
c & d
\end{array}\right)\right)=\left(\begin{array}{cc}
\rho(a) & \rho(b) \circ \tau_{g}^{-1} \\
\rho^{*}(c) \circ \tau_{g} & \rho^{*}(d)
\end{array}\right)
$$

is a group isomorphism. For all $m \in M$, the evaluation map $O\left(M, g_{\mathbf{K}}\right)^{\prime} \rightarrow$ $O\left(T_{m}, g_{\mathbf{K}_{m}}\right)^{\prime}$ is a group isomorphism.

(ii) The left $\mathbf{K}$-linear map $i_{M}: \mathbf{K}^{2} \rightarrow I\left(M, g_{\mathbf{K}}\right)$ given by

$$
i_{M}\left(\left(\begin{array}{l}
1 \\
0
\end{array}\right)\right)=\tau_{g}^{-1} \text { and } i_{M}\left(\left(\begin{array}{l}
0 \\
1
\end{array}\right)\right)=I d_{T^{*}}
$$

is an isomorphism of $\mathbf{K}$-bimodules. If $\lambda_{I\left(M, g_{\mathbf{K}}\right)}$ denotes the metric $i_{M{ }_{*}} \lambda$ on $I\left(M, g_{\mathbf{K}}\right)$, then for all $m \in M$, the evaluation map $I\left(M, g_{\mathbf{K}}\right) \rightarrow I\left(T_{m}, g_{\mathbf{K}_{m}}\right)$ is an isometry with respect to $\lambda_{I\left(M, g_{\mathbf{K}}\right)}$ and $\lambda_{I\left(T_{m}, g_{\mathbf{K}_{m}}\right)}$, and an isomorphism of $\mathbf{K}$ bimodules.

(iii) If $\mathbf{K}=\mathbf{R}$ and $M$ is orientable, then for all $m \in M$ the evaluation map $\operatorname{Gr}\left(M, g_{\mathbf{K}}\right) \rightarrow \operatorname{Gr}\left(T_{m}, g_{\mathbf{K}_{m}}\right)$ is a group isomorphism.

(iv) If $\mathbf{K}=\mathbf{C}$ or $\mathbf{K}=\mathbf{H}$, then for all $m \in M$ the evaluation map $\operatorname{Gr}\left(M, g_{\mathbf{K}}\right) \rightarrow$ $\operatorname{Gr}\left(T_{m}, g_{\mathbf{K}_{m}}\right)$ is a group isomorphism.

(v) There is a unique group homomorphism $d_{M}: O\left(M, g_{\mathbf{K}}\right)^{\prime} \rightarrow \mathbf{Z}_{2}$ such that $d_{M}(s)=\operatorname{det} s(m) \forall m \in M, \forall s \in O\left(M, g_{\mathbf{K}}\right)^{\prime}$, where det $: O\left(T \oplus T^{*}\right) \rightarrow \mathbf{Z}_{2}$ is the determinant.

Proof. Since for all $k \in \mathbf{K}, \rho(k), \rho^{*}(k), \tau_{g}$ and $\tau_{g}^{-1}$ are parallel, the image of $c_{M}$ is contained in $O\left(M, g_{\mathbf{K}}\right)^{\prime}$ and by Theorem 3.3, for all $m \in M, x \in O_{\mathbf{K}}(1,1)$, $c_{M}(x)(m)=c_{T_{m}}(x)$. The map $c_{T_{m}}: O_{\mathbf{K}}(1,1) \rightarrow O\left(T_{m}, g_{\mathbf{K}_{m}}\right)^{\prime}$ is a group isomorphism by Theorem 3.3, which means that for any $y \in O\left(T_{m}, g_{\mathbf{K}_{m}}\right)^{\prime}$ there is a parallel section $c_{M} \circ c_{T_{m}}^{-1}(y)$ of $O\left(T, g_{\mathbf{K}}\right)^{\prime}$ taking the value $y$ at $m$. This proves part (i). The proof of part (ii), which we omit, is analogous.

By 4.1.2 we have

$$
G r\left(T, g_{\mathbf{K}}\right) \subseteq(C(T))^{S O\left(T, g_{\mathbf{K}}\right)} .
$$

If $\mathbf{K}=\mathbf{R}$ and $M$ is orientable, or if $\mathbf{K}=\mathbf{C}$ or $\mathbf{H}, \nabla$ reduces to an $S O(r, s)$ connection and standard properties of holonomy imply that $(C(T))^{S O\left(T, g_{\mathrm{K}}\right)}$ is trivialized by its global parallel sections. The sub-bundle $\operatorname{Gr}\left(T, g_{\mathbf{K}}\right)$ is stable under parallel transport and hence also trivialized by its global parallel sections. This proves parts (iii) and (iv).

Finally, to prove part (v) note that since det $: O\left(T \oplus T^{*}\right) \rightarrow \mathbf{Z}_{2}$ and $s \in O\left(M, g_{\mathbf{K}}\right)^{\prime}$ are parallel, we have $\operatorname{det} s(m)=\operatorname{det} s\left(m^{\prime}\right) \forall m, m^{\prime} \in M$. 
The upshot of this theorem is that one can associate to any (orientable) manifold with $\mathbf{K}$-metric $\left(M, g_{\mathbf{K}}\right)$ a group $O\left(M, g_{\mathbf{K}}\right)^{\prime}$, a $\mathbf{K}$-bimodule $I\left(M, g_{\mathbf{K}}\right)$ with right $\mathbf{K}$ metric $\lambda_{I\left(M, g_{\mathbf{K}}\right)}$ and a GDC $G r\left(M, g_{\mathbf{K}}\right)$ of $O\left(M, g_{\mathbf{K}}\right)^{\prime}$. Furthermore, $O\left(M, g_{\mathbf{K}}\right)^{\prime}$ and $\left(I\left(M, g_{\mathbf{K}}\right), \lambda_{I\left(M, g_{\mathbf{K}}\right)}\right)$ are canonically isomorphic to the model spaces $O_{\mathbf{K}}(1,1)$ and $\left(\mathbf{K}^{2}, \lambda\right)$ so that $\operatorname{Gr}\left(M, g_{\mathbf{K}}\right)$ can be considered as a GDC of the matrix group $O_{\mathbf{K}}(1,1)$.

From now on we assume that $M$ is orientable if $\mathbf{K}=\mathbf{R}$.

Let $\left(M, g_{\mathbf{K}}, \nabla\right)$ and $\left(M^{\prime}, g_{\mathbf{K}}^{\prime}, \nabla^{\prime}\right)$ be manifolds with $\mathbf{K}$-metrics and compatible connections, and let $\pi: M \times M^{\prime} \rightarrow M$ and $\pi^{\prime}: M \times M^{\prime} \rightarrow M^{\prime}$ be the projections. The canonical isomorphism

$$
\pi^{*} T M \oplus \pi^{*} T M^{\prime} \cong T\left(M \times M^{\prime}\right)
$$

defines a K-metric $\tilde{g}_{\mathbf{K}}=\pi^{*} g_{\mathbf{K}} \oplus \pi^{\prime *} g_{\mathbf{K}}^{\prime}$ and compatible connection $\tilde{\nabla}=\pi^{*} \nabla \oplus$ $\pi^{\prime *} \nabla^{\prime}$ in $T\left(M \times M^{\prime}\right)$. Extending the pointwise isomorphisms $\operatorname{Gr}\left(T_{m} M, g_{\mathbf{K} m}\right) \star$ $\operatorname{Gr}\left(T_{m^{\prime}} M^{\prime}, g_{\mathbf{K}_{m}^{\prime}}\right) \cong \operatorname{Gr}\left(T_{\left(m, m^{\prime}\right)} M \times M^{\prime}, \tilde{g}_{\mathbf{K}}\right)$ given by Theorem 4.2 , we get

5.2 Proposition. There is a unique group homomorphism

$$
\Phi: G r\left(M, g_{\mathbf{K}}\right) \star G r\left(M^{\prime}, g_{\mathbf{K}}^{\prime}\right) \rightarrow G r\left(M \times M^{\prime}, \tilde{g}_{\mathbf{K}}\right)
$$

such that the following diagram commutes:

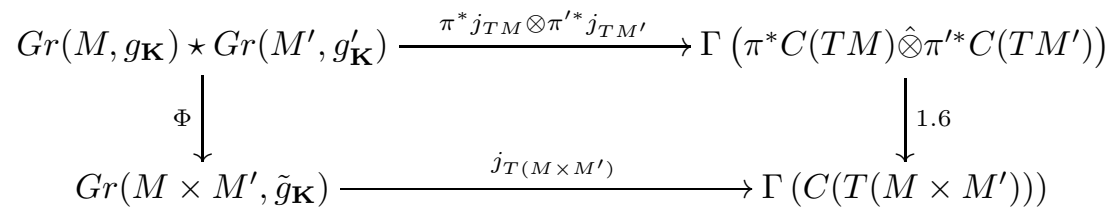

The map $F$ is compatible with gradings and covering projections and hence defines an isomorphism of $G D C$ s of $O_{\mathbf{K}}(1,1)$.

Proof. This is straightforward.

\section{$5.1 C(T)$ modules and quantization.}

5.3 Definition. Let $M$ be a manifold and $\nabla$ a connection in $T M$.

(a) A graded $C(T)$ module (over $(M, \nabla))$ is a graded real vector bundle $(E, \varepsilon)$ over $M$ with a graded connection $\nabla^{E}$, and a linear bundle map $R: C(T) \rightarrow E n d(E)$ such that:

(i) For all $m \in M,\left(E_{m}, \varepsilon_{m}, R_{m}\right)$ is a graded $C\left(T_{m}\right)$ module.

(ii) For $X \in \Gamma(T), c \in \Gamma(C(T))$ and $e \in \Gamma(E)$,

$$
\nabla_{X}^{E}(R(c)(e))=R\left(\nabla_{X} c\right)(e)+R(c)\left(\nabla_{X}^{E} e\right) .
$$

(b) Let $\sigma^{E}: \Gamma\left(\operatorname{Hom}\left(T^{*}, T \oplus T^{*}\right)\right) \rightarrow \Gamma\left(\operatorname{Hom}\left(T^{*}, \operatorname{End}(E)\right)\right)$ be the symbol map (cf. 1.10) and let $\operatorname{Diff}^{k}(E)$ be the space of $k$ th order differential operators in $E$. The quantization map $Q^{E}: \Gamma\left(\operatorname{Hom}\left(T^{*}, T \oplus T^{*}\right)\right) \rightarrow \operatorname{Diff}^{1}(E)$ is defined by

$$
Q^{E}(s)=\sum_{a=1}^{a=n} \sigma^{E}(s)_{\varepsilon^{a}} \circ \nabla_{e_{a}}^{E},
$$

where $s \in \Gamma\left(\operatorname{Hom}\left(T^{*}, T \oplus T^{*}\right)\right),\left\{E, \cdots, e_{n}\right\}$ is a local basis of $T$ and $\left\{\varepsilon^{1}, \cdots, \varepsilon^{n}\right\}$ is the dual (i.e., $\varepsilon^{a}\left(e_{b}\right)=\delta_{b}^{a}$ ) local basis of $T^{*}$. Note that the $Q^{E}(s)$ are odd operators in that $Q^{E}(s) \Gamma\left(E_{ \pm}\right) \subseteq \Gamma\left(E_{\mp}\right)$. 
5.3.1 Remark. If we regard $\sigma^{E}(s) \in \Gamma\left(\operatorname{Hom}\left(T^{*}, \operatorname{End}(E)\right)\right.$ as an element of $\Gamma\left(\operatorname{Hom}\left(T^{*} \otimes E, E\right)\right)$ via the natural identification $\operatorname{Hom}\left(T^{*}, \operatorname{End}(E)\right) \cong \operatorname{Hom}\left(T^{*} \otimes\right.$ $E, E)$ and $\nabla^{E}: \Gamma(E) \rightarrow \Gamma\left(T^{*} \otimes E\right)$ is the covariant derivative, we have $Q^{E}(s)=$ $\sigma^{E}(s) \circ \nabla^{E}$ and thus $Q^{E}(s)$ is globally well defined independently of any choices of local bases.

5.4 Proposition. If $\tilde{g} \in \Gamma\left(\operatorname{Pin}\left(T \oplus T^{*}\right)\right)$ is parallel and if $\pi(\tilde{g})=g$, then

$$
Q^{E}(g \circ s)=(-1)^{g} R(\tilde{g}) \circ Q^{E}(s) \circ R\left(\tilde{g}^{-1}\right) \quad \forall s \in \Gamma\left(\operatorname{Hom}\left(T^{*}, T \oplus T^{*}\right)\right) .
$$

In particular, if $M$ has a $\nabla$-parallel $\mathbf{K}$-metric, $\tilde{g} \in G r\left(M, g_{\mathbf{K}}\right)$ and $\pi(\tilde{g})=g \in$ $O\left(M, g_{\mathbf{K}}\right)^{\prime}$, then

$$
Q^{E}(g \circ s)=(-1)^{g} R(\tilde{g}) \circ Q^{E}(s) \circ R\left(\tilde{g}^{-1}\right) \quad \forall s \in I\left(M, g_{\mathbf{K}}\right) .
$$

Proof. We have

$$
\begin{aligned}
Q^{E}(g \circ s) & =\sum_{a=1}^{a=n} \sigma^{E}(g \circ s)_{\varepsilon^{a}} \circ \nabla_{e_{a}}^{E} \\
& =\sum_{a=1}^{a=n}(-1)^{g} R(\tilde{g}) \circ \sigma^{E}(s)_{\varepsilon^{a}} \circ R\left(\tilde{g}^{-1}\right) \circ \nabla_{e_{a}}^{E} \quad \text { (by 1.10) } \\
& =\sum_{a=1}^{a=n}(-1)^{g} R(\tilde{g}) \circ \sigma^{E}(s)_{\varepsilon^{a}} \circ \nabla_{e_{a}}^{E} \circ R\left(\tilde{g}^{-1}\right) \quad(\text { by } 5.3(\mathrm{ii})) \\
& =(-1)^{g} R(\tilde{g}) \circ Q^{E}(s) \circ R\left(\tilde{g}^{-1}\right) .
\end{aligned}
$$

The map $Q^{E}: I\left(M, g_{\mathbf{K}}\right) \rightarrow$ Diff $^{1}(E)$ extends to a unique algebra homomorphism $Q^{E}: T\left(I\left(M, g_{\mathbf{K}}\right)\right) \rightarrow \operatorname{Diff}(E)$ from the real tensor algebra of $I\left(M, g_{\mathbf{K}}\right)$ to the algebra of all differential operators in $E$ by the formula

$$
Q^{E}\left(h_{1} \otimes h_{2} \otimes \cdots \otimes h_{k}\right)=Q^{E}\left(h_{1}\right) \circ Q^{E}\left(h_{2}\right) \circ \cdots \circ Q^{E}\left(h_{k}\right) .
$$

By Proposition 5.4, the extended map satisfies

$$
Q^{E}(g(t))=(-1)^{g k} R(\tilde{g}) \circ Q^{E}(t) \circ R\left(\tilde{g}^{-1}\right)
$$

if

$$
t \in T^{k}\left(I\left(M, g_{\mathbf{K}}\right)\right), \tilde{g} \in G r\left(M, g_{\mathbf{K}}\right) \text { and } \pi(\tilde{g})=g .
$$

Hence, even $O\left(M, g_{\mathbf{K}}\right)^{\prime}$-invariant elements of $T\left(I\left(M, g_{\mathbf{K}}\right)\right)$ get mapped to $\operatorname{Gr}\left(M, g_{\mathbf{K}}\right)$-invariant differential operators in $\operatorname{Diff}(E)$. The eigenspaces of such an operator (if it has any) have a natural $G r\left(M, g_{\mathbf{K}}\right)$ action.

The basic $O\left(M, g_{\mathbf{K}}\right)^{\prime}$-invariant is the metric $\lambda_{I\left(M, g_{\mathbf{K}}\right)}$. Its dual $\lambda_{I\left(M, g_{\mathbf{K}}\right)}^{-1}$ is an invariant element of $S^{2}\left(I\left(M, g_{\mathbf{K}}\right)\right) \subset T\left(I\left(M, g_{\mathbf{K}}\right)\right)$ and one easily sees that $\lambda_{I\left(M, g_{\mathbf{K}}\right)}^{-1}$ equals

$$
\begin{gathered}
2\left(\tau_{g}^{-1} \otimes I d_{T^{*}}+I d_{T^{*}} \otimes \tau_{g}^{-1}\right) \quad \text { if } \mathbf{K}=\mathbf{R}, \\
2\left(\tau_{g}^{-1} \otimes I d_{T^{*}}+I d_{T^{*}} \otimes \tau_{g}^{-1}+\tau_{g}^{-1} \circ \rho^{*}(i)\right. \\
\left.\otimes \rho^{*}(i)+\rho^{*}(i) \otimes \tau_{g}^{-1} \circ \rho^{*}(i)\right) \quad \text { if } \mathbf{K}=\mathbf{C}
\end{gathered}
$$


and

$$
\begin{gathered}
2\left(\tau_{g}^{-1} \otimes I d_{T^{*}}+I d_{T^{*}} \otimes \tau_{g}^{-1}+\tau_{g}^{-1} \circ \rho^{*}(i) \otimes \rho^{*}(i)+\rho^{*}(i) \otimes \tau_{g}^{-1} \circ \rho^{*}(i)+\tau_{g}^{-1} \circ \rho^{*}(j)\right. \\
\otimes \rho^{*}(j)+\rho^{*}(j) \otimes \tau_{g}^{-1} \circ \rho^{*}(j)+\tau_{g}^{-1} \circ \rho^{*}(k) \\
\left.\otimes \rho^{*}(k)+\rho^{*}(k) \otimes \tau_{g}^{-1} \circ \rho^{*}(k)\right) \quad \text { if } \mathbf{K}=\mathbf{H} .
\end{gathered}
$$

This implies the first part of the

5.5 Corollary. (i) The differential operator $Q^{E}\left(\lambda_{I\left(M, g_{\mathrm{K}}\right)}^{-1}\right)$ commutes with the action of $\operatorname{Gr}\left(M, g_{\mathbf{K}}\right)$ on $\Gamma(E)$.

(ii) The principal symbol of $Q^{E}\left(\lambda_{I\left(M, g_{\mathbf{K}}\right)}^{-1}\right)$ at $\alpha \in T^{*}$ is $2 \operatorname{dim}_{\mathbf{R}} \mathbf{K} g^{-1}(\alpha, \alpha) I d_{E}$.

Proof. Let us first prove (ii) when $\mathbf{K}=\mathbf{R}$. The principal symbol of $Q^{E}\left(\lambda_{I\left(M, g_{\mathbf{K}}\right)}^{-1}\right)$ at $\alpha \in T^{*}$ is

$$
2 \sigma^{E}\left(\tau_{g}^{-1}\right)_{\alpha} \circ \sigma^{E}\left(I d_{T^{*}}\right)_{\alpha}+2 \sigma^{E}\left(I d_{T^{*}}\right)_{\alpha} \circ \sigma^{E}\left(\tau_{g}^{-1}\right)_{\alpha},
$$

which by definition is equal to

$$
2 R\left(\tau_{g}^{-1}(\alpha)\right) \circ R\left(I d_{T^{*}}(\alpha)\right)+2 R\left(I d_{T^{*}}(\alpha)\right) \circ R\left(\tau_{g}^{-1}(\alpha)\right) .
$$

Since $R$ defines a $C(T)$ module, this is

$$
4 B\left(\tau_{g}^{-1}(\alpha), I d_{T^{*}}(\alpha)\right) I d_{E}=4 B\left(\tau_{g}^{-1}(\alpha), \alpha\right) I d_{E},
$$

which reduces to $2 g^{-1}(\alpha, \alpha) I d_{E}$ using the definitions of $B$ and $\tau_{g}$ (cf. 3.4). This proves (ii) if $\mathbf{K}=\mathbf{R}$ and the proof of the other cases is similar; one uses $B\left(\tau_{g}^{-1}(\alpha), \alpha\right)$ $=B\left(\tau_{g}^{-1} \rho^{*}(i)(\alpha), \rho^{*}(i) \alpha\right)=B\left(\tau_{g}^{-1} \rho^{*}(j)(\alpha), \rho^{*}(j) \alpha\right)$, etc.

Now consider the restriction of $Q^{E}$ to $S^{2}\left(I\left(M, g_{\mathbf{K}}\right)\right)$, the real $\operatorname{dim}_{\mathbf{R}} \mathbf{K}\left(2 \operatorname{dim}_{\mathbf{R}} \mathbf{K}+\right.$ 1)-dimensional space of symmetric two tensors on $I\left(M, g_{\mathbf{K}}\right)$.

5.6 Theorem. Suppose there exists a nonzero $\lambda_{I\left(M, g_{\mathrm{K}}\right)}$-isotropic vector $z \in I(M$, $\left.g_{\mathbf{K}}\right)$ such that $Q^{E}(z \otimes z)=Q(z)^{2}=0$. Then:

(i) The image of $Q^{E}: S^{2}\left(I\left(M, g_{\mathbf{K}}\right)\right) \rightarrow \operatorname{Diff(}(E)$ is one-dimensional and spanned by $Q^{E}\left(\lambda_{I\left(M, g_{\mathbf{K}}\right)}^{-1}\right)$.

(ii) If $x, y \in I\left(M, g_{\mathbf{K}}\right)$, then

$$
Q^{E}(x) Q^{E}(y)+Q^{E}(y) Q^{E}(x)=\frac{1}{\operatorname{dim}_{\mathbf{R}} \mathbf{K}} \quad \lambda_{I\left(M, g_{\mathbf{K}}\right)}(x, y) \quad Q^{E}\left(\lambda_{I\left(M, g_{\mathbf{K}}\right)}^{-1}\right) .
$$

(iii) If $x \in I\left(M, g_{\mathbf{K}}\right)$, then $Q^{E}(x) \circ Q^{E}\left(\lambda_{I\left(M, g_{\mathbf{K}}\right)}^{-1}\right)=Q^{E}\left(\lambda_{I\left(M, g_{\mathbf{K}}\right)}^{-1}\right) \circ Q^{E}(x)$.

Proof. The map $Q^{E}: S^{2}\left(I\left(M, g_{\mathbf{K}}\right)\right) \rightarrow \operatorname{Diff}(E)$ is $O\left(M, g_{\mathbf{K}}\right)^{\prime}$-equivariant in the sense of Proposition 5.4 and the group $O\left(M, g_{\mathbf{K}}\right)^{\prime}$ acts transitively on the space of nonzero $\lambda_{I\left(M, g_{\mathbf{K}}\right)}$-isotropic vectors in $I\left(M, g_{\mathbf{K}}\right)$. Hence $Q^{E}(z)^{2}=0$ implies that $Q^{E}(x \otimes x)=Q^{E}(x)^{2}=0$ for all $\lambda_{I\left(M, g_{\mathbf{K}}\right)}$-isotropic vectors $x$ in $I\left(M, g_{\mathbf{K}}\right)$.

The kernel of $Q^{E}$ in $S^{2}\left(I\left(M, g_{\mathbf{K}}\right)\right)$ therefore contains all sums of squares of isotropic vectors. This latter space is of real codimension one in $S^{2}\left(I\left(M, g_{\mathbf{K}}\right)\right)$ and hence the real codimension of $\operatorname{Ker} Q^{E}$ is at most one. Since $Q^{E}\left(\lambda_{I\left(M, g_{\mathrm{K}}\right)}^{-1}\right)$ is nonzero by Corollary 5.5, $\operatorname{Ker} Q^{E}$ is of codimension exactly one and this proves part (i).

Now if we take $x, y \in I\left(M, g_{\mathbf{K}}\right)$, then $x \otimes y+y \otimes x \in S^{2}\left(I\left(M, g_{\mathbf{K}}\right)\right)$ and applying $Q$ we get

$$
Q^{E}(x) Q^{E}(y)+Q^{E}(y) Q^{E}(x)=f(x, y) Q^{E}\left(\lambda_{I\left(M, g_{\mathbf{K}}\right)}^{-1}\right)
$$


for some number $f(x, y)$ which clearly defines a bilinear form on $I\left(M, g_{\mathbf{K}}\right)$. Taking $g \in O\left(M, g_{\mathbf{K}}\right)^{\prime}$ and $\tilde{g} \in G r\left(M, g_{\mathbf{K}}\right)$ such that $\pi(\tilde{g})=g$ we have

$$
\begin{aligned}
f(g \circ x, g \circ y) Q^{E}\left(\lambda_{I\left(M, g_{\mathbf{K}}\right)}^{-1}\right)=Q^{E}(g \circ x) Q^{E}(g \circ y)+Q^{E}(g \circ y) Q^{E}(g \circ x) \\
\quad=R(\tilde{g}) \circ\left(Q^{E}(x) Q^{E}(y)+Q^{E}(y) Q^{E}(x)\right) \circ R(\tilde{g})^{-1} \quad(\text { by } 5.4) \\
\quad=f(x, y) R(\tilde{g}) \circ Q^{E}\left(\lambda_{I\left(M, g_{\mathbf{K}}\right)}^{-1}\right) \circ R(\tilde{g})^{-1} \\
\quad=f(x, y) Q^{E}\left(\lambda_{I\left(M, g_{\mathbf{K}}\right)}^{-1}\right),
\end{aligned}
$$

and hence $f(g \circ x, g \circ y)=f(x, y)$. Since an $O\left(M, g_{\mathbf{K}}\right)^{\prime}$-invariant symmetric bilinear form on $I\left(M, g_{\mathbf{K}}\right)$ is proportional to $\lambda_{I\left(M, g_{\mathbf{K}}\right)}$, this means that $f=k \lambda_{I\left(M, g_{\mathbf{K}}\right)}$. By the proof of Corollary 5.5,

$$
Q^{E}\left(\lambda_{I\left(M, g_{\mathbf{K}}\right)}^{-1}\right)=2 \operatorname{dim}_{\mathbf{R}} \mathbf{K}\left(Q^{E}\left(I d_{T^{*}}\right) Q^{E}\left(\tau_{g}^{-1}\right)+Q^{E}\left(\tau_{g}^{-1}\right) Q^{E}\left(I d_{T^{*}}\right)\right)
$$

and hence $f\left(I d_{T^{*}}, \tau_{g}^{-1}\right)=\frac{1}{2 \operatorname{dim}_{\mathbf{R}} \mathbf{K}}$. Since $\lambda_{I\left(M, g_{\mathrm{K}}\right)}\left(I d_{T^{*}}, \tau_{g}^{-1}\right)=\frac{1}{2}$ (by $3.4(\mathrm{ii})$ ), this proves (ii).

To prove (iii) let $x \in I\left(M, g_{\mathbf{K}}\right)$ be an isotropic vector and choose $y \in I\left(M, g_{\mathbf{K}}\right)$ such that $\lambda_{I\left(M, g_{\mathrm{K}}\right)}^{-1}(x, y) \neq 0$. Multiplying (ii) on the left and on the right by $Q^{E}(x)$ and using $Q^{E}(x)^{2}=0$ we see easily that $Q^{E}(x) \circ Q^{E}\left(\lambda_{I\left(M, g_{\mathbf{K}}\right)}^{-1}\right)=Q^{E}\left(\lambda_{I\left(M, g_{\mathrm{K}}\right)}^{-1}\right) \circ$ $Q^{E}(x)$. Every element of $I\left(M, g_{\mathbf{K}}\right)$ is a linear combination of isotropic vectors. So the same equation is valid for arbitrary $x$ in $I\left(M, g_{\mathbf{K}}\right)$.

5.6.1 Remark. As we shall see later, Theorem 5.4(ii) and Theorem 5.6 imply all classical Hodge type identities for Riemannian $(\mathbf{K}=\mathbf{R})$, Kähler $(\mathbf{K}=\mathbf{C})$ and hyper-Kähler $(\mathbf{K}=\mathbf{H})$ manifolds.

5.7 Definition. Let $M$ be a manifold and $\nabla$ a connection in $T M$. A graded $C(T)$ module $\left(E, \varepsilon, \rho, \nabla^{E}\right)$ is said to be flat if $Q^{E}\left(I d_{T^{*}}\right)^{2}=0$.

5.7.1 Proposition. Let $M$ be an oriented manifold and $\nabla$ a torsion free connection in TM. Let $\left(V, \varepsilon_{V}, \nabla^{V}\right)$ be a graded vector bundle with graded flat connection $\nabla^{V}$. Then $\left(\Lambda\left(T^{*}\right) \otimes V, \varepsilon_{\Lambda} \otimes \varepsilon_{V}, \rho_{\Lambda\left(T^{*}\right)} \otimes I d_{V}, \nabla^{\Lambda\left(T^{*}\right)} \otimes \nabla^{V}\right)$ is a flat $C(T)$ module.

Proof. By 1.10 and 5.3, $\sigma^{\Lambda\left(T^{*}\right) \otimes V}\left(I d_{T}^{*}\right): \Gamma\left(T^{*} \otimes \Lambda\left(T^{*}\right) \otimes V\right) \rightarrow \Gamma\left(\Lambda\left(T^{*}\right) \otimes V\right)$ and the differential operator $Q^{\Lambda\left(T^{*}\right) \otimes V}\left(I d_{T^{*}}\right)$ are given by $\sigma^{\Lambda\left(T^{*}\right) \otimes V}\left(I d_{T}^{*}\right)(\alpha \otimes \omega \otimes v)=$ $\alpha \wedge \omega \otimes v$ and

$$
Q^{\Lambda\left(T^{*}\right) \otimes V}\left(I d_{T^{*}}\right)(\omega \otimes v)=\sum_{a=1}^{a=n} \varepsilon_{a} \wedge\left(\nabla_{e_{a}}^{\Lambda\left(T^{*}\right)} \omega \otimes v+\omega \otimes \nabla_{e_{a}}^{V} v\right) .
$$

Hence $Q^{E}\left(I d_{T^{*}}\right)$ is the exterior covariant derivative operator since $\nabla$ is torsion free and $Q^{E}\left(I d_{T^{*}}\right)^{2}=0$ since $\nabla^{V}$ is flat.

5.7.2 Remark. One can show conversely that if $(E, \varepsilon, R)$ is a graded flat $C(T)$ module over $(M, \nabla)$, then $\nabla$ is torsion free and $(E, \varepsilon, R)$ is of the form given in 5.7.1 up to isomorphism.

By Proposition 5.1, for any orientable manifold $M$ with $\mathbf{K}$-metric $g_{\mathbf{K}}$ and compatible connection $\nabla,\left(I\left(M, g_{\mathbf{K}}\right), \lambda_{I\left(M, g_{\mathbf{K}}\right)}\right)$ is canonically isometric to $\left(\mathbf{K}^{2}, \lambda\right)$ and the group $O\left(M, g_{\mathbf{K}}\right)^{\prime}$ is canonically isomorphic to the matrix group $O_{\mathbf{K}}(1,1)$. Hence, roughly speaking, Theorem 5.4(ii) and Theorem 5.6 can be interpreted as defining a "representation" of the action of $\left.O_{\mathbf{K}}(1,1)\right)$ on $\left(\mathbf{K}^{2}, \lambda\right)$ in the space of smooth 
sections of $E$, a flat graded $C(T)$ module $E$ over $\left(M, g_{\mathbf{K}}, \nabla\right)$. Let us now make this idea more precise.

5.8 Definition. Let $\mathfrak{g}_{\mathbf{K}}$ be the real Lie superalgebra whose centre and even part is $\mathbf{R} \lambda^{-1}$ and whose odd part is $\mathbf{K}^{2}$ with bracket $[x, y]=\frac{1}{\operatorname{dim}_{\mathbf{R}} \mathbf{K}} \lambda(x, y) \lambda^{-1}$. The group $O_{\mathbf{K}}(1,1)$ acts naturally by automorphisms on $\mathfrak{g}_{\mathbf{K}}$. A $\mathbf{Z}_{2}$-projective representation of the pair $\left(O_{\mathbf{K}}(1,1), \mathfrak{g}_{\mathbf{K}}\right)$ in a graded vector space $(V, \varepsilon)$ is a $\mathbf{Z}_{2}$-projective representation $A$ of $O_{\mathbf{K}}(1,1)$ in $(V, \varepsilon)$ (cf. 2.5) together with a Lie superalgebra representation $B: \mathfrak{g}_{\mathbf{K}} \rightarrow \operatorname{End}(V)$ satisfying the compatibility condition

$$
B(g(s))=(-1)^{\operatorname{deg}(s) \operatorname{deg}(\widehat{A(g)})} \widehat{A(g)} \circ B(s) \circ \widehat{A(g)}^{-1} \quad \forall s \in \mathfrak{g}_{\mathbf{K}}, \forall g \in O_{\mathbf{K}}(1,1),
$$

where $\widehat{A(g)}$ is any element of $G L_{+}(V) \cup G L_{-}(V)$ lifting $A(g)$.

One can now interpret Theorem 5.4(ii) and Theorem 5.6 as defining a $\mathbf{Z}_{2^{-}}$ projective representation of an algebraic structure depending only on $\mathbf{K}$ and not on $\left(M, g_{\mathbf{K}}, \nabla\right)$ or $\left(E, \varepsilon, \rho, \nabla^{E}\right)$.

5.9 Theorem/Definition. Let $\left(M, g_{\mathbf{K}}, \nabla\right)$ be an orientable manifold with nondegenerate $\mathbf{K}$-metric $g_{\mathbf{K}}$ (of arbitrary signature) and Levi-Civita connection $\nabla$, and let $\left(E, \varepsilon, \rho, \nabla^{E}\right)$ be a flat graded $C(T)$ module. Let $[\rho]: O_{\mathbf{K}}(1,1) \rightarrow G L_{+}(\Gamma(E)) \cup$ $G L_{-}(\Gamma(E)) /\{ \pm I d\}$ be the homomorphism obtained by factoring $\rho: G r\left(M, g_{\mathbf{K}}\right) \rightarrow$ $G L(\Gamma(E))$ through the double covering map $\operatorname{Gr}\left(M, g_{\mathbf{K}}\right) \rightarrow O_{\mathbf{K}}(1,1)$. Let $Q^{E^{\prime}}$ : $\mathfrak{g}_{\mathbf{K}} \rightarrow \operatorname{End}(\Gamma(E))$ be defined by $Q^{E^{\prime}}\left(\lambda^{-1}\right)=Q^{E}\left(\lambda_{I\left(M, g_{\mathbf{K}}\right)}^{-1}\right)$ and $Q^{E^{\prime}}(s)=Q^{E} 。$ $i_{M}(s)$ if $s \in \mathbf{K}^{2}$.

Then $\left(\Gamma(E), \varepsilon,[\rho], Q^{E^{\prime}}\right)$ is a $\mathbf{Z}_{2}$-projective representation of $\left(O_{\mathbf{K}}(1,1), \mathfrak{g}_{\mathbf{K}}\right)$. This representation will be called the associated Hodge representation.

5.9.1 Remark. The vector space $\Gamma(E)$ is a nuclear Fréchet space for the topology given by uniform convergence of all derivatives on compact subsets in all local trivializations and the operators $Q^{E} \circ i_{M}(x)\left(x \in \mathbf{K}^{2}\right)$ and $\rho(g)\left(g \in G r\left(M, g_{\mathbf{K}}\right)\right)$ are continuous since they are differential operators. We will refer to nuclear Fréchet (NF) Hodge representations when we need to take this topology into account.

5.2 Multiplicative properties of Hodge representations. Let $\left(M, g_{\mathbf{K}}, \nabla\right)$ and $\left(M^{\prime}, g_{\mathbf{K}}^{\prime}, \nabla^{\prime}\right)$ be two manifolds with $\mathbf{K}$-metrics and compatible connections, let $\left(E, \varepsilon, \rho, \nabla^{E}\right)$ and $\left(E^{\prime}, \varepsilon^{\prime}, \rho^{\prime}, \nabla^{E^{\prime}}\right)$ be respectively graded $C(T)$ and $C\left(T^{\prime}\right)$ modules and let $\pi: M \times M^{\prime} \rightarrow M, \pi^{\prime}: M \times M^{\prime} \rightarrow M^{\prime}$ be the projections. Since $T\left(M \times M^{\prime}\right) \cong$ $\pi^{*} T M \oplus \pi^{\prime *} T M^{\prime}$, it is clear that $\left(\pi^{*} E \otimes \pi^{\prime *} E^{\prime}, \pi^{*} \varepsilon \otimes \pi^{\prime *} \varepsilon^{\prime}, \pi^{*} \rho \hat{\otimes} \pi^{\prime *} \rho^{\prime}, \pi^{*} \nabla^{E} \otimes\right.$ $\left.\pi^{\prime *} \nabla^{E^{\prime}}\right)$ is a $C\left(T\left(M \times M^{\prime}\right)\right)$ module over $\left(M \times M^{\prime}, \pi^{*} g_{\mathbf{K}} \oplus \pi^{\prime *} g_{\mathbf{K}}^{\prime}, \nabla \oplus \nabla^{\prime}\right)$. For brevity, we will denote this $C\left(T\left(M \times M^{\prime}\right)\right)$ module by $\left(\tilde{E}, \tilde{\varepsilon}, \tilde{\rho}, \nabla^{\tilde{E}}\right)$.

It is well known that the map $\Psi: \Gamma(E) \otimes_{\text {alg }} \Gamma\left(E^{\prime}\right) \rightarrow \Gamma\left(\pi^{*} E \otimes \pi^{\prime *} E^{\prime}\right)$ defined by

$$
\Psi\left(e \otimes e^{\prime}\right)=\pi^{*} e \otimes \pi^{\prime *} e^{\prime}
$$

has dense image, say $S$, and by completion extends to an isomorphism of nuclear Fréchet spaces $\bar{\Psi}: \Gamma(E) \bar{\otimes} \Gamma\left(E^{\prime}\right) \rightarrow \Gamma\left(\pi^{*} E \otimes \pi^{\prime *} E^{\prime}\right)$. By Theorem 5.2 and Corollary 4.5 , the restriction of $\Psi$ defines an isomorphism

$$
(\Gamma(E), \varepsilon, \rho) \otimes_{\text {alg }}\left(\Gamma\left(E^{\prime}\right), \varepsilon^{\prime}, \rho^{\prime}\right) \cong(S, \tilde{\varepsilon}, \tilde{\rho})
$$

of $\mathbf{Z}_{2}$-projective representations of $O_{\mathbf{K}}(1,1)$. 
5.10 Lemma. For $x \in \mathbf{K}^{2}, e \in \Gamma(E)$ and $e^{\prime} \in \Gamma\left(E^{\prime}\right)$,

$$
\begin{aligned}
Q^{\tilde{E}^{\prime}}(x)\left(\pi^{*} e \otimes{\pi^{\prime}}^{*} e^{\prime}\right) & =\pi^{*} Q^{E^{\prime}}(x)(e) \otimes \pi^{\prime *} e^{\prime}+(-1)^{e} \pi^{*} e \otimes \pi^{\prime *} Q^{E^{\prime \prime}}(x)\left(e^{\prime}\right), \\
Q^{\tilde{E}^{\prime}}(x)\left(\pi^{*} e \otimes \pi^{\prime *} e^{\prime}\right) & =\pi^{*} Q^{E^{\prime}}(x)^{2}(e) \otimes \pi^{\prime *} e^{\prime}+\pi^{*} e \otimes{\pi^{\prime}}^{*} Q^{E^{\prime \prime}}(x)^{2}\left(e^{\prime}\right) .
\end{aligned}
$$

Proof. The first equation is a direct calculation following from 1.10.1, the corresponding result for symbols, and Proposition 3.5. Squaring (5B), cross-terms cancel and we get the second equation.

If $\left(E, \varepsilon, \rho, \nabla^{E}\right)$ and $\left(E^{\prime}, \varepsilon^{\prime}, \rho^{\prime}, \nabla^{E^{\prime}}\right)$ are flat, then the $C\left(T\left(M \times M^{\prime}\right)\right)$ module $\left(\tilde{E}, \tilde{\varepsilon}, \tilde{\rho}, \nabla^{\tilde{E}}\right)$ is also flat since, by equation $(5 \mathrm{C}),{Q^{\tilde{E}^{\prime}}}^{2}=0$ on a dense subset of $\Gamma(\tilde{E})$. Moreover, equation (5B) then implies that $\Psi$ defines an isomorphism

$$
\left(\Gamma(E), \varepsilon, Q^{E^{\prime}}\right) \otimes_{\text {alg }}\left(\Gamma\left(E^{\prime}\right), \varepsilon^{\prime}, Q^{E^{\prime \prime}}\right) \cong\left(S, \tilde{\varepsilon}, Q^{\tilde{E}^{\prime}}\right)
$$

of representations of the Lie superalgebra $\mathfrak{g}_{\mathbf{K}}$. By completion:

5.11 Theorem. The map $\bar{\Psi}: \Gamma(E) \bar{\otimes} \Gamma\left(E^{\prime}\right) \rightarrow \Gamma\left(\pi^{*} E \otimes \pi^{\prime *} E^{\prime}\right)$ is an isomorphism of the NF-representations of $\left(O_{\mathbf{K}}(1,1), \mathfrak{g}_{\mathbf{K}}\right)$ :

$$
\bar{\Psi}:\left(\Gamma(E), \varepsilon,[\rho], Q^{E^{\prime}}\right) \bar{\otimes}\left(\Gamma\left(E^{\prime}\right), \varepsilon^{\prime},\left[\rho^{\prime}\right], Q^{E^{\prime \prime}}\right) \cong\left(\Gamma(\tilde{E}), \tilde{\varepsilon},[\tilde{\rho}], Q^{\tilde{E}^{\prime}}\right) .
$$

\section{EXPLicit FORMUlae FOR ElEMENTS OF $G r\left(T, g_{\mathbf{K}}\right)$}

Let $\left(T, g_{\mathbf{K}}\right)$ be a $\mathbf{K}$-metric space of real dimension $m \operatorname{dim}_{\mathbf{R}} \mathbf{K}$. Choose a $\mathbf{K}$-linear orthogonal (i.e., $\left.g\left(I_{i}, I_{j}\right)= \pm \delta_{i j}\right)$ basis $\left\{I_{1}, I_{2}, \cdots, I_{m}\right\}$, and let $\left\{E_{1}, E_{2}, \cdots, E_{m}\right\}$ be the $g$-dual basis (i.e., $\left.E_{i}=\tau_{g}\left(I_{i}\right)\right)$ of $T^{*}$. Then

$$
T=\bigoplus_{i=1}^{i=m} \rho(\mathbf{K}) I_{i}
$$

is an orthogonal decomposition into $\mathbf{K}$-metric spaces. Hence, by Theorem 4.5, the restriction of the concatenation isomorphism

$$
C\left(\rho(\mathbf{K}) I_{1}\right) \hat{\otimes} \cdots \hat{\otimes} C\left(\rho(\mathbf{K}) I_{m}\right) \cong C(T)
$$

defines an isomorphism of GDCs of $O_{\mathbf{K}}(1,1)$ :

$$
\operatorname{Gr}\left(\rho(\mathbf{K}) I_{1},\left.g_{\mathbf{K}}\right|_{\rho(\mathbf{K}) I_{1}}\right) \star \cdots \star G r\left(\rho(\mathbf{K}) I_{m},\left.g_{\mathbf{K}}\right|_{\rho(\mathbf{K}) I_{m}}\right) \cong G r\left(T, g_{\mathbf{K}}\right) .
$$

This isomorphism reduces the problem of finding explicit formulae for elements of $\operatorname{Gr}\left(T, g_{\mathbf{K}}\right)$ in the general case to the same problem in the one $\mathbf{K}$-dimensional case. In this chapter we will give a complete description of $\operatorname{Gr}\left(T, g_{\mathbf{K}}\right)$ when $\mathbf{K}=\mathbf{R}$ and $\mathbf{K}=\mathbf{C}$, and a partial description when $\mathbf{K}=\mathbf{H}$ (cf. Remark 6.4.3 below).

6.1 The explicit description of $G r\left(T, g_{\mathbf{R}}\right)$ in $C(T)$. Let $T$ be a one-dimensional $\mathbf{R}$-metric space and let $g$ be the metric. Recall that

$$
O(T, g)^{\prime}=\left\{\left(\begin{array}{cc}
\lambda I d_{T} & 0 \\
0 & \frac{1}{\lambda} I d_{T^{*}}
\end{array}\right): \quad \lambda \in \mathbf{R}^{*}\right\} \quad \cup \quad\left\{\left(\begin{array}{cc}
0 & \mu \tau_{g}^{-1} \\
\frac{1}{\mu} \tau_{g} & 0
\end{array}\right): \quad \mu \in \mathbf{R}^{*}\right\} .
$$

Let $I \in T$ be a unit vector (i.e., of length $\operatorname{sign}(g)= \pm 1$ ) and let $E=\tau_{g}(I) \in T^{*}$ be its $g$-dual. Considering $T \oplus T^{*}$ as a subset of the Clifford algebra $C(T)$ we have the relations

$$
E^{2}=I^{2}=0 \quad \text { and } \quad E I+I E=\operatorname{sign}(g) .
$$


If $\lambda \in \mathbf{R}^{*}$, the vectors $|\lambda|^{-\frac{1}{2}}(\lambda E-I)$ and $-E+I$ satisfy $\left(|\lambda|^{-\frac{1}{2}}(\lambda E-I)\right)^{2}=\operatorname{sign}(g)$ and $(-E+I)^{2}=-\operatorname{sign}(g)$, and hence are in $\operatorname{Pin}_{-}\left(T \oplus T^{*}\right)$. Their product $|\lambda|^{-\frac{1}{2}}(\lambda E I+I E)$ is therefore in $\operatorname{Pin}_{+}\left(T \oplus T^{*}\right)$.

6.1 Proposition. (a) The map $\chi_{T}: S O(1,1) \rightarrow$ Pin $_{+}\left(T \oplus T^{*}\right)$ given by

$$
\chi_{T}\left(\left(\begin{array}{cc}
\lambda & 0 \\
0 & \frac{1}{\lambda}
\end{array}\right)\right)=\operatorname{sign}(g)|\lambda|^{-\frac{1}{2}}(\lambda E I+I E)
$$

is a group homomorphism and satisfies

$$
\pi \circ \chi_{T}\left(\left(\begin{array}{cc}
\lambda & 0 \\
0 & \frac{1}{\lambda}
\end{array}\right)\right)=\left(\begin{array}{cc}
\frac{1}{\lambda} I d_{T} & 0 \\
0 & \lambda I d_{T^{*}}
\end{array}\right) \in O(T, g)^{\prime} .
$$

(b) $\sigma_{T}=-E+I$ satisfies

$$
\pi\left(\sigma_{T}\right)=\left(\begin{array}{cc}
0 & \tau_{g}^{-1} \\
\tau_{g} & 0
\end{array}\right) \in O(T, g)^{\prime} .
$$

(c) We have the following relations:

$$
\sigma_{T}^{2}=-\operatorname{sign}(g) \text { and } \sigma \chi_{T}\left(\begin{array}{cc}
\lambda & 0 \\
0 & \frac{1}{\lambda}
\end{array}\right) \sigma^{-1}=\frac{\lambda}{|\lambda|} \chi_{T}\left(\left(\begin{array}{cc}
\frac{1}{\lambda} & 0 \\
0 & \lambda
\end{array}\right)\right) .
$$

6.1.1 Corollary. The group $\operatorname{Gr}(T, g)$ is generated by -1 , the image of $\chi_{T}$ (even graded) and $\sigma_{T}$ (odd graded) subject to the relations $6.1(\mathrm{c})$.

Proof. These results all follow by direct calculation using the Clifford algebra relations (6B). We prove part (b) as an example. For this, one has to show that

$$
(-1)(-E+I) E(-E+I)^{-1}=I \text { and }(-1)(-E+I) I(-E+I)^{-1}=E
$$

and this is immediate.

Now let $(T, g)$ be a metric space of signature $(r, s)$ and let $\left\{I_{1}, \cdots, I_{r+s}\right\}$ be a $g$-orthogonal basis of $T$ such that $g\left(I_{i}, I_{j}\right)= \pm \delta_{i j}$ for $1 \leq i \leq r+s$. Let $\left\{E_{1}, E_{2}, \cdots, E_{n}\right\}$ be the $g$-dual basis of $T^{*}$. For $1 \leq i \leq n$ and $\lambda \in \mathbf{R}^{*}$ we set

$$
\chi_{T_{i}}\left(\left(\begin{array}{cc}
\lambda & 0 \\
0 & \frac{1}{\lambda}
\end{array}\right)\right)=\operatorname{sign}\left(g_{i}\right)|\lambda|^{-\frac{1}{2}}\left(\lambda E_{i} I_{i}+I_{i} E_{i}\right) \quad \text { and } \quad \sigma_{T_{i}}=-E_{i}+I_{i},
$$

where $T_{i}$ is the subspace of $T$ spanned by $E_{i}$ and $g_{i}=\left.g\right|_{T_{i}}$.

6.2 Theorem. (a) The map $\chi_{T}: S O(1,1) \rightarrow \operatorname{Pin}_{+}\left(T \oplus T^{*}, B\right)$ given by

$$
\chi_{T}=\chi_{T_{1}} \chi_{T_{2}} \cdots \chi_{T_{n}}
$$

is a group homomorphism and satisfies

$$
\pi \circ \chi_{T}\left(\left(\begin{array}{cc}
\lambda & 0 \\
0 & \frac{1}{\lambda}
\end{array}\right)\right)=\left(\begin{array}{cc}
\frac{1}{\lambda} I d_{T} & 0 \\
0 & \lambda I d_{T^{*}}
\end{array}\right) \in O(T, g)^{\prime} .
$$

(b) $\chi_{T}$ is independent of the choice of orthogonal basis $\left\{I_{1}, \cdots, I_{n}\right\}$ of $T$.

(c) $\sigma_{T}=\sigma_{T_{1}} \sigma_{T_{2}} \cdots \sigma_{T_{n}}$ is in $\operatorname{Pin}_{+}\left(T \oplus T^{*}\right)$ if $n$ is even, in Pin $\left(T \oplus T^{*}\right)$ if $n$ is odd and satisfies

$$
\pi\left(\sigma_{T}\right)=\left(\begin{array}{cc}
0 & \tau_{g}^{-1} \\
\tau_{g} & 0
\end{array}\right) \in O(T, g)^{\prime} .
$$

(d) $\sigma_{T}$ depends only on the orientation of the orthogonal basis $\left\{I_{1}, \cdots, I_{n}\right\}$. 
(e) We have the following relations:

$$
\sigma_{T}^{2}=(-1)^{\frac{1}{2}(r-s)(r-s+1)} \text { and } \sigma \chi_{T}\left(\left(\begin{array}{cc}
\lambda & 0 \\
0 & \frac{1}{\lambda}
\end{array}\right)\right) \sigma^{-1}=\left(\frac{\lambda}{|\lambda|}\right)^{n} \chi_{T}\left(\left(\begin{array}{cc}
\frac{1}{\lambda} & 0 \\
0 & \lambda
\end{array}\right)\right) .
$$

(f) Let $(T, g)$ and $\left(T^{\prime}, g^{\prime}\right)$ be metric spaces and let $\left(T \oplus T^{\prime}, g \oplus g^{\prime}\right)$ be the direct sum. Then under the isomorphism $C(T) \hat{\otimes} C\left(T^{\prime}\right) \cong C\left(T \oplus T^{\prime}\right)$ of Proposition 1.6, $\chi_{T}\left(\left(\begin{array}{cc}\lambda & 0 \\ 0 & \frac{1}{\lambda}\end{array}\right)\right) \otimes \chi_{T^{\prime}}\left(\left(\begin{array}{cc}\lambda & 0 \\ 0 & \frac{1}{\lambda}\end{array}\right)\right)$ is mapped to $\chi_{T \oplus T^{\prime}}\left(\left(\begin{array}{cc}\lambda & 0 \\ 0 & \frac{1}{\lambda}\end{array}\right)\right)$ and, if $T$ and $T^{\prime}$ are oriented, $\sigma_{T} \otimes \sigma_{T^{\prime}}$ is mapped to $\sigma_{T \oplus T^{*}}$ (where $T \oplus T^{*}$ is given the direct sum orientation).

Proof. Parts (a), (c), (e) and (f) all follow directly from the properties of the concatenation isomorphism (6A) and 6.1. We postpone the proofs of (b) and (d) until later (cf. Proposition 6.5).

6.2.1 Corollary. The group $G r(T, g)$ is generated by -1 (even graded), the image of $\chi_{T}$ (even graded) and $\sigma_{T}$ (odd graded iff $T$ is odd-dimensional) subject to the relations 6.2(e). By 6.2(e), the isomorphism class of $G r(T, g)$ depends only on $r-s \bmod 4$ and four distinct cases occur.

6.2 The explicit description of $G r\left(T, g_{\mathbf{C}}\right)$ in $C(T)$. Let $\left(T, g_{\mathbf{C}}\right)$ be a real, twodimensional $\mathbf{C}$-metric space, i.e., $g_{\mathbf{C}}=(g, \rho)$ where $g$ is a (necessarily definite) metric and $\rho: \mathbf{C} \rightarrow \operatorname{End}(T)$ is a real algebra homomorphism. The action $\rho$ is completely determined by $\rho(i)=J$, which is a $g$-isometric complex structure on $T$. Note that by Definition $3.2, \rho^{*}(i)=J^{t^{-1}} \in \operatorname{End}\left(T^{*}\right)$.

By Theorem 3.3 the map $c_{T}: O_{\mathbf{C}}(1,1) \rightarrow O\left(T, g_{\mathbf{C}}\right)^{\prime}$ given by

$$
c_{T}\left(\left(\begin{array}{ll}
a & b \\
c & d
\end{array}\right)\right)=\left(\begin{array}{cc}
\rho(a) & \rho(b) \tau_{g}^{-1} \\
\rho^{*}(c) \tau_{g} & \rho^{*}(d)
\end{array}\right)
$$

is a group isomorphism. The group $O_{\mathbf{C}}(1,1)$ is not simple: defining $r: U(1) \rightarrow$ $O_{\mathbf{C}}(1,1)$ and $s: S L(2, \mathbf{R}) \rightarrow O_{\mathbf{C}}(1,1)$ by

$$
r\left(e^{i \theta}\right)=\left(\begin{array}{cc}
e^{i \theta} & 0 \\
0 & e^{i \theta}
\end{array}\right) \quad \text { and } \quad s\left(\left(\begin{array}{ll}
a & b \\
c & d
\end{array}\right)\right)=\left(\begin{array}{cc}
a & i b \\
-i c & d
\end{array}\right),
$$

one checks that $p=r \times s: U(1) \times S L(2, \mathbf{R}) \rightarrow O_{\mathbf{C}}(1,1)$ is a double covering map. We now show that $\operatorname{Gr}\left(T, g_{\mathbf{C}}\right)$ as a GDC of $O_{\mathbf{C}}(1,1)$ is isomorphic to this double cover. (Recall that $\operatorname{Gr}\left(T, g_{\mathbf{C}}\right)$ is trivially graded by 4.1.1.)

If $I \in T$ is a unit vector, then $\left\{I, I^{\prime}=J(I)\right\}$ is an orthogonal basis of $T$. Let $\left\{E, E^{\prime}=J^{t^{-1}}(E)\right\}$ be the $g$-dual basis of $T^{*}$. Then the following relations hold in $C(T)$ :

$$
I E+E I=I^{\prime} E^{\prime}+E^{\prime} I^{\prime}=\operatorname{sign}(g) \quad \text { and } \quad x y+y x=0
$$

for all other choices of $x, y \in\left\{I, I^{\prime}, E, E^{\prime}\right\}$.

6.3 Theorem. (a) The maps $\tilde{R}: U(1) \rightarrow C(T)$ and $\tilde{S}: S L(2, \mathbf{R}) \rightarrow C(T)$ given by

$$
\begin{aligned}
& \tilde{R}\left(e^{i \theta}\right)=1+\operatorname{sign}(g) \sin \theta\left(E^{\prime} I-E I^{\prime}\right)+(\cos \theta-1)\left(E I^{\prime} E^{\prime} I+E^{\prime} I E I^{\prime}\right), \\
& \tilde{S}\left(\left(\begin{array}{ll}
a & b \\
c & d
\end{array}\right)\right)=1+(a-1) I^{\prime} I E E^{\prime}+\operatorname{sign}(g)\left(b I^{\prime} I+c E E^{\prime}\right)+(d-1) E E^{\prime} I^{\prime} I
\end{aligned}
$$

take values in Pin $\left(T \oplus T^{*}\right)$ and are group homomorphisms with commuting images. 
(b) $\tilde{R}(-1) \tilde{S}\left(\left(\begin{array}{cc}-1 & 0 \\ 0 & -1\end{array}\right)\right)=-1$.

(c) Setting $\tilde{c_{T}}=\tilde{R} \times \tilde{S}$, the following diagram commutes

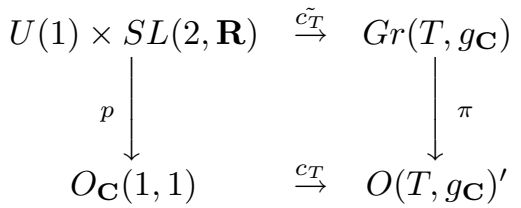

and $\tilde{c_{T}}$ is an isomorphism.

Proof. We set $\alpha=E^{\prime} I-E I^{\prime}, \beta=E I^{\prime} E^{\prime} I+E^{\prime} I E I^{\prime}, \varepsilon=E E^{\prime}$ and $\iota=I^{\prime} I$. Then the maps $\tilde{R}$ and $\tilde{S}$ can be written as

$$
\begin{gathered}
\tilde{R}\left(e^{i \theta}\right)=1+\operatorname{sign}(g) \sin \theta \alpha+(\cos \theta-1) \beta, \\
\tilde{S}\left(\left(\begin{array}{ll}
a & b \\
c & d
\end{array}\right)\right)=1+(a-1) \iota \varepsilon+\operatorname{sign}(g)(b \iota+c \varepsilon)+(d-1) \varepsilon \iota .
\end{gathered}
$$

It is easily checked that all products of elements in $\{\alpha, \beta\}$ with elements in $\{\varepsilon, \iota\}$ are 0 . Hence the images of $\tilde{R}$ and $\tilde{S}$ commute.

Using the Clifford algebra relations $(6 \mathrm{C})$, one verifies that

$$
\alpha^{T}=-\alpha, \alpha^{2}=-\beta, \alpha \beta=\beta \alpha=\alpha \quad \text { and } \quad \beta^{2}=\beta,
$$

that $\varepsilon^{T}=-\varepsilon, \iota^{T}=-\iota$ and that the following multiplication table holds:

\begin{tabular}{c|cccc} 
& $\varepsilon$ & $\iota$ & $\varepsilon \iota$ & $\iota \varepsilon$ \\
\hline$\varepsilon$ & 0 & $\varepsilon \iota$ & 0 & $\varepsilon$ \\
$\iota$ & $\iota \varepsilon$ & 0 & $\iota$ & 0 \\
$\varepsilon \iota$ & $\varepsilon$ & 0 & $\varepsilon \iota$ & 0 \\
$\iota \varepsilon$ & 0 & $\iota$ & 0 & $\iota \varepsilon$
\end{tabular}

From these relations it follows easily that

$$
\tilde{R}\left(e^{i \theta}\right)\left(\tilde{R}\left(e^{i \theta}\right)\right)^{T}=\tilde{S}\left(\left(\begin{array}{ll}
a & b \\
c & d
\end{array}\right)\right)\left(\tilde{S}\left(\left(\begin{array}{ll}
a & b \\
c & d
\end{array}\right)\right)\right)^{T}=1
$$

and hence that the images of $\tilde{R}$ and $\tilde{S}$ are in $\operatorname{Pin}\left(T \oplus T^{*}\right)$ (cf. 1.4), and, in fact, in $\operatorname{Pin}_{+}\left(T \oplus T^{*}\right)$ since they are connected and contain the identity. Similarly, one checks that $\tilde{R}$ and $\tilde{S}$ are group homomorphisms and this proves part (a). Part (b) is immediate.

To prove the first part of (c) one has to show that $\pi \circ \tilde{c_{T}}=c_{T} \circ p$, i.e.,

$$
\begin{aligned}
\pi \circ \tilde{c_{T}} & \left(e^{i \theta},\left(\begin{array}{ll}
a & b \\
c & d
\end{array}\right)\right) \\
& =\left(\begin{array}{cc}
\cos \theta I d_{T}+\sin \theta J & 0 \\
0 & \cos \theta I d_{T^{*}}+\sin \theta J^{t^{-1}}
\end{array}\right)\left(\begin{array}{cc}
a I d_{T} & b J \circ \tau_{g}^{-1} \\
-c J^{t^{-1}} \circ \tau_{g} & d I d_{T^{*}}
\end{array}\right) .
\end{aligned}
$$

For this, it is sufficient to prove the equality of both sides evaluated on the basis vectors $\left\{I, I^{\prime}, E, E^{\prime}\right\}$ of $T \oplus T^{*}$. As an example, consider the case of the vector $I$ 
and suppose $e^{i \theta}=1$ for simplicity. Evaluating the L.H.S. on $I$ we get

$$
\begin{aligned}
&(1+(a-1) \iota \varepsilon+\operatorname{sign}(g)(b \iota+c \varepsilon)+(d-1) \varepsilon \iota) I(1+(d-1) \iota \varepsilon \\
&-\operatorname{sign}(g)(b \iota+c \varepsilon)+(a-1) \varepsilon \iota) \\
&=(1+(a-1) \iota \varepsilon+\operatorname{sign}(g) c \varepsilon) I(1-\operatorname{sign}(g) c \varepsilon+(a-1) \varepsilon \iota),
\end{aligned}
$$

the equality following from $\iota I=I \iota=0$. Now, since $\varepsilon I \varepsilon=E E^{\prime} I E E^{\prime}=E E^{\prime 2} I E=0$, this reduces to

$$
I+(a-1)(\iota \varepsilon I+I \varepsilon \iota)+\operatorname{sign}(g) c(\varepsilon I-I \varepsilon) .
$$

But $\iota \varepsilon I+I \varepsilon \iota=I^{\prime} I E E^{\prime} I+I E E^{\prime} I^{\prime} I=I E\left(I^{\prime} E^{\prime}+E^{\prime} I^{\prime}\right) I=\operatorname{sign}(g) I E I=I$ and $\varepsilon I-I \varepsilon=E E^{\prime} I-I E E^{\prime}=-(E I+I E) E^{\prime}=-\operatorname{sign}(g) E^{\prime}$. Substituting in the above and simplifying this gives

$$
\pi \circ \tilde{c_{T}}\left(1,\left(\begin{array}{ll}
a & b \\
c & d
\end{array}\right)\right)(I)=a I-c E^{\prime}=a I d_{T}(I)-c J^{t-1} \circ \tau_{g}(I),
$$

which is the R.H.S. of (6D) evaluated on $I$ when $e^{i \theta}=1$. The general case is analogous.

The map $\tilde{c_{T}}$ is an isomorphism since $\pi \circ \tilde{c_{T}}$ is surjective and $-1 \in \operatorname{Im} \tilde{c_{T}}$.

6.3.1 Remark. One can extend $\tilde{R}: U(1) \rightarrow C(T)$ and $\tilde{S}: S L(2, \mathbf{R}) \rightarrow C(T)$ holomorphically to homomorphisms $\tilde{R}_{c}: \mathbf{C}^{*} \rightarrow C(T) \otimes \mathbf{C}$ and $\tilde{S}_{c}: S L(2, \mathbf{C}) \rightarrow$ $C(T) \otimes \mathbf{C}$. To define $\tilde{R}_{c}$ we set

$$
\tilde{R}_{c}(z)=1+\operatorname{sign}(g) \frac{1}{2 i}\left(z-\frac{1}{z}\right) \alpha+\left(\frac{1}{2}\left(z+\frac{1}{z}\right)-1\right) \beta \quad \forall z \in \mathbf{C}^{*},
$$

and to define $\tilde{S}_{c}$ we simply replace the real parameters in the formula for $\tilde{S}$ by complex parameters. The map $\tilde{c_{T c}}=\tilde{R}_{c} \times \tilde{S}_{c}: \mathbf{C}^{*} \times S L(2, \mathbf{C}) \rightarrow C(T) \otimes \mathbf{C}$ is then a holomorphic extension of $\tilde{c_{T}}$.

6.3.2 Corollary. Considering $\left(T, g_{\mathbf{C}}\right)$ as a two-dimensional $\mathbf{R}$-metric space, one can define the map $\chi_{T}: S O(1,1) \rightarrow \operatorname{Pin}_{+}\left(T \oplus T^{*}\right)$ and the element $\sigma_{T} \in P i n_{+}(T \oplus$ $\left.T^{*}\right)$ as in Theorem 6.2. Then

$$
\begin{aligned}
\chi_{T}\left(\left(\begin{array}{cc}
\lambda & 0 \\
0 & \frac{1}{\lambda}
\end{array}\right)\right) & =\tilde{c_{T}}\left(\frac{\lambda}{|\lambda|},\left(\begin{array}{cc}
\frac{1}{|\lambda|} & 0 \\
0 & |\lambda|
\end{array}\right)\right) \\
\sigma_{T} & =\operatorname{sign}(g) \tilde{c_{T}}\left(i,\left(\begin{array}{cc}
0 & -1 \\
1 & 0
\end{array}\right)\right) .
\end{aligned}
$$

Proof. Exercise.

Now let $\left(T, g_{\mathbf{C}}\right)$ be an arbitrary $\mathbf{C}$-metric space of signature $(2 r, 2 s)$ and let $\left\{I_{1}, I_{1}^{\prime}, \cdots, I_{r+s}, I_{r+s}^{\prime}\right\}$ be a $g$-orthogonal basis of $T$ such that $I_{i}^{\prime}=J\left(I_{i}\right)$ and $g\left(I_{i}, I_{i}\right)=g\left(I_{i}^{\prime}, I_{i}^{\prime}\right)= \pm 1$ for $1 \leq i \leq r+s$. Let $\left\{E_{1}, E_{1}^{\prime}, \cdots, E_{r+s}, E_{r+s}^{\prime}\right\}$ be the $g$-dual basis of $T^{*}$. The restriction of $g_{\mathbf{C}}$ to the real, two-dimensional subspace $T_{i}=\left\langle I_{i}, I_{i}^{\prime}\right\rangle$ of $T$ defines a $\mathbf{C}$-metric on $T_{i}$ and hence one can define the corresponding homomorphisms $c_{T_{i}}: U(1) \times S L(2, \mathbf{R}) \rightarrow C(T)$ by the formulae of Theorem 6.3 .

6.4 Theorem. (a) The map $\tilde{c_{T}}: U(1) \times S L(2, \mathbf{R}) \rightarrow C(T)$ given by

$$
\tilde{c_{T}}=\tilde{c}_{T_{1}}{\tilde{T_{2}}}_{2} \cdots \tilde{c_{T_{r+s}}}
$$


is a group homomorphism whose image is contained in $G r\left(T, g_{\mathbf{C}}\right)$ and the following diagram commutes:

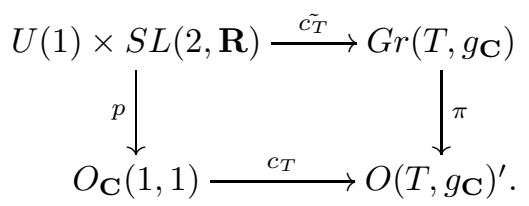

(b) The map $\tilde{c_{T}}$ is independent of the choice of g-orthogonal basis $\left\{I_{1}, I_{1}^{\prime}, \cdots\right.$, $\left.I_{r+s}, I_{r+s}^{\prime}\right\}$ of $T$.

(c) $\tilde{c_{T}}\left(-1,\left(\begin{array}{cc}-1 & 0 \\ 0 & -1\end{array}\right)\right)=(-1)^{r+s}$.

(d) If $r+s$ is odd, the map $\tilde{c_{T}}$ is an isomorphism of $U(1) \times S L(2, \mathbf{R})$ onto $\operatorname{Gr}\left(T, g_{\mathbf{C}}\right)$.

(e) If $r+s$ is even, the map $\tilde{c_{T}}$ factors through the projection $p: U(1) \times$ $S L(2, \mathbf{R}) \rightarrow O_{\mathbf{C}}(1,1)$ and trivializes the $G D C c_{T}^{-1} \circ \pi: G r\left(T, g_{\mathbf{C}}\right) \rightarrow O_{\mathbf{C}}(1,1)$.

(f) Let $\left(T, g_{\mathbf{C}}\right)$ and $\left(T^{\prime}, g_{\mathbf{C}}^{\prime}\right)$ be $\mathbf{C}$-metric spaces and let $\left(T \oplus T^{\prime}, g_{\mathbf{C}} \oplus g_{\mathbf{C}}^{\prime}\right)$ be the direct sum. Then under the isomorphism $C(T) \hat{\otimes} C\left(T^{\prime}\right) \cong C\left(T \oplus T^{\prime}\right)$ of Proposition 1.6, $\tilde{c_{T}}(x) \otimes \tilde{T_{T^{\prime}}}(x)$ is mapped to $c_{T \tilde{\oplus} T^{\prime}}(x)$ for all $x \in U(1) \times S L(2, \mathbf{R})$.

Proof. Everything except (b) follows directly from the properties of the concatenation isomorphism (6A) and Theorem 6.3. To prove (b) note that two homomorphisms $f, g: U(1) \times S L(2, \mathbf{R}) \rightarrow G r\left(T, g_{\mathbf{C}}\right)$ such that $\pi \circ f=\pi \circ g=c_{T} \circ p$ differ by a homomorphism $d: U(1) \times S L(2, \mathbf{R}) \rightarrow \mathbf{Z}_{2}$ which can only be the trivial homomorphism.

6.4.1 Corollary. Considering $\left(T, g_{\mathbf{C}}\right)$ as a $(2 r+2 s)$-dimensional $\mathbf{R}$-metric space, one can define the map $\chi_{T}: S O(1,1) \rightarrow \operatorname{Pin}_{+}\left(T \oplus T^{*}\right)$ and the element $\sigma_{T} \in$ $\operatorname{Pin}_{+}\left(T \oplus T^{*}\right)$ as in Theorem 6.2. Then

$$
\begin{aligned}
\chi_{T}\left(\left(\begin{array}{cc}
\lambda & 0 \\
0 & \frac{1}{\lambda}
\end{array}\right)\right) & =\tilde{c_{T}}\left(\frac{\lambda}{|\lambda|},\left(\begin{array}{cc}
\frac{1}{|\lambda|} & 0 \\
0 & |\lambda|
\end{array}\right)\right), \\
\sigma_{T} & =(-1)^{s} \tilde{c_{T}}\left(i,\left(\begin{array}{cc}
0 & -1 \\
1 & 0
\end{array}\right)\right) .
\end{aligned}
$$

Proof. This follows from 6.3.1.

We now give two formulae which will be useful later.

6.4.2 Proposition. If $\tilde{c_{T *}}: \mathbf{R} \oplus \operatorname{sl}(2, \mathbf{R}) \rightarrow C(T)$ is the derivative at the identity of $\tilde{c_{T}}$, then

$$
\tilde{c_{T *}}\left(\left(\begin{array}{ll}
0 & 1 \\
0 & 0
\end{array}\right)\right)=\sum_{i=1}^{i=r+s} \operatorname{sign}\left(g_{i}\right) I_{i}^{\prime} I_{i} \quad \text { and } \quad \tilde{c_{T}} *\left(\left(\begin{array}{ll}
0 & 0 \\
1 & 0
\end{array}\right)\right)=\sum_{i=1}^{i=r+s} \operatorname{sign}\left(g_{i}\right) E_{i} E_{i}^{\prime} \text {. }
$$

Proof. We prove only the first formula. Differentiating 6.4(a) at the identity gives

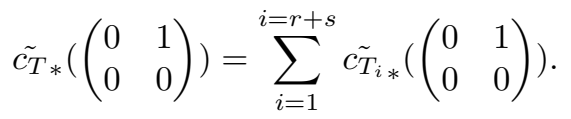

By definition,

$$
\tilde{c}_{T_{i *}}\left(\left(\begin{array}{ll}
0 & 1 \\
0 & 0
\end{array}\right)\right)=\left.\frac{d}{d t}\right|_{t=0} \tilde{c}_{T_{i}}\left(\exp \left(t\left(\begin{array}{cc}
0 & 1 \\
0 & 0
\end{array}\right)\right)\right)=\left.\frac{d}{d t}\right|_{t=0} \tilde{c}_{T_{i}}\left(\left(\begin{array}{ll}
1 & t \\
0 & 1
\end{array}\right)\right)
$$


and using the explicit formula of 6.3(a), this is clearly $\operatorname{sign}\left(g_{i}\right) I_{i}^{\prime} I_{i}$.

6.4.3 Remark. Let $\left(T, g_{\mathbf{H}}=(g, \rho)\right)$ be an $\mathbf{H}$-metric space. As is well known, the group $O_{\mathbf{H}}(1,1)(=S p(1,1))$ is isomorphic to the universal cover of $S O_{+}(4,1)$ and hence the GDC (trivially graded by 4.1.1) $\operatorname{Gr}\left(T, g_{\mathbf{H}}\right) \rightarrow O_{\mathbf{H}}(1,1)$ is necessarily (and canonically) isomorphic to the trivial double cover $O_{\mathbf{H}}(1,1) \times \mathbf{Z}_{2} \rightarrow O_{\mathbf{H}}(1,1)$. The author was not able to find a simple explicit formula for the canonical isomorphism $\Phi: O_{\mathbf{H}}(1,1) \times \mathbf{Z}_{2} \rightarrow G r\left(T, g_{\mathbf{H}}\right)$; but, using the results of section 6.2 , one can give explicit formulae for some of the elements of $\operatorname{Gr}\left(T, g_{\mathbf{H}}\right)$ as follows.

If $q \in \mathbf{H}$ is an imaginary unit quaternion, $\rho(q)$ is a $g$-isometric complex structure and hence defines a C-metric $g_{\mathbf{C}_{q}}$ on $T$ and a subgroup $\operatorname{Gr}\left(T, g_{\mathbf{C}_{q}}\right)$ of $\operatorname{Gr}\left(T, g_{\mathbf{H}}\right)$. The elements of $\operatorname{Gr}\left(T, g_{\mathbf{C}_{q}}\right)$ can then be described explicitly by Theorem 6.4. However, it is not true that every element of $\operatorname{Gr}\left(T, g_{\mathbf{H}}\right)$ is in a subgroup of this form.

At the Lie algebra level, $\Phi_{*}: o_{\mathbf{H}}(1,1) \rightarrow \operatorname{gr}\left(T, g_{\mathbf{H}}\right)$ is given by $\Phi_{*}=\pi_{*}{ }^{-1} \circ c_{T *}$ (cf. 4.1.3). The map $c_{T *}$ can be calculated by Theorem 3.3 and the formula for $\pi_{*}{ }^{-1}$ is well known so that one can calculate $\Phi_{*}$ explicitly. We will not do this, since the result will not be used in this paper. In [V], Verbitski gives an explicit isomorphism of $s o(4,1)$ with $\operatorname{gr}\left(T, g_{\mathbf{H}}\right)$.

6.3 The explicit description of $G r\left(T, g_{\mathbf{R}}\right)$ in the exterior algebra representation. As seen in Section 1, the map $\rho_{\Lambda\left(T^{*}\right)}: T \oplus T^{*} \rightarrow \operatorname{End}\left(\Lambda\left(T^{*}\right)\right)$ given by

$$
\begin{gathered}
\rho_{\Lambda\left(T^{*}\right)}(t)(\omega)=i_{t}(\omega) \quad \text { if } \quad t \in T, \\
\rho_{\Lambda\left(T^{*}\right)}(\alpha)(\omega)=e_{\alpha}(\omega) \quad \text { if } \quad \alpha \in T^{*},
\end{gathered}
$$

extends to an algebra isomorphism

$$
\rho_{\Lambda\left(T^{*}\right)}: C(T) \rightarrow \operatorname{End}\left(\Lambda\left(T^{*}\right)\right)
$$

and $\left(\Lambda\left(T^{*}\right), \varepsilon_{\Lambda\left(T^{*}\right)}, \rho_{\Lambda\left(T^{*}\right)}\right)$ is a graded $C(T)$ module. The next proposition identifies the generators of $\operatorname{Gr}\left(T, g_{\mathbf{R}}\right)$ in the exterior algebra representation in terms of more familiar operators. We write $1_{\Lambda}$ for the identity of the exterior algebra $\Lambda\left(T^{*}\right)$.

6.5 Proposition. (i) If $\omega \in \Lambda^{k}\left(T^{*}\right)$, then

$$
\rho_{\Lambda\left(T^{*}\right)} \circ \chi_{T}\left(\left(\begin{array}{cc}
\lambda & 0 \\
0 & \frac{1}{\lambda}
\end{array}\right)\right)(\omega)=\frac{\lambda^{k}}{|\lambda|^{\frac{n}{2}}}(\omega) .
$$

(ii) If $T$ is oriented, let $\left\{I_{1}, \cdots, I_{n}\right\}$ be a positively oriented orthonormal basis, let $\left\{E_{1}, \cdots, E_{n}\right\}$ be the $g$-dual basis, let $* \in \operatorname{End}\left(\Lambda\left(T^{*}\right)\right)$ be the Hodge star operator and let $\sigma_{T} \in C(T)$ be defined by $\sigma_{T}=\left(-E_{1}+I_{1}\right)\left(-E_{2}+I_{2}\right) \cdots\left(-E_{n}+I_{n}\right)$ as in Theorem 6.2(c). If $\omega \in \Lambda^{k}\left(T^{*}\right)$, then

$$
\rho_{\Lambda\left(T^{*}\right)}\left(\sigma_{T}\right)(\omega)=(-1)^{\left(n+\frac{k}{2}\right)(k-1)} * \omega .
$$

Proof. We set $e_{a}=\rho_{\Lambda\left(T^{*}\right)}\left(E_{a}\right)$ and $i_{a}=\rho_{\Lambda\left(T^{*}\right)}\left(I_{a}\right)$. Then $E_{1} \wedge E_{2} \wedge \cdots \wedge E_{k}=$ $e_{1} e_{2} \cdots e_{k}\left(1_{\Lambda}\right)$ and it is sufficient to prove (i) and (ii) for $\omega=E_{1} \wedge \cdots \wedge E_{k}$.

By definition,

$$
\begin{aligned}
& \rho_{\Lambda\left(T^{*}\right)} \circ \chi_{T}\left(\left(\begin{array}{cc}
\lambda & 0 \\
0 & \frac{1}{\lambda}
\end{array}\right)\right)\left(E_{1} \wedge \cdots \wedge E_{k}\right) \\
& \quad=(-1)^{s}|\lambda|^{-\frac{n}{2}}\left(\lambda e_{1} i_{1}+i_{1} e_{1}\right) \cdots\left(\lambda e_{n} i_{n}+i_{n} e_{n}\right) e_{1} e_{2} \cdots e_{k}\left(1_{\Lambda}\right) .
\end{aligned}
$$


A short calculation using the equations $i_{a}\left(1_{\Lambda}\right)=0, e_{a} e_{b}+e_{b} e_{a}=i_{a} i_{b}+i_{b} i_{a}=0$ and $e_{a} i_{b}+i_{b} e_{a}=g\left(I_{a}, I_{b}\right) \delta_{a b}$ shows that

$$
i_{a} e_{a}\left(e_{1} \cdots e_{k}\left(1_{\Lambda}\right)\right)= \begin{cases}0 & \text { if } a \in\{1,2, \cdots, k\} \\ g\left(I_{a}, I_{a}\right) e_{1} \cdots e_{k}\left(1_{\Lambda}\right) & \text { if } a \notin\{1,2, \cdots, k\}\end{cases}
$$

and that

$$
e_{a} i_{a}\left(e_{1} \cdots e_{k}\left(1_{\Lambda}\right)\right)= \begin{cases}g\left(I_{a}, I_{a}\right) e_{1} \cdots e_{k}\left(1_{\Lambda}\right) & \text { if } a \in\{1,2, \cdots, k\} \\ 0 & \text { if } a \notin\{1,2, \cdots, k\}\end{cases}
$$

Hence the above expression becomes

$$
\begin{aligned}
& =(-1)^{s}|\lambda|^{-\frac{n}{2}}\left(\lambda e_{1} i_{1}+i_{1} e_{1}\right) \cdots\left(\lambda e_{k} i_{k}+i_{k} e_{k}\right) \prod_{a=k+1}^{a=n}\left(g\left(I_{a}, I_{a}\right)\right) e_{1} e_{2} \cdots e_{k}\left(1_{\Lambda}\right) \\
& =(-1)^{s}|\lambda|^{-\frac{n}{2}} \lambda^{k} \prod_{a=1}^{a=n}\left(g\left(I_{a}, I_{a}\right)\right) e_{1} e_{2} \cdots e_{k}\left(1_{\Lambda}\right) \\
& =|\lambda|^{-\frac{n}{2}} \lambda^{k} e_{1} e_{2} \cdots e_{k}(1)=|\lambda|^{-\frac{n}{2}} \lambda^{k}\left(E_{1} \wedge \cdots \wedge E_{k}\right) .
\end{aligned}
$$

This proves part (i).

To prove part (ii), recall first that the Hodge star $* \beta$ of a $k$-form $\beta$ is the unique $(n-k)$-form such that for all $\alpha \in \Lambda^{k}\left(T^{*}\right)$,

$$
\alpha \wedge * \beta=(\alpha, \beta)_{\Lambda^{k}\left(T^{*}\right)} E_{1} \wedge \cdots \wedge E_{n},
$$

where $(, \quad)_{\Lambda^{k}\left(T^{*}\right)}$ is the symmetric bilinear form on $\Lambda^{k}\left(T^{*}\right)$ given by

$$
\left(\alpha_{1} \wedge \cdots \wedge \alpha_{k}, \beta_{1} \wedge \cdots \wedge \beta_{k}\right)_{\Lambda^{k}\left(T^{*}\right)}=\operatorname{det}\left[g\left(\alpha_{i}, \beta_{j}\right)\right] .
$$

It is easy to see that

$$
*\left(E_{1} \wedge \cdots \wedge E_{k}\right)=\prod_{a=1}^{a=k}\left(g\left(I_{a}, I_{a}\right)\right) E_{k+1} \wedge \cdots \wedge E_{n} .
$$

On the other hand,

$$
\begin{aligned}
\rho_{\Lambda\left(T^{*}\right)} & (\sigma)\left(E_{1} \wedge \cdots \wedge E_{k}\right)=\left(-e_{1}+i_{1}\right) \cdots\left(-e_{n}+i_{n}\right) e_{1} \cdots e_{k}\left(1_{\Lambda}\right) \\
= & \left(-e_{1}+i_{1}\right) \cdots\left(-e_{k}+i_{k}\right)\left(-e_{k+1}\right)\left(-e_{k+2}\right) \cdots\left(-e_{n}\right) e_{1} \cdots e_{k}\left(1_{\Lambda}\right) \\
= & (-1)^{(n-k)(k+1)}\left(-e_{1}+i_{1}\right) \cdots\left(-e_{k}+i_{k}\right) e_{1} \cdots e_{n}\left(1_{\Lambda}\right) \\
= & (-1)^{(n-k)(k+1)} i_{1} \cdots i_{k} e_{1} \cdots e_{n}\left(1_{\Lambda}\right) \\
= & (-1)^{(n-k)(k+1)}(-1)^{\frac{1}{2} k(k-1)} i_{1} \cdots i_{k} e_{k} \cdots e_{1} e_{k+1} e_{k+2} \cdots e_{n}\left(1_{\Lambda}\right) \\
= & (-1)^{(n-k)(k+1)+\frac{1}{2} k(k-1)} \prod_{a=1}^{a=k}\left(g\left(I_{a}, I_{a}\right)\right) e_{k+1} e_{k+2} \cdots e_{n}\left(1_{\Lambda}\right) \\
= & (-1)^{\left(n+\frac{k}{2}\right)(k-1)} \prod_{a=1}^{a=k}\left(g\left(I_{a}, I_{a}\right)\right) e_{k+1} e_{k+2} \cdots e_{n}\left(1_{\Lambda}\right) .
\end{aligned}
$$

Comparing with the formula for $*\left(E_{1} \wedge \cdots \wedge E_{k}\right)$ above, this proves part (ii).

6.5.1 Remark. From the formula in 6.5(ii) it is easy to see that there does not exist $g \in \operatorname{Pin}\left(T \oplus T^{*}\right)$ such that $\rho_{\Lambda\left(T^{*}\right)}(g)=*$. 
6.5.2 Remark. If $(,)_{\Lambda\left(T^{*}\right)}$ denotes the orthogonal sum of the symmetric bilinear forms on the $\Lambda^{k}\left(T^{*}\right)$, we have

$$
\left(e_{a}(\omega), \eta\right)_{\Lambda\left(T^{*}\right)}=\left(\omega, i_{a}(\eta)\right)_{\Lambda\left(T^{*}\right)}
$$

for $\omega, \eta \in \Lambda\left(T^{*}\right)$ and $a=1,2, \cdots, n$. Thus, if $t \in T$, the operators $i_{t}$ and $e_{\tau_{g}(t)}$ are mutual adjoints with respect to the bilinear form $(,)_{\Lambda\left(T^{*}\right)}$.

6.4 The explicit description of $G r\left(T, g_{\mathbf{C}}\right)$ in the exterior algebra representation. Let $\left(T, g_{\mathbf{C}}\right)$ be a $\mathbf{C}$-metric space. In this section we identify the image of $\rho_{\Lambda\left(T^{*}\right)} \circ \tilde{c_{T}}: U(1) \times S L(2, \mathbf{R}) \rightarrow \operatorname{End}\left(\Lambda\left(T^{*}\right)\right)$ in terms of more familiar operators (where possible). We will show that the formulae defining $\rho_{\Lambda\left(T^{*}\right)} \circ \tilde{c_{T}}$ give an explicit parametrization of the connected Lie subgroup of $\operatorname{End}\left(\Lambda\left(T^{*}\right)\right)$ which integrates the classical Hodge-Lefschetz $s l(2, \mathbf{R})$. To ease notation we write $\tilde{c_{\Lambda}}$ for $\rho_{\Lambda\left(T^{*}\right)} \circ \tilde{c_{T}}$

6.6 Proposition. If $\alpha_{1}, \cdots, \alpha_{k} \in T^{*}$, then

$\tilde{c_{\Lambda}}\left(e^{i \theta}\right)\left(\alpha_{1} \wedge \cdots \wedge \alpha_{k}\right)=\left(\cos \theta \alpha_{1}+\sin \theta J^{t^{-1}}\left(\alpha_{1}\right)\right) \wedge \cdots \wedge\left(\cos \theta \alpha_{k}+\sin \theta J^{t^{-1}}\left(\alpha_{k}\right)\right)$; i.e., $\tilde{c_{\Lambda}}\left(e^{i \theta}\right)$ is the unique automorphism of the exterior algebra $\Lambda\left(T^{*}\right)$ extending $\cos \theta I d_{T^{*}}+\sin \theta J^{t^{-1}} \in G L\left(T^{*}\right)$. If $\omega \in \Lambda^{p, q}$, then $\tilde{c_{\Lambda}}\left(e^{i \theta}\right)(\omega)=e^{i \theta(q-p)} \omega$.

Proof. By Corollary 6.4(a),

$$
\tilde{c_{\Lambda}}\left(e^{i \theta}\right) \circ e_{\alpha} \circ \tilde{c_{\Lambda}}\left(e^{-i \theta}\right)=e_{\cos \theta \alpha+\sin \theta J^{t-1} \alpha}
$$

for all $\alpha$ in $T^{*}$. Hence, since $\alpha_{1} \wedge \cdots \wedge \alpha_{k}=e_{\alpha_{1}} \cdots e_{\alpha_{k}}\left(1_{\Lambda}\right)$, we have

$$
\begin{aligned}
\tilde{c_{\Lambda}}\left(e^{i \theta}\right)\left(\alpha_{1} \wedge \cdots \wedge \alpha_{k}\right) & =\tilde{c_{\Lambda}}\left(e^{i \theta}\right) e_{\alpha_{1}} \cdots e_{\alpha_{k}}\left(1_{\Lambda}\right) \\
& =\prod_{i=1}^{i=k}\left(\tilde{c_{\Lambda}}\left(e^{i \theta}\right) e_{\alpha_{i}} \tilde{c_{\Lambda}}\left(e^{-i \theta}\right)\right) \tilde{c_{\Lambda}}\left(e^{i \theta}\right)\left(1_{\Lambda}\right) \\
& =\prod_{i=1}^{i=k}\left(e_{\cos \theta \alpha_{i}+\sin \theta J^{t-1} \alpha_{i}}\right) \tilde{c_{\Lambda}}\left(e^{i \theta}\right)\left(1_{\Lambda}\right) .
\end{aligned}
$$

By 6.4(a), one has $\tilde{c_{\Lambda}}\left(e^{i \theta}\right)=\rho_{\Lambda\left(T^{*}\right)} \circ{\tilde{c_{1}}}_{1}\left(e^{i \theta}\right) \cdots \rho_{\Lambda\left(T^{*}\right)} \circ \tilde{c}_{T_{r+s}}\left(e^{i \theta}\right)$ and by $6.3(\mathrm{a})$,

$$
\begin{aligned}
\rho_{\Lambda\left(T^{*}\right)} \circ \tilde{c}_{T_{i}}\left(e^{i \theta}\right)= & 1+\operatorname{sign}\left(g_{i}\right) \sin \theta\left(e_{E_{i}^{\prime}} i_{I_{i}}-e_{E_{i}} i_{I_{i}^{\prime}}\right) \\
& +(\cos \theta-1)\left(e_{E_{i}} i_{I_{i}^{\prime}} e_{E_{i}^{\prime}} i_{I_{i}}+e_{E_{i}^{\prime}} i_{I_{i}} e_{E_{i}} i_{I_{i}^{\prime}}\right) .
\end{aligned}
$$

The last factor in each term of this expression (except the first) is of the form $i_{t}$ for some $t$ in $T$. Since $i_{t}\left(1_{\Lambda}\right)=0$, this shows that $\rho_{\Lambda\left(T^{*}\right)} \circ \tilde{c}_{T_{i}}\left(e^{i \theta}\right)\left(1_{\Lambda}\right)=1_{\Lambda}$. Hence $\tilde{c_{\Lambda}}\left(e^{i \theta}\right)\left(1_{\Lambda}\right)=1_{\Lambda}$ and the proposition is proved.

Most of the operators in the image of $S L(2, \mathbf{R})$ under $\tilde{c_{\Lambda}}$ do not appear in the literature, at least not to the author's knowledge. However, the image of $\operatorname{sl}(2, \mathbf{R})$ under the derivative of $\tilde{c_{\Lambda}}$ is the classical Hodge-Lefschetz $\operatorname{sl}(2, \mathbf{R})$ acting in the exterior algebra.

6.7 Proposition. Let $\Omega=\sum_{i=1}^{i=r+s} \operatorname{sign}\left(g_{i}\right) E_{i} \wedge E_{i}^{\prime}$ be the canonical symplectic form on $T$, let $L: \Lambda \rightarrow \Lambda$ be exterior multiplication by $\Omega$ and let $L^{*}: \Lambda \rightarrow \Lambda$ be the adjoint of $L$ with respect to the symmetric bilinear form $(,)_{\Lambda\left(T^{*}\right)}(c f .6 .5 .2)$. Then

$$
\tilde{c_{\Lambda} *}\left(\left(\begin{array}{ll}
0 & 0 \\
1 & 0
\end{array}\right)\right)=L \quad \text { and } \quad \tilde{c_{\Lambda} *}\left(\left(\begin{array}{ll}
0 & 1 \\
0 & 0
\end{array}\right)\right)=L^{*} .
$$


Proof. Clearly, $\tilde{c_{\Lambda *}}=\rho_{\Lambda\left(T^{*}\right)} \circ \tilde{c_{T *}}$. By 6.4.2,

$$
\begin{aligned}
\rho_{\Lambda\left(T^{*}\right)} \circ \tilde{c_{T *}}\left(\left(\begin{array}{ll}
0 & 0 \\
1 & 0
\end{array}\right)\right) & =\sum_{i=1}^{i=r+s} \operatorname{sign}\left(g_{i}\right) \rho_{\Lambda\left(T^{*}\right)}\left(E_{i} E_{i}^{\prime}\right) \\
& =\sum_{i=1}^{i=r+s} \operatorname{sign}\left(g_{i}\right) e_{E_{i}} e_{E_{i}^{\prime}},
\end{aligned}
$$

and this operator is exterior multiplication by $\Omega$.

The second identity follows immediately by taking the adjoint of the first with respect to $(,)_{\Lambda\left(T^{*}\right)}$, and then applying 6.5.2 and 6.4.2.

\subsection{Proposition.}

$$
\tilde{c_{\Lambda}}\left(1,\left(\begin{array}{cc}
\frac{1}{x} & 0 \\
0 & x
\end{array}\right)\right)(\omega)=\frac{x^{k}}{x^{r+s}} \omega \quad \forall \omega \in \Lambda^{k}\left(T^{*}\right), \forall x \in \mathbf{R}^{*} .
$$

Proof. If $x>0, \tilde{c_{\Lambda}}\left(1,\left(\begin{array}{cc}\frac{1}{x} & 0 \\ 0 & x\end{array}\right)\right)=\chi_{\Lambda}\left(\left(\begin{array}{ll}x & 0 \\ 0 & \frac{1}{x}\end{array}\right)\right)$ by 6.3 .1 , and the result follows from 6.5 .

If $x<0$, then

$$
\begin{aligned}
\tilde{c_{\Lambda}}\left(1,\left(\begin{array}{cc}
\frac{1}{x} & 0 \\
0 & x
\end{array}\right)\right)(\omega) & =\tilde{c_{\Lambda}}\left(-1,\left(\begin{array}{ll}
1 & 0 \\
0 & 1
\end{array}\right)\right) \circ \tilde{c_{\Lambda}}\left(-1,\left(\begin{array}{cc}
-1 & 0 \\
0 & -1
\end{array}\right)\right) \circ \tilde{c_{\Lambda}}\left(1,\left(\begin{array}{cc}
\frac{1}{|x|} & 0 \\
0 & |x|
\end{array}\right)\right)(\omega) \\
& =(-1)^{k}(-1)^{r+s} \frac{|x|^{k}}{|x|^{r+s}} \omega
\end{aligned}
$$

by Proposition 6.6, Theorem 6.4(b) and the first part.

\section{The Hodge Representation of $\left(O_{\mathbf{R}}(1,1), \mathfrak{g}_{\mathbf{R}}\right)$ FOR MANIFOLDS WITH METRIC}

Let $(M, g, \nabla)$ be an oriented manifold with metric $g$ of signature $(r, s)$, and associated Levi-Civita connection $\nabla$. In this section we calculate explicitly the Hodge representation of $\left(O_{\mathbf{R}}(1,1), \mathfrak{g}_{\mathbf{R}}\right)$ associated to the $C(T)$ module $\left(\Lambda\left(T^{*}\right), \varepsilon_{\Lambda}, \rho_{\Lambda\left(T^{*}\right)}, \nabla^{\Lambda}\right)$. Recall that, by 5.7.1, this $C(T)$ module is flat and $Q\left(I d_{T^{*}}\right)=d$ is the exterior derivative.

The first ingredient in the Hodge representation is the $\mathbf{Z}_{2}$-projective action of $O_{\mathbf{R}}(1,1)$ obtained by projectivizing the action of $G r(M, g)$. By Propositions 6.2 and 6.5, the action of $\operatorname{Gr}(M, g)$ in $\Gamma\left(\Lambda\left(T^{*}\right)\right)$ is generated by the operators $\chi_{M, \lambda}$ $(\lambda \in \mathbf{R})$ and $\Sigma_{M}$ where if $\omega \in \Lambda^{k}\left(T^{*}\right)$,

$$
\chi_{M, \lambda}(\omega)=\left(\frac{\lambda^{k}}{|\lambda|^{\frac{n}{2}}}\right)(\omega) \quad \text { and } \quad \Sigma_{M}(\omega)=(-1)^{\left(n+\frac{k}{2}\right)(k-1)} * \omega .
$$

These operators satisfy the relations

$$
\Sigma_{M} \circ \chi_{M, \lambda} \circ \Sigma_{M}^{-1}=\left(\frac{\lambda}{|\lambda|}\right)^{n} \chi_{M, \frac{1}{\lambda}} \quad \text { and } \quad \Sigma_{M}^{2}=(-1)^{\frac{1}{2}(r-s)(r-s+1)} .
$$

The second ingredient in the Hodge representation is the quantization map $Q$ : $I(M, g) \rightarrow \operatorname{Diff}^{1}(\Lambda)$. The symbol map

$$
\sigma^{\Lambda}: I(M, g) \rightarrow \Gamma\left(\operatorname{Hom}\left(T^{*} \otimes \Lambda\left(T^{*}\right), \Lambda\left(T^{*}\right)\right)\right)
$$


is easily seen to be

$$
\sigma^{\Lambda}\left(\tau_{g}^{-1}\right)(\alpha \otimes \omega)=i_{\tau_{g}^{-1}(\alpha)} \omega \quad \text { and } \quad \sigma^{\Lambda}\left(I d_{T^{*}}\right)(\alpha \otimes \omega)=\alpha \wedge \omega
$$

By 6.5.2, the adjoint (with respect to the bilinear form on $\Lambda\left(T^{*}\right)$ ) of exterior multiplication by $\alpha$ is $i_{\tau_{g}^{-1}(\alpha)}$ and since $\nabla$ is the Levi-Civita connection, we have $Q\left(\tau_{g}^{-1}\right)=-Q\left(I d_{T^{*}}\right)^{*}=-d^{*}$, where $d^{*}$ is the formal adjoint (with respect to the integrated bilinear form) of the exterior derivative. Hence:

7.1 Proposition. The maps $Q: I(M, g) \rightarrow \operatorname{Diff}^{1}(\Lambda)$ and $Q^{\prime}: \mathfrak{g}_{\mathbf{R}} \rightarrow \operatorname{Diff}^{1}(\Lambda)$ are given by

$$
\left.Q\left(I d_{T^{*}}\right)=d, \quad Q\left(\tau_{g}^{-1}\right)=-d^{*} ; \quad Q^{\prime}\left(\left(\begin{array}{l}
0 \\
1
\end{array}\right)\right)=d, \quad Q^{\prime}\left(\begin{array}{l}
1 \\
0
\end{array}\right)\right)=-d^{*},
$$

and $Q^{\prime}\left(\lambda^{-1}\right)=-2 \square$, where $\square=d d^{*}+d^{*} d$ is the Hodge d'Alembertian.

Equations 5.6(ii) and 5.4(ii) can now be written as

(a) $Q^{\prime}(x) Q^{\prime}(y)+Q^{\prime}(y) Q^{\prime}(x)=-2 \lambda(x, y) \square \forall x, y \in \mathbf{R}^{2}$;

(b) $Q^{\prime}(g(x))=(-1)^{\tilde{g}} \rho_{\Lambda}(\tilde{g}) \circ Q^{\prime}(x) \circ \rho_{\Lambda}\left(\tilde{g}^{-1}\right) \forall x \in \mathbf{R}^{2}, \forall(g, \tilde{g}) \in O_{\mathbf{R}}(1,1)$

$\times \operatorname{Gr}(M, g)$ s.t $\pi(\tilde{g})=g$.

The equations (a) describe all anticommutators of the differential operators $d$ and $d^{*}$, i.e., $d^{2}=d^{* 2}=0$ and $\square=d d^{*}+d^{*} d$. The equations (b) reduce to:

\subsubsection{Proposition.}

$$
\begin{aligned}
& \text { (i) } \quad \lambda d=\chi_{M, \lambda} \circ d \circ \chi_{M, \lambda}^{-1} ; \quad \text { (ii) } \quad-d^{*}=(-1)^{n} \Sigma_{M} \circ d \circ \Sigma_{M}^{-1} \text {; } \\
& \text { (iii) } \frac{1}{\lambda} d^{*}=\chi_{M, \lambda} \circ d^{*} \circ \chi_{M, \lambda}^{-1} ; \quad(i v) \quad-d=(-1)^{n} \Sigma_{M} \circ d^{*} \circ \Sigma_{M}^{-1} \text {. }
\end{aligned}
$$

Proof. We prove (ii) as an example. If $x=\left(\begin{array}{l}0 \\ 1\end{array}\right)$ and $\tilde{g}=\sigma_{M}$, then $g=\left(\begin{array}{ll}0 & 1 \\ 1 & 0\end{array}\right)$ (by Proposition 6.2), $(-1)^{g}=(-1)^{n}$ (by Proposition 6.2) and $\rho_{\Lambda}\left(\sigma_{M}\right)=\Sigma_{M}$. Hence $Q^{\prime}(x)=Q\left(i_{M}((g(x)))=-d^{*}\right.$, and by substituting in (b) we get

$$
-d^{*}=(-1)^{n} \Sigma_{M} \circ d \circ \Sigma_{M}^{-1} \text {. }
$$

Rewriting the identities of 7.1.1 in terms of the degree operator and the Hodge star operator, we obtain the classical Hodge identities in signature $(r, s)$ :

7.1.2 Corollary. Let $\partial: \Gamma\left(\Lambda\left(T^{*}\right)\right) \rightarrow \Gamma\left(\Lambda\left(T^{*}\right)\right)$ be the degree operator $\partial(\omega)=$ $(-1)^{k} \omega$ if $\omega \in \Gamma\left(\Lambda^{k}\left(T^{*}\right)\right)$, and let $*$ be the Hodge star operator. Then, if $\omega \in$ $\Gamma\left(\Lambda^{k}\left(T^{*}\right)\right)$, we have

$$
\begin{aligned}
& \text { (i) } \quad[\partial, d]=d ; \quad(i i) \quad d^{*}(\omega)=(-1)^{1+r+(r+s) k} * \circ d \circ *(\omega) ; \\
& (\text { iii }) \quad\left[\partial, d^{*}\right]=-d^{*} ; \quad(i v) \quad d(\omega)=(-1)^{r+(r+s) k} * \circ d^{*} \circ *(\omega) .
\end{aligned}
$$

Proof. We leave the details to the reader.

If the metric $g$ is positive definite and $M$ is compact, $\square$ is elliptic and the natural injection Ker $\square \rightarrow H(M)$ is an isomorphism onto the de Rham cohomology $H(M)$. Since $\square$ commutes with the action of $G r(M, g)$ on $\Gamma\left(\Lambda\left(T^{*}\right)\right)$, this defines an action of $\operatorname{Gr}(M, g)$ on $H(M)$. The next proposition calculates the characters of 
this representation in terms of invariants of $M$. We write $\operatorname{Ker}^{k}(\square)$ for the harmonic forms of degree $k$.

7.2 Proposition. (i) If $b_{k}=\operatorname{dim} H^{k}(M)$ denotes the $k$ th Betti number of $M$, then

$$
\left.\operatorname{Tr} \chi_{M, \lambda}\right|_{K e r} \square=\sum_{k=0}^{k=n}\left(\frac{\lambda^{k}}{|\lambda|^{\frac{n}{2}}}\right) b_{k} .
$$

(ii) $\left.\operatorname{Tr} \Sigma_{M}\right|_{K e r} \square= \begin{cases}0 & \text { if } n \neq 0(\bmod 4), \\ (-1)^{\frac{n}{4}} \operatorname{sign}(M) & \text { if } n=0(\bmod 4) .\end{cases}$

Proof. Part (i) is immediate since $b_{k}=\operatorname{dim} \operatorname{Ker}^{k}(\square)$ by classical Hodge theory.

To prove part (ii) note first that if $n=1$ or $n=2(\bmod 4)$, then $\Sigma_{M}^{2}=-1$ and hence $\left.\operatorname{Tr} \Sigma_{M}\right|_{K e r} \square=0$. If $n=3(\bmod 4)$, then $\chi_{-1} \circ \Sigma_{M} \circ \chi_{-1}^{-1}=-\Sigma_{M}$ and hence $\left.\operatorname{Tr} \Sigma_{M}\right|_{\text {Ker }} \square=0$.

If $n=0(\bmod 4)$, then, since $\Sigma_{M}$ maps $k$-forms to $(n-k)$-forms and $\Sigma_{M}^{2}=1$, we have

$$
\left.\operatorname{Tr} \Sigma_{M}\right|_{K e r} \square=\left.\operatorname{Tr} \Sigma_{M}\right|_{K e r^{\frac{n}{2}} \square} \cdot
$$

But $\Sigma_{M}=(-1)^{\left(n+\frac{n}{4}\right)\left(\frac{n}{2}-1\right)} *=(-1)^{\frac{n}{4}} *$ on $\frac{n}{2}$-forms and so

$$
\left.\operatorname{Tr} \Sigma_{M}\right|_{\operatorname{Ker} \frac{n}{2}}=\left.(-1)^{\frac{n}{4}} \operatorname{Tr} *\right|_{\operatorname{Ker} \frac{n}{2}} \square .
$$

As is well known, $\operatorname{sign}(M)=\left.\operatorname{Tr} *\right|_{\operatorname{Ker}^{\frac{n}{2}}}$ (see, for example, chapter $\mathrm{V}$ in $[\mathrm{P}]$ ), and part (ii) is proved.

7.2.1 Remark. Restricting the isomorphism of Theorem 5.11 to kernels of Laplacians we get

$$
H^{*}(M) \otimes H^{*}\left(M^{\prime}\right) \cong H^{*}\left(M \times M^{\prime}\right)
$$

as graded representations of GDCs of $O_{\mathbf{R}}(1,1)$. Under this isomorphism, by $6.2(\mathrm{f})$,

$$
\chi_{M, \lambda} \hat{\otimes} \chi_{M^{\prime}, \lambda}=\chi_{M \times M^{\prime}, \lambda} \quad \text { and } \quad \Sigma_{M} \hat{\otimes} \Sigma_{M^{\prime}}=\Sigma_{M \times M^{\prime}},
$$

and taking traces, we recover the well-known results that the Euler characteristic and signature are multiplicative for products of compact oriented manifolds.

\section{The Hodge Representation of $\left(O_{\mathbf{C}}(1,1), \mathfrak{g}_{\mathbf{C}}\right)$ FOR MANIFOLDS OF KÄHLER TYPE}

Let $\left(M, g_{\mathbf{C}}\right)$ be a manifold with non-degenerate $\mathbf{C}$-metric $g_{\mathbf{C}}$ (of arbitrary signature) and suppose that the Levi-Civita connection is compatible with $g_{\mathbf{C}}$. In other words, $M$ is a manifold with non-degenerate metric $g$ and parallel (for the Levi-Civita connection) isometric almost complex structure $J$. In this section we calculate explicitly the Hodge representation of $\left(O_{\mathbf{C}}(1,1), \mathfrak{g}_{\mathbf{C}}\right)$ associated to the flat $C(T)$ module $\left(\Gamma\left(\Lambda\left(T^{*}\right)\right), \varepsilon_{\Lambda}, \rho_{\Lambda\left(T^{*}\right)}, \nabla^{\Lambda}\right)$.

The first ingredient of the Hodge representation is the $\mathbf{Z}_{\mathbf{2}}$-projective action of $O_{\mathbf{C}}(1,1)$ obtained by projectivizing the action of $\operatorname{Gr}\left(M, g_{\mathbf{C}}\right)$ in $\Gamma\left(\Lambda\left(T^{*}\right)\right)$. Although $\operatorname{Gr}\left(M, g_{\mathbf{C}}\right)$ as a GDC of $O_{\mathbf{C}}(1,1)$ depends non-trivially on $\left(M, g_{\mathbf{C}}\right)$, one can nevertheless, using Theorem 6.4, deprojectivize these representations to linear representations of a fixed GDC of $O_{\mathbf{C}}(1,1)$ in $\Gamma\left(\Lambda\left(T^{*}\right)\right)$. Consider the map $\tilde{c}_{M}: U(1) \times S L(2, \mathbf{R}) \rightarrow \Gamma\left(G r\left(T, g_{\mathbf{C}}\right)\right)$ given by

$$
\tilde{c_{M}}(X)(m)=\tilde{c}_{\tilde{T}_{m}}(X) \quad \forall m \in M, \forall X \in U(1) \times S L(2, \mathbf{R}) .
$$


By standard holonomy considerations, this map takes values in the parallel sections of $\operatorname{Gr}\left(T, g_{\mathbf{C}}\right) \rightarrow M$ and hence defines a homomorphism $\tilde{c}_{M}: U(1) \times S L(2, \mathbf{R}) \rightarrow$ $\operatorname{Gr}\left(M, g_{\mathbf{C}}\right)$. By $6.4(\mathrm{~d})$ and 6.4(e), this is an isomorphism if the complex dimension of $M$ is odd and factors through $U(1) \times S L(2, \mathbf{R}) \rightarrow O_{\mathbf{C}}(1,1)$ to trivialize $\operatorname{Gr}\left(M, g_{\mathbf{C}}\right) \rightarrow O_{\mathbf{C}}(1,1)$ if the complex dimension of $M$ is even. By $6.4(\mathrm{f})$, this deprojectivization process is compatible with tensor products. We write $c_{M, \Lambda}$ for $\rho_{\Lambda\left(T^{*}\right)} \circ \tilde{c_{M}}$.

The second ingredient of the Hodge representation is the quantization map $Q$ : $I\left(M, g_{\mathbf{C}}\right) \rightarrow \operatorname{Diff}^{1}(\Lambda)$. In terms of the basis $I d_{T^{*}}, \tau_{g}^{-1}, J \circ \tau_{g}^{-1}, J^{t^{-1}}$ of $I\left(M, g_{\mathbf{C}}\right)$, we have already seen that

$$
Q\left(I d_{T^{*}}\right)=d \quad \text { and } \quad Q\left(\tau_{g}^{-1}\right)=-d^{*} .
$$

Recall (see [W2]) that A. Weil defines the operator $d^{c} \in \operatorname{Diff}^{1}(\Lambda)$ by

$$
d^{c}=C^{-1} \circ d \circ C,
$$

where $C: \Lambda \rightarrow \Lambda$ is the bundle map

$$
C=\sum_{a, b} i^{a-b} P_{a, b}
$$

and $P_{a, b}: \Lambda \rightarrow \Lambda^{a, b}$ is projection onto the forms of type $(a, b)$. According to 6.6,

$$
C^{-1}=c_{\tilde{M}, \Lambda}\left(e^{i \frac{\pi}{2}}\right)
$$

and hence $d^{c}=c_{\tilde{M}, \Lambda}\left(e^{i \frac{\pi}{2}}\right) \circ d \circ c_{\tilde{M}, \Lambda}\left(e^{-i \frac{\pi}{2}}\right)$. But by Theorem 6.4(a)

$$
\pi \circ \tilde{c}_{M}\left(e^{i \frac{\pi}{2}}\right)=c_{M} \circ p\left(e^{i \frac{\pi}{2}}\right)=\left(\begin{array}{cc}
J & 0 \\
0 & J^{t-1}
\end{array}\right),
$$

and hence by Proposition 5.4,

$$
d^{c}=Q\left(\left(\begin{array}{cc}
J & 0 \\
0 & J^{t^{-1}}
\end{array}\right) \circ I d_{T^{*}}\right)=Q\left(J^{t^{-1}}\right) .
$$

Similarly, one calculates $Q\left(J \circ \tau_{g}^{-1}\right)$ and this proves the first part of the next proposition.

8.1 Proposition. (i) The maps $Q: I\left(M, g_{\mathbf{C}}\right) \rightarrow \operatorname{Diff}^{1}(\Lambda)$ and $Q^{\prime}: \mathfrak{g}_{\mathbf{C}} \rightarrow \operatorname{Diff}^{\mathrm{l}}(\Lambda)$ are given by

$$
\begin{aligned}
& Q\left(I d_{T^{*}}\right)=d, \quad Q\left(\tau_{g}^{-1}\right)=-d^{*} ; \quad Q^{\prime}\left(\left(\begin{array}{l}
0 \\
1
\end{array}\right)\right)=d, \quad Q^{\prime}\left(\left(\begin{array}{l}
1 \\
0
\end{array}\right)\right)=-d^{*} ; \\
& \left.Q\left(J \circ \tau_{g}^{-1}\right)=-d^{c *}, \quad Q\left(J^{t^{-1}}\right)=d^{c} ; \quad Q^{\prime}\left(\begin{array}{l}
i \\
0
\end{array}\right)\right)=-d^{c *}, \quad Q^{\prime}\left(\left(\begin{array}{c}
0 \\
i
\end{array}\right)\right)=d^{c},
\end{aligned}
$$

and $Q^{\prime}\left(\lambda^{-1}\right)=-4 \square$, where $\square=d d^{*}+d^{*} d$ is the Hodge d'Alembertian.

Proof. By substituting $x=I d_{T^{*}}$ and $y=\tau_{g}^{-1}$ in Theorem 6.4 we get

$$
-\square=\frac{1}{2} \lambda_{I\left(M, g_{\mathbf{C}}\right)}\left(I d_{T^{*}}, \tau_{g}^{-1}\right) Q\left(\lambda_{I\left(M, g_{\mathbf{C}}\right)}^{-1}\right),
$$

and $\left.\lambda_{I\left(M, g_{\mathbf{C}}\right)}\left(I d_{T^{*}}, \tau_{g}^{-1}\right)=\lambda\left(\left(\begin{array}{l}0 \\ 1\end{array}\right)\right),\left(\begin{array}{l}1 \\ 0\end{array}\right)\right)=\frac{1}{2}$ by $3.4(\mathrm{ii})$ and $5.1(\mathrm{ii})$. 
Equations 5.6(ii) and 5.4(ii) can now be written as

(a) $Q^{\prime}(x) Q^{\prime}(y)+Q^{\prime}(y) Q^{\prime}(x)=-2 \lambda(x, y) \square \forall x, y \in \mathbf{C}^{2}$.

(b) $Q^{\prime}(p(g)(z))=c_{\tilde{M}, \Lambda}(g) \circ Q^{\prime}(z) \circ c_{\tilde{M}, \Lambda}(g)^{-1} \forall z \in \mathbf{C}^{2}, \forall g \in U(1) \times S L(2, \mathbf{R})$. They imply all classical Hodge-Lefschetz identities in the following way.

Equation (a) describes all anticommutators of the differential operators $d, d^{*}, d^{c}$ and $d^{c *}$. For example, if $x=\left(\begin{array}{l}i \\ 0\end{array}\right), y=\left(\begin{array}{l}0 \\ i\end{array}\right)$, then $\lambda(x, y)=\frac{1}{2}, Q^{\prime}(x)=-d^{c *}$, $Q^{\prime}(y)=d^{c}$ and (a) is the classical identity

$$
d^{c} d^{c *}+d^{c *} d^{c}=\square .
$$

Equation (b) is an integrated version of the Hodge-Lefschetz identities of the form $-d^{c^{*}}=\left[L^{*}, d\right]$, etc. To see this, by differentiating (b) we get

$$
\text { (c) } Q^{\prime}\left(p_{*}(X)(z)\right)=\left[c_{M}, \Lambda_{*}(X), Q^{\prime}(z)\right] \forall X \in \mathbf{R} \oplus \operatorname{sl}(2, \mathbf{R}), \forall z \in \mathbf{C}^{2} \text {. }
$$

For example, if $z=\left(\begin{array}{l}0 \\ 1\end{array}\right)$ and $X=\left(\begin{array}{ll}0 & 1 \\ 0 & 0\end{array}\right)$, then $Q^{\prime}(z)=d, p_{*}(X)=\left(\begin{array}{ll}0 & i \\ 0 & 0\end{array}\right)$, $p_{*}(X)(z)=\left(\begin{array}{l}i \\ 0\end{array}\right), Q^{\prime}\left(p_{*}(X)(z)\right)=-d^{c *}$ and $c_{\tilde{M}, \Lambda_{*}}(X)=L^{*}$ (cf. 6.7) and hence (c) reduces to the classical identity

$$
-d^{c *}=\left[L^{*}, d\right] .
$$

8.1 A proof of the Hodge Index Theorem. Now suppose that $M$ is compact and that $g$ is positive definite. The action of $\operatorname{Gr}\left(M, g_{\mathbf{C}}\right)$ commutes with the Hodge Laplacian $\square$ and therefore acts on its kernel which we can identify with the cohomology $H^{*}(M)$ of $M$ by classical Hodge theory. The group homomorphism $c_{\tilde{M}, \Lambda}: U(1) \times S L(2, \mathbf{R}) \rightarrow \Gamma(\operatorname{End}(\Lambda))$ extends (cf. 6.3.1) to a group homomorphism $c_{\tilde{M}, \Lambda_{c}}: \mathbf{C}^{*} \times S L(2, \mathbf{C}) \rightarrow \Gamma(\operatorname{End}(\Lambda \otimes \mathbf{C}))$ whose image consists of parallel sections and commutes with $\square$. This gives a representation

$$
c_{\tilde{M}, \Lambda_{c}}: \mathbf{C}^{*} \times S L(2, \mathbf{C}) \rightarrow G L(K e r \square \otimes \mathbf{C}) .
$$

Recall from 6.4.1 that

$$
\Sigma_{M}=c_{M, \Lambda}\left(e^{i \frac{\pi}{2}},\left(\begin{array}{cc}
0 & -1 \\
1 & 0
\end{array}\right)\right)
$$

and from Proposition 7.2 that

$$
\left.\operatorname{Tr} \Sigma_{M}\right|_{\text {Ker }} \square=\left\{\begin{array}{lll}
0 & \text { if } n \neq 0 \quad(\bmod 4), \\
(-1)^{\frac{n}{4}} \operatorname{sign}(M) & \text { if } n=0 \quad(\bmod 4) .
\end{array}\right.
$$

8.2 Theorem (Hodge Index Theorem). Let $M$ be a compact Kähler manifold of real dimension $2 m$. Then

$$
\operatorname{sign}(M)=\sum_{p, q}(-1)^{p} h^{p, q}
$$

where $h^{p, q}=\operatorname{dim} H^{p, q}$.

Proof. The eigenvalues of $\left(\begin{array}{cc}0 & -1 \\ 1 & 0\end{array}\right)$ are $i$ and $-i$. Hence $\left(e^{i \frac{\pi}{2}},\left(\begin{array}{cc}0 & -1 \\ 1 & 0\end{array}\right)\right)$ and $\left(e^{i \frac{\pi}{2}},\left(\left(\begin{array}{cc}i & 0 \\ 0 & -i\end{array}\right)\right)\right.$ are conjugate in $\mathbf{C}^{*} \times S L(2, \mathbf{C})$ and have the same trace in a representation of $\mathbf{C}^{*} \times S L(2, \mathbf{C})$. 
By holomorphic extension of Proposition 6.8,

$$
c_{\tilde{M}, \Lambda_{c}}\left(1,\left(\begin{array}{cc}
i & 0 \\
0 & -i
\end{array}\right)\right)(\omega)=\frac{(-i)^{p+q}}{(-i)^{m}} \omega \quad \forall \omega \in \Lambda^{p, q}
$$

and hence by 6.6 ,

$$
c_{\tilde{M}, \Lambda_{c}}\left(e^{i \frac{\pi}{2}},\left(\begin{array}{cc}
i & 0 \\
0 & -i
\end{array}\right)\right)(\omega)=\frac{(-i)^{p+q}}{(-i)^{m}} e^{i \frac{\pi}{2}(q-p)} \omega=i^{m}(-1)^{p} \omega \quad \forall \omega \in \Lambda^{p, q} .
$$

Taking the trace on Ker $\square$ and using equation (8A) above we get

$$
\left.\operatorname{Tr} \Sigma_{M}\right|_{K e r} \square=i^{m} \sum_{p, q}(-1)^{p} h^{p, q} .
$$

The result now follows from equation (8B) above.

8.2 Multiplicative numerical invariants for compact Kähler and hyperKähler manifolds. For compact Kähler manifolds, restricting the isomorphism of Theorem 5.11 to kernels of Laplacians we get

$$
\operatorname{Ker} \square_{M} \otimes_{\mathbf{R}} \operatorname{Ker} \square_{M^{\prime}} \cong \operatorname{Ker} \square_{M \times M^{\prime}}
$$

as representations of $U(1) \times S L(2, \mathbf{R})$. Hence every element $x \in U(1) \times S L(2, \mathbf{R})$ gives rise to a (real) multiplicative invariant of compact Kähler manifolds $\left(M, g_{\mathbf{C}}\right) \mapsto$ $\left.\operatorname{Tr} c_{M, \Lambda}(x)\right|_{K e r} \square$ since $c_{M, \Lambda}(x) \hat{\otimes} c_{M^{\prime}, \Lambda}(x)=c_{M \times M^{\prime}, \Lambda}(x)$ (cf. 6.4(f)). Complexifying, every element $x \in \mathbf{C}^{*} \times S L(2, \mathbf{C})$ gives rise to a (complex) multiplicative invariant of compact Kähler manifolds. If $x$ is in a compact subgroup, this number is invariant under smooth deformations of the Kähler metric since the representation theory of compact groups is discrete. In general, $\left.\operatorname{Tr} c_{\tilde{M}, \Lambda}(g)\right|_{K e r} \square$ will not be a simple combination of the Hodge numbers $h^{p, q}$.

If $\mathbf{K}=\mathbf{H}$ and $\left(M, g_{\mathbf{H}}\right)$ is a compact hyper-Kähler manifold, by 6.4.3 there is a canonical isomorphism $\Phi_{M}: S p(1,1) \times \mathbf{Z}_{2} \rightarrow G r\left(M, g_{\mathbf{H}}\right)$ commuting with covering maps. Hence the $\mathbf{Z}_{2}$-projective representation of $S p(1,1)$ in $\Gamma\left(\Lambda\left(T^{*}\right)\right)$ can be deprojectivized to a linear representation of $S p(1,1)$ in $\Gamma\left(\Lambda\left(T^{*}\right)\right)$. Furthermore, this deprojectivization process is compatible with tensor products since $\Phi_{M}$ is unique. Restricting the isomorphism of Theorem 5.11 to kernels of Laplacians we get

$$
\operatorname{Ker} \square_{M} \otimes_{\mathbf{R}} \operatorname{Ker} \square_{M^{\prime}} \cong \operatorname{Ker} \square_{M \times M^{\prime}}
$$

as representations of $S p(1,1)$. Hence every element of $S p(1,1)$ (or complexifying, of $\operatorname{Spin}(5, \mathbf{C})$ ) gives rise to a multiplicative invariant of compact hyper-Kähler manifolds.

\section{ACKNOWLEDGMENTS}

The author thanks D. Bennequin and R. J. Stanton for many useful conversations.

\section{REFERENCES}

[ABS] Atiyah, M. F., Bott, R., and Shapiro, A. - Clifford Modules, Topology, vol. 3, Suppl. 1, 1963, 3-38. MR 29:5250

[B] Bernstein, J. - Lectures on SUSY (notes by P. Deligne and J. Morgan) in 'Quantum Fields and Strings: a course for mathematicians', Vol 1 (ed P. Deligne et al) - American Mathematical Society, Providence, RI, 1999.

[BdR] Bidal, P. and de Rham, G. - Les formes différentielles harmoniques, Comm. Math. Helvetica, vol. 19, 1946, 1-49. MR 8:93b 
[FKS] Figueroa-O'Farrill, J., Köhl, C. and Spence, B. - Supersymmetry and the cohomology of (hyper)Kähler manifolds, Nuclear Phys. B, vol 503(3), 1997, pp. 614-626. MR 98k:53062

[H] Hodge, W.V.D. - The theory and application of harmonic integrals. - Cambridge University Press, 1941. MR 2:296d MR 90g:58001 (reprint)

[Ho] Howe, R. - Dual pairs in physics: harmonic oscillators, photons, electrons and singletons, in 'Applications of group theory in physics and mathematical physics' (Chicago, 1982), Lectures in Appl. Math, 21. - Amer. Math. Soc., Providence, R.I., 1985, pp. 179-207 . MR 86i:22036

[K] Kodaira, K. - Über die Harmonischen Tensorfelder in Riemannschen Mannigfaltigkeiten I, Proc. Imp. Acad Tokyo, vol. 20, 1944, pp. 186-198. MR 7:329b

[P] Palais, R. S. (ed.) - Seminar on the Atiyah-Singer index theorem. - Annals of Math. Studies, 57, Princeton University Press, 1965. MR 33:6649

[S] Slupinski, M.J. - Dual Pairs in Pin $(p, q)$ and Howe Correspondances for the Spin Representation, Journal of Algebra, vol. 202, 1998, pp. 512-540. MR 99c:20065

[Sch] Scharlau, W. - Quadratic and hermitian forms. - Grundlehren der Math. Wissen., 270, Springer-Verlag, Berlin, 1985. MR 86k:11022

[V] Verbitsky, M.S. - Action of the Lie algebra of SO(5) on the cohomology of hyper-Kähler manifolds, Functional Anal. Appl., vol. 24, 1991, pp. 229-230. MR 92a:53095

[W1] Weil, A. - Sur la théorie des formes différentielles attachées à une variété analytique complexe, Comm. Math. Helv., vol. 20, 1947, pp. 110-116. MR 9:65a

[W2] Weil, A. - Variétés Kählériennes. - Hermann, Paris, 1958.

[Wi] Witten, E. - Problems in 'Quantum fields and strings: a course for mathematicians', Vol. 1 (ed. P. Deligne et al). - American Mathematical Society, Providence, RI, 1999. MR 2001c:81002

Université de Louis Pasteur et CNRS (URA 01), 7 rue René Descartes, 67084 Strasbourg Cedex, France

E-mail address: slupins@math.u-strasbg.fr 